\title{
A sample of 669 ultra steep spectrum radio sources to find high redshift radio galaxies
}

\author{
C. De Breuck ${ }^{1,2}$, W. van Breugel ${ }^{2}$, H.J.A. Röttgering ${ }^{1}$, and G. Miley ${ }^{1}$ \\ 1 Sterrewacht Leiden, Postbus 9513, 2300 RA Leiden, The Netherlands \\ e-mail: debreuck,miley,rottgeri@strw.leidenuniv.nl \\ ${ }^{2}$ Institute of Geophysics and Planetary Physics, Lawrence Livermore National Laboratory, L-413, Livermore, CA 94550, U.S.A. \\ e-mail: wil@igpp.ucllnl.org
}

Received November 4, 1999; accepted February 4, 2000

\begin{abstract}
Since radio sources with Ultra Steep Spectra (USS; $\alpha \lesssim-1.30 ; S \propto \nu^{\alpha}$ ) are efficient tracers of high redshift radio galaxies (HzRGs), we have defined three samples of such USS sources using the recently completed WENSS, TEXAS, MRC, NVSS and PMN radiosurveys. Our combined sample contains 669 sources with $S_{1400}>10 \mathrm{mJy}$ and covers virtually the entire sky outside the Galactic plane $\left(|b|>15^{\circ}\right)$. For our 2 largest samples, covering $\delta>-35^{\circ}$, we selected only sources with angular sizes $\Theta<1^{\prime}$. For 410 sources, we present radio-maps with $0^{\prime \prime} 3$ to $\sim 5^{\prime \prime}$ resolution from VLA and ATCA observations or from the FIRST survey, which allows the optical identification of these radio sources.

Comparison with spectrally unbiased samples at similar flux density levels, shows that our spectral index, flux density, and angular size selections do not affect the angular size distribution of the sample, but do avoid significant contributions by faint foreground spiral galaxies. We find that the spectral index distribution of 143000 sources from the WENSS and NVSS consists of a steep spectrum galaxy and a flat spectrum quasar population, with the relative contribution of flat spectrum sources doubling from $S_{1400}>0.1$ Jy to $S_{1400}>2.5 \mathrm{Jy}$. The identification fraction of our USS sources on the POSS $(R \lesssim 20)$ is as low as $15 \%$, independent of spectral index $\alpha<-1.30$. We further show that $85 \%$ of the USS sources that can be identified with an X-ray source are probably contained in galaxy clusters, and that $\alpha<-1.6$ sources are excellent Galactic pulsar candidates, because the percentage of these sources is four times higher in the Galactic plane.

Our sample has been constructed to start an intensive campaign to obtain a large sample of high redshift objects
\end{abstract}

$\overline{\text { Send offprint requests to: }}$ C. De Breuck $(z>3)$ that is selected in a way that does not suffer from dust extinction or any other optical bias ${ }^{1}$.

Key words: surveys - galaxies: active — radio continuum: galaxies

\section{Introduction}

Radio galaxies have now been found out to redshifts of $z=5.19$ (van Breugel et al. 1999b) and radio-loud quasars out to $z=4.72$ (Hook \& McMahon 1998). Although new optical selection techniques such as color-dropouts, deep spectroscopy of blank fields, and narrow-band Ly $\alpha$ imaging have now found galaxies at similar (Steidel et al. 1999) and even higher redshifts (up to $z \sim 5.75$; Dey et al. 1998; Weymann et al. 1998; Spinrad et al. 1998, Hu et al. 1999), radio sources are still the only objects that can be selected uniformly over all redshift ranges, and in a way that does not suffer from optical biases such as dust extinction, which is known to be important at these high redshifts (e.g. Hughes et al. 1997; Ivison et al. 1998; Dickinson 1998).

At low to moderate redshift $(z \lesssim 1)$, powerful radio sources are uniquely identified with massive ellipticals (Lilly \& Longair 1984; Owen \& Laing 1989; Best et al. 1998; McLure \& Dunlop 2000). The strongest indications that this is also true at higher redshifts comes from the near-IR Hubble $K-z$ diagram of radio galaxies which shows a remarkably close correlation from the

\footnotetext{
1 Appendices B, C and $\mathrm{D}$ are available in electronic form only, at http://www.edpsciences.org
} 


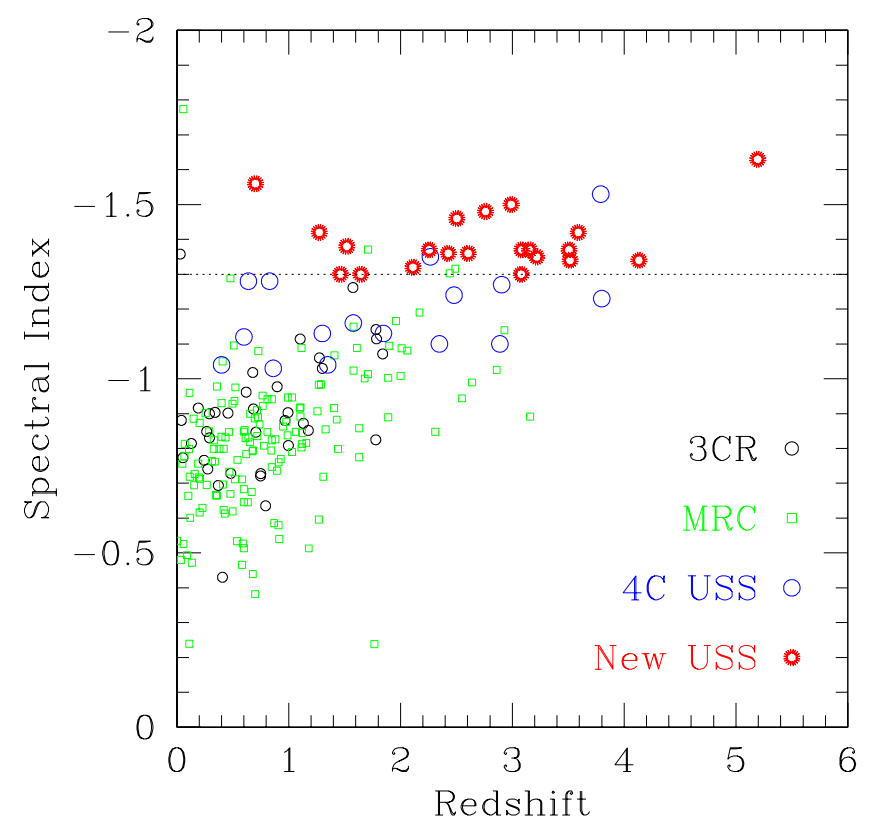

Fig. 1. $\alpha_{1400}^{325}$ against $z$ for 2 samples without spectral index selection (3CR, Spinrad et al. 1985 and MRC, McCarthy et al. 1996), and 2 USS samples (4C, Chambers et al. 1996a, and our new $\mathrm{WN} / \mathrm{TN}$ samples, as defined in this paper). Note that the correlation is present in the spectrally unbiased $3 \mathrm{CR}$ and $\mathrm{MRC}$, and that the $4 \mathrm{C}$ and our new USS samples are finding three to five times more $z>2$ radio galaxies than the MRC. The horizontal dotted line indicates the $\alpha_{1400}^{325}<-1.3$ cutoff used in our USS sample

present out to $z=5.19$ (Lilly 1989; Eales et al. 1997; van Breugel et al. 1998; van Breugel et al. 1999b). This suggests that we can use radio galaxies to study the formation and evolution of the most massive galaxies, which, by their implied star-formation history, can put important constraints on galaxy formation models, and even on cosmological parameters (e.g. Dunlop et al. 1996; Spinrad et al. 1997). Although the unification model for radio galaxies and quasars (e.g. Barthel 1989) suggests we could also use quasars as tracers, a detailed stellar population study of quasar host galaxies is almost impossible due to the extreme luminosity of the AGN. Furthermore, samples of radio sources designed to find large quantities of quasars require additional optical selections (e.g. Gregg et al. 1996; Hook \& McMahon 1998; White et al. 2000).

Considerable effort has been spent over the last decade to find these high redshift radio galaxies (HzRGs), which has lead to the discovery of more than 140 radio galaxies at redshifts $z>2$ (see e.g. De Breuck et al. 1998a for a recent summary). However by $z>3$, their numbers become increasingly sparse, and using flux limited radio surveys such as the 3CR $\left(S_{178}>10 \mathrm{Jy}\right.$; Laing et al. 1983), or the MRC strip $\left(S_{408}>0.95 \mathrm{Jy}\right.$; McCarthy et al. 1996), the highest redshift radio galaxy found so far is at $z \sim 3.2$ (Fig. 1; Rawlings et al. 1990; McCarthy et al. 1996). This redshift limit arises because radio power is correlated with redshift in bright flux limited samples, and an upper limit exists in the radio luminosity. Lowering the flux limit would not only substantially increase the number of sources in these samples, but at the same time the fraction of luminous very high redshift radio galaxies would decrease (Blundell et al. 1998; Jarvis et al. 1999). This fractional decrease would arise even if there is no decrease in co-moving space density at $z \gtrsim 2.5$. Such a redshift cutoff has been suggested by Bremer et al. (1998), but recently Jarvis et al. (1999) rule out a break at $z \lesssim 2.5$. To efficiently find large numbers of HzRGs in acceptable observing times, it is therefore necessary to apply additional selection criteria, at the expense of completeness.

By far the most successful selection criterion has been the ultra steep spectrum criterion (e.g. Röttgering et al. 1994; Chambers et al. 1996a; Blundell et al. 1998). Selecting sources with very steep radio spectra increases dramatically the chance of finding $z>2$ radio galaxies (Fig. 1). This technique is based on the results of Tielens et al. (1979) and Blumenthal \& Miley (1979), who found that the identification fraction on the POSS $(R \lesssim 20)$ decreases with steepening spectral index, consistent with the steeper sources being at higher redshifts. It is now getting clear that this correlation can be explained by a combination of a K-correction of a concave radio spectrum and an increasing spectral curvature with redshift (Krolik \& Chen 1991; Carilli et al. 1998; van Breugel et al. 1999a). To further investigate the $z-\alpha$ correlation, we have calculated spectral indices using the flux densities from the WENSS (Rengelink et al. 1997) and NVSS (Condon et al. 1998) catalogs for four different samples: the flux density limited 3CR (Spinrad et al. 1985) and MRC (McCarthy et al. 1996) surveys, and the USS samples from the $4 \mathrm{C}$ (Chambers et al. 1996a) and the one presented in this paper. The results (Fig. 1) show a trend for steeper spectral index sources to have higher redshifts in flux limited, spectrally unbiased samples, confirming the empirical relation out to the highest redshifts. The efficiency of the USS criterion is clearly illustrated by the fact that the 4C USS sample (Chambers et al. 1996a) contains $50 \% z>2$ sources, and by the early spectroscopic results on the USS samples presented in this paper, which indicate that $\sim 2 / 3$ of our sources have $z>2$. It is even more impressive to note that 13 of the 14 radio galaxies at $z>3.5$ we know of have been found from samples with a steep spectral index selection $^{2}$ ! The limitation of this technique is that the steepest spectrum sources are rare, comprising typically only $0.5 \%$ (at $\alpha<-1.30$ ) of a complete low frequency sample; therefore, large and deep all sky surveys are needed to obtain a significant sample of USS sources.

${ }^{2}$ The only exception is VLA J123642+6213 (Waddington et al. 1999), which has been identified in the HDF, but it does have a steep spectral index $\left(\alpha_{1400}^{8500}=-0.94\right)$. 
Table 1. Radio surveys

\begin{tabular}{|c|c|c|c|}
\hline & WENSS & TEXAS & MRC \\
\hline Frequency (MHz) & 325 & 365 & 408 \\
\hline Sky region $(\mathrm{J} 2000)$ & $\delta>+29^{\circ}$ & $-35.7<\delta<+71.5$ & $-85^{\circ}<\delta<+18.5$ \\
\hline \# of sources & 229576 & 67551 & 12141 \\
\hline Resolution & $54^{\prime \prime} \times 54^{\prime \prime} \operatorname{cosec} \delta$ & $10^{\prime \prime a}$ & $2^{\prime} .62 \times 2^{\prime} .86 \sec \left(\delta-35^{\circ} .5\right)$ \\
\hline $\begin{array}{l}\text { Position uncertainty } \\
\text { (strong sources) }\end{array}$ & $1^{\prime \prime} 5$ & $0^{\prime \prime} 5-1^{\prime \prime}$ & $8^{\prime \prime}$ \\
\hline RMS noise & $\sim 4 \mathrm{mJy}$ & $20 \mathrm{mJy}$ & $70 \mathrm{mJy}$ \\
\hline Flux density limit & $18 \mathrm{mJy}$ & $150 \mathrm{mJy}$ & $670 \mathrm{mJy}$ \\
\hline \multirow[t]{2}{*}{ Reference } & Rengelink et al. 1997 & Douglas et al. 1996 & Large et al. 1981 \\
\hline & NVSS & FIRST & PMN \\
\hline Frequency (MHz) & 1400 & 1400 & 4850 \\
\hline Sky region (J2000) & $\delta>-40^{\circ b}$ & $\begin{array}{c}7^{\mathrm{h}} 20^{\mathrm{m}}<\alpha<17^{\mathrm{h}} 20^{\mathrm{m}} ;+22.2<\delta<+57^{\circ} .5 \\
21^{\mathrm{h}} 20^{\mathrm{m}}<\alpha<3^{\mathrm{h}} 20^{\mathrm{m}} ;-2.5<\delta<+1.6\end{array}$ & $-87.5<\delta<+10^{\circ}$ \\
\hline \# of sources & 1689515 & 437429 & 50814 \\
\hline Resolution & $45^{\prime \prime} \times 45^{\prime \prime}$ & $5^{\prime \prime} \times 5^{\prime \prime}$ & $4^{\prime} .2$ \\
\hline $\begin{array}{l}\text { Position uncertainty } \\
\text { (strong sources) }\end{array}$ & $1^{\prime \prime}$ & 0.1 & $\sim 45^{\prime \prime}$ \\
\hline RMS noise & $0.5 \mathrm{mJy}$ & $0.15 \mathrm{mJy}$ & $\sim 8 \mathrm{mJy}$ \\
\hline Flux density limit & $2.5 \mathrm{mJy}$ & $1 \mathrm{mJy}$ & $20 \mathrm{mJy}$ \\
\hline Reference & Condon et al. 1998 & Becker et al. 1995 & Griffith \& Wright 1993 \\
\hline
\end{tabular}

${ }^{a}$ The Texas interferometer has a complicated beam. However, sources with separations between $10^{\prime \prime}$ and $2^{\prime}$ can be successfully modeled as doubles, and will have a single entry in the catalog. See Douglas et al. (1996) for details.

${ }^{b}$ Some small gaps are not covered. They are listed on the NVSS homepage (1998 January 19 version).

With the advent of several new deep all-sky surveys (Sect. 2), it is now possible for the first time to construct a well defined all-sky USS sample with optimized selection criteria to find large numbers of $z>3$ radio galaxies. In this paper, we describe the construction of such a sample, and present high resolution radio observations needed to determine accurate positions and morphologies. This information is essential for the optical and near-IR identifications, and subsequent optical spectroscopy of a significant sub-set of our sample, which will be described in future papers. The organization of the paper is as follows: we describe the radio surveys we used in Sect. 2 and define our samples in Sect. 3. We present and discuss our radio observations in Sect. 4, and present our conclusions in Sect. 5 .

\section{Description of the radio surveys}

During the past years, several all-sky radio-surveys have become available (Table 1 ), which are $1-2$ orders of magnitude more sensitive than previous surveys at similar frequencies (Fig. 2). The combination of these new surveys allows us to define for the first time a large sample of USS sources that covers the whole sky ${ }^{3}$ in both hemispheres. We list the main survey parameters in Table 1 . In this section, we will briefly discuss the usefulness of these new radio surveys for the construction of USS samples.

\footnotetext{
3 To facilitate optical follow-up, we will exclude the Galactic plane at $|b|<15^{\circ}$.
}

\subsection{WENSS}

The Westerbork Northern Sky Survey (WENSS; Rengelink et al. 1997) at $325 \mathrm{MHz}$ is the deepest low-frequency survey with a large sky coverage $(3.14 \mathrm{sr})$. We used the WENSS to define the largest, and most complete USS sample to date, covering the entire sky North of declination $+29^{\circ}$. We used version 1.0 of the main and polar WENSS catalogs. A small area is covered by both these catalogs; we selected only the sources from the main catalog in this overlapping area.

\subsection{Texas}

The Texas survey, made with the Texas interferometer from 1974 to 1983 (Douglas et al. 1996), covers 9.63 steradians at a frequency of $365 \mathrm{MHz}$ with a limiting flux density about ten times higher than that of the WENSS. The Texas interferometer's $3.5 \mathrm{~km}$ maximum baseline provides $<1^{\prime \prime}$ positional accuracy, but its poor $u v$-coverage leads to irregular beamshapes and lobe-shifts, hampering accurate modeling of extended sources. A detailed discussion of these complications can be found in Douglas et al. (1996). To minimize these problems, we have selected only the $40.9 \%$ sources that are well modeled (listed with a "+++" flag in the catalog). This selection excludes primarily $S_{365} \lesssim 700$ mJy sources (Fig. 3), but even at $S_{365} \gtrsim 700 \mathrm{mJy}$, one out of three sources is excluded by this criterion. Douglas et al. (1996) have calculated the 


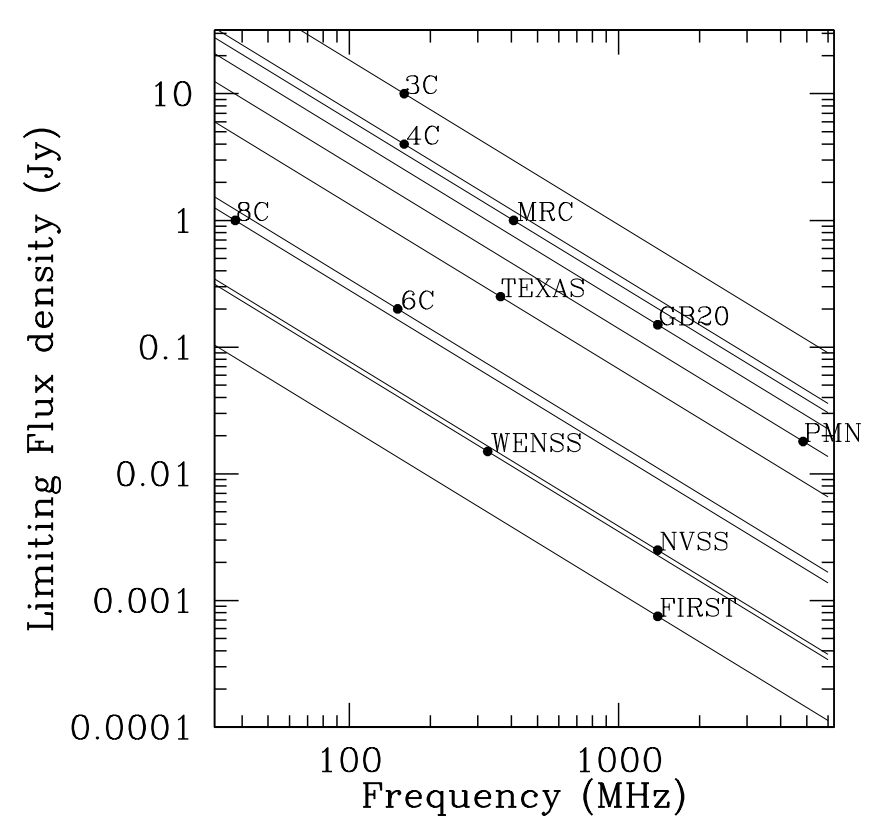

Fig. 2. Limiting flux density plotted for all major radio surveys. Lines are of constant spectral indices of -1.3 . Note that WENSS, NVSS and FIRST have flux density limits $\sim 100$ times deeper than previous surveys at comparable wavelengths

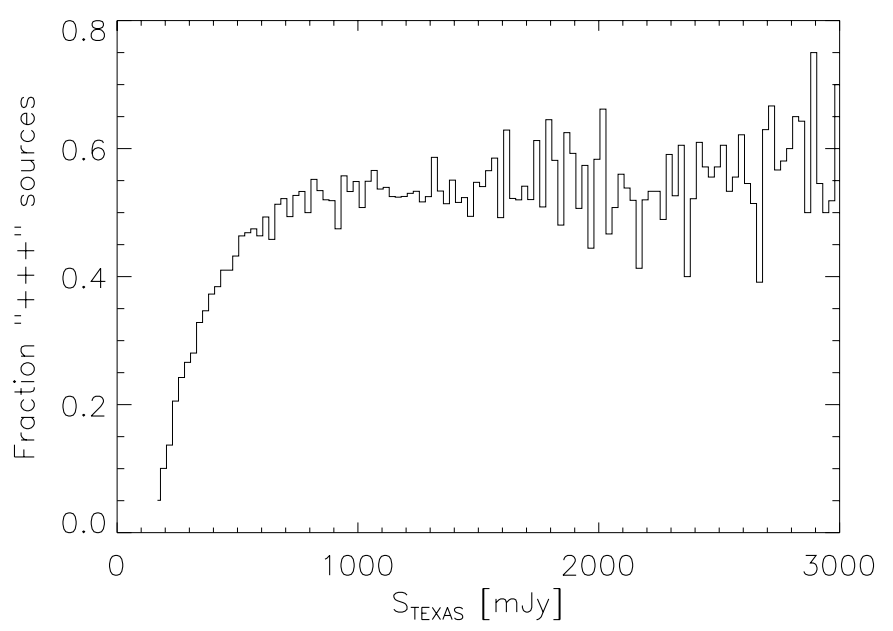

Fig. 3. Fraction of sources with " +++ " flag in the Texas catalog (see text) as a function of Texas $S_{365}$ flux density. Note that the selection of " +++ " sources excludes primarily sources with $S_{365} \lesssim 700$ mJy

completeness above flux density $S$ of the Texas catalog (defined as the fraction of sources with true flux density greater than $S$ which appear in the catalog) by comparing the Texas with the MRC (Large et al. 1981) and a variety of other low-frequency catalogs. They found that the completeness varies with declination (because the survey was done in declination strips over a large time span), and an expected increase in completeness at higher flux densities. In Table 2, we reproduce their completeness table, extended with the values after the " +++ " selection.
Table 2. Completeness of the Texas survey

\begin{tabular}{lcc}
\hline Limiting flux density & all sources & "+++" sources \\
\hline $250 \mathrm{mJy}$ & 0.8 & 0.2 \\
$350 \mathrm{mJy}$ & 0.88 & 0.28 \\
$500 \mathrm{mJy}$ & 0.92 & 0.40 \\
$750 \mathrm{mJy}$ & 0.96 & 0.51 \\
$1 \mathrm{Jy}$ & 0.96 & 0.50 \\
\hline
\end{tabular}

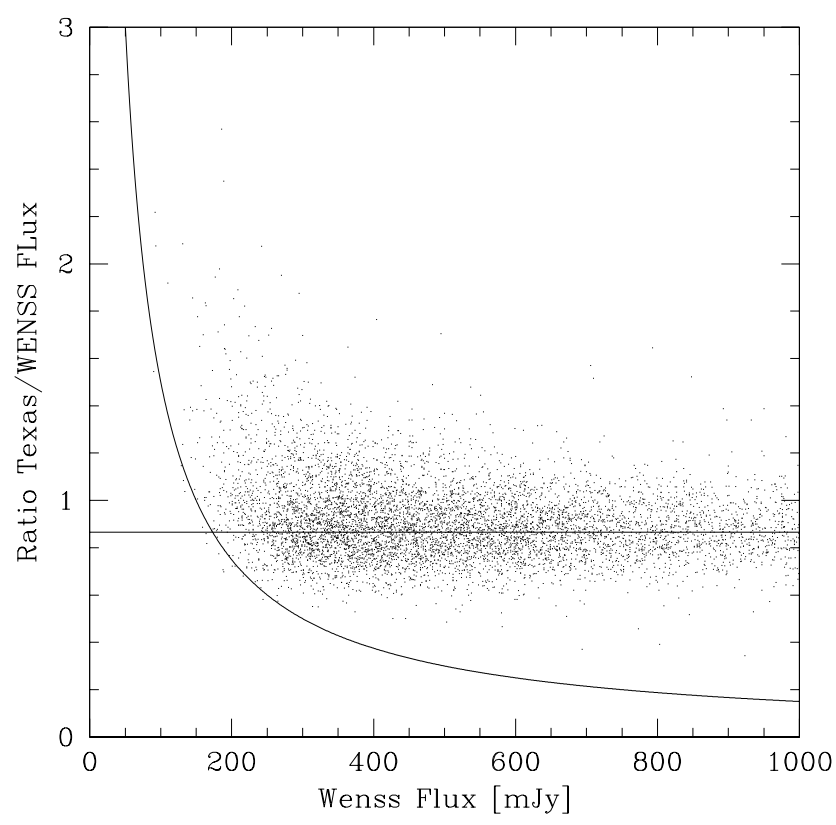

Fig. 4. Ratio of integrated Texas over WENSS flux density against integrated WENSS flux density. Only Texas "+++" sources are plotted. The horizontal line is the expected ratio 0.865 due to the $40 \mathrm{MHz}$ difference in central frequency, and assuming a spectral index of $\alpha_{365}^{1400}=-0.88$. The curved line indicates a $150 \mathrm{mJy}$ flux density limit in the Texas catalog. Note the increasing amount of overestimated Texas flux densities with decreasing flux density $S_{\text {WENSS }}<400$ mJy

To examine the reliability of the listed flux densities, and to check to what extent the " +++ " selection has removed the spurious sources from the catalog, we have correlated the Texas "+++" sources with WENSS, NVSS and FIRST. In Fig. 4, we compare the Texas flux densities with those of the WENSS. At $S_{325} \gtrsim 500 \mathrm{mJy}$, the ratio of the flux densities is closely distributed around 0.9 . This ratio is what we expect due to the $40 \mathrm{MHz}$ central frequency difference between the two surveys and assuming a spectral index $\alpha_{365}^{1400}=-0.879$ (the median of the Texas-NVSS spectral indices). At $S_{325} \lesssim 500 \mathrm{mJy}$, the number of sources in the Texas catalog which are brighter than in WENSS catalog increases with decreasing flux density. This can be explained by the "up-scattering" of sources near the flux limit of the Texas catalog (i.e. only sources intrinsically brighter than $S_{365}=150 \mathrm{mJy}$ will be detected, but no $S_{365}<150$ mJy sources with a large 
positive flux density measurement error). The result of this on a USS sample based on the Texas survey and correlated with a higher frequency survey (such as the NVSS), will be that with lower $S_{365}$, we will find more sources whose spectral indices appear steeper than they really are.

We also examined the dependence of the ratio Texas/WENSS flux density on angular size, determined from the FIRST survey (see Sect. 2.1.4). We found no significant residual variation of the flux density ratio at sizes between $5^{\prime \prime}$ and $2^{\prime}$.

In Fig. 5b, we plot the density of NVSS sources around a Texas source (see also Sect. 2.2.5). The width of the over-density peak $\left(\sim 10^{\prime \prime}\right)$ is due to the positional inaccuracies in the Texas and NVSS catalogues. However, the very broad tail of sources between $20^{\prime \prime}$ and $110^{\prime \prime}$ and the secondary peak coinciding with the fringe separation at $73^{\prime \prime}$ indicates that the " +++ " selection did not remove all spurious sources from the catalog.

In summary, after the selection of " +++ " sources, the Texas catalog still contains $<5 \%$ spurious sources (Douglas et al. 1996), probably due to residual lobe-shifted sources. Our comparison of the Texas flux densities with those of the WENSS survey shows that the differences are consistent with the errors quoted in the catalog. The selection of the Texas catalog with only " +++ " sources is thus $>95 \%$ reliable, but only $\sim 40 \%$ complete.

\section{3. $N V S S$}

The NRAO VLA Sky Survey (NVSS; Condon et al. 1998) covers the 10.3 steradians north of $-40^{\circ}$ at $1.4 \mathrm{GHz}$, and reaches a 50 times lower limiting flux density than previous large area $1.4 \mathrm{GHz}$ surveys. At the flux density levels we are using $\left(S_{1400}>10 \mathrm{mJy}\right)$, the catalog is virtually complete. Because the NVSS resolution is comparable to that of the WENSS and Texas surveys, and its sky coverage is large, we use the NVSS to determine the spectral indices in our USS samples based on the WENSS and Texas surveys. The final NVSS catalog was not yet completed at the time of our USS sample construction. For our final sample, presented in this paper, we use the 1998 January 19 version. This version still lacks data in a small number of regions of the sky (listed on the NVSS homepage). As a result, the sky coverage of the area listed in Table 3 is only $99.77 \%$.

\subsection{FIRST}

The Faint Images of Radio Sky at Twenty centimeters (FIRST, Becker et al. 1995) survey is currently being made with the VLA in the B-array at $1.4 \mathrm{GHz}$, and has a limiting flux density three times deeper than the NVSS. We used the 1998 February 4 version of the catalog, covering 1.45 steradians. As noted by Becker et al. (1995), the photometry for extended sources in FIRST might be less reliable than that of the NVSS, due to the $9 \times$ higher resolution, which could underestimate large-scale diffuse radio emission. As the FIRST area is completely covered by the NVSS, we will consistently use NVSS flux densities for our spectral index calculation. The main advantages of FIRST over NVSS for our purposes are the much better positional accuracy $(<0.5)$ and the higher $\left(5^{\prime \prime}\right)$ resolution. This combination allows the identification of even the very faint $(R>20)$ optical counterparts of radio sources. Additionally, the fainter detection limit of the FIRST allows an extra check on the flux densities of compact sources.

\section{5. $M R C$}

The Molonglo Reference Catalog of radio sources (Large et al. 1981) at $408 \mathrm{MHz}$ is presently the most sensitive low-frequency catalog with reasonable positional accuracy that covers the deep southern hemisphere, $\delta<-35^{\circ}$. We will use this catalog in combination with the PMN survey (see below) to define the first USS sample at $\delta<-40^{\circ}$.

\section{6. $P M N$}

The Parkes-MIT-NRAO (PMN) survey is a combination of 4 strips observed with the Parkes telescope at $4.85 \mathrm{GHz}$. The strips cover different parts of the sky, each with a slightly different limiting flux density. The regions are: southern $\left(-87^{\circ} .5<\delta<-37^{\circ}\right.$, Wright et al. 1994), zenith $\left(-37^{\circ}<\delta<-29^{\circ}\right.$, Wright et al. 1996), tropical $\left(-29^{\circ}<\delta<-9.5\right.$, Griffith et al. 1994), and equatorial $\left(-9.5<\delta<+10^{\circ}\right.$, Griffith et al. 1995). For our southern hemisphere sample, we have used the southern and zenith catalogs to find USS sources at $\delta<-30^{\circ}$.

\section{USS samples}

Figure 2 shows that the surveys described above have very compatible flux density limits for defining samples of USS sources. At the same time, their sky coverage is larger and more uniform than previous surveys used for USS sample construction (Wieringa \& Katgert 1992; Röttgering et al. 1994; Chambers et al. 1996a; Blundell et al. 1998; Rengelink 1998; Pursimo et al. 1999; Pedani \& Grueff 1999; Andernach et al. 2000). We selected the deepest low and high frequency survey available at each part of the sky. For a small region $-35^{\circ}<\delta<-30^{\circ}$ which is covered by both Texas and MRC, we used both surveys. This resulted in a more complete samples since the lower sensitivity of the PMN survey in the zenith strip (see Sect. 2.6) is partly compensated by the (albeit incomplete) Texas survey. To avoid problems with high Galactic extinction during optical imaging and spectroscopy, all regions at 
a)

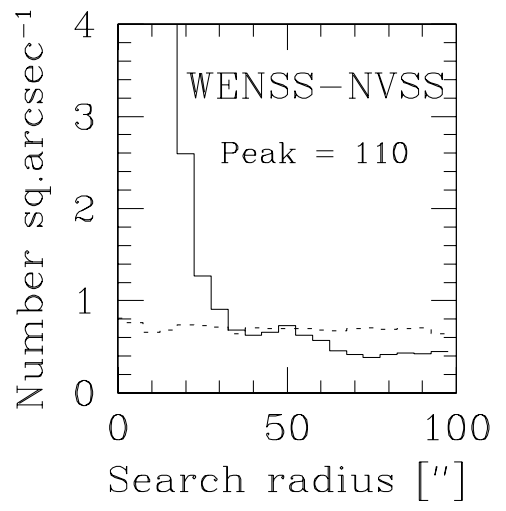

b)

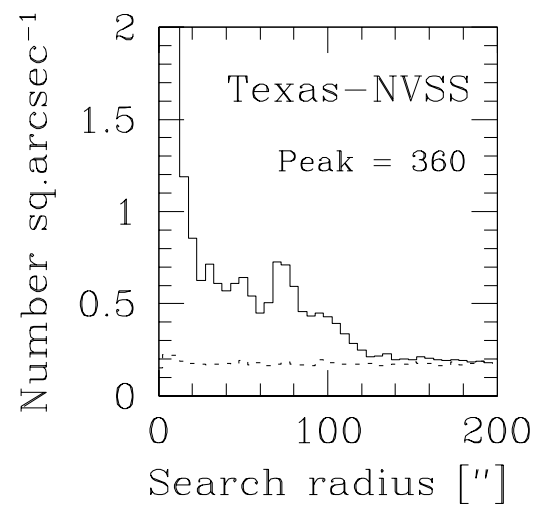

c)

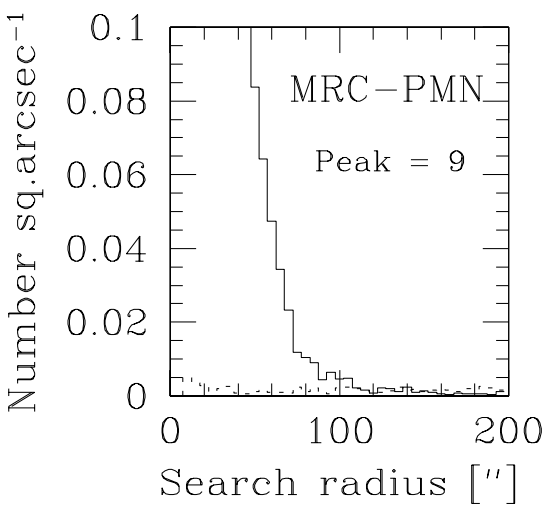

Fig. 5. The density of sources from the high frequency catalog used in the correlation (NVSS or PMN) around sources from the low frequency catalog (WENSS, Texas or MRC) as a function of search radius. The dotted line represents the distribution of confusion sources (see text). The apparent under-density of WENSS-NVSS sources at search radii $\gtrsim 60^{\prime \prime}$ is due to the grouping of multiple component sources in the $\sim 1^{\prime}$ resolution WENSS. Note the plateau and secondary peak around the $73^{\prime \prime}$ Texas fringe separation in the Texas-NVSS correlation and the much larger uncertainty in the MRC-PMN correlation

Galactic latitude $|b|<15^{\circ}$ were excluded ${ }^{4}$, as well as the area within $7^{\circ}$ of the LMC and SMC. This resulted in three USS samples that cover a total of 9.4 steradians (Fig. 6).

We designate the USS samples by a two-letter name, using the first letter of their low- and high-frequency contributing surveys. Sources from these samples are named with this 2-letter prefix followed by their IAU J2000-names using the positions from the NVSS catalog (WN and TN samples) or the MRC catalog (MP sample). We did not rename the sources after a more accurate position from our radio observations or from the FIRST survey. The sample definitions are summarized in Table 3.

\subsection{Survey combination issues}

We first discuss the problems that arise when combining radio surveys with different resolutions and positional uncertainties.

\subsubsection{Correlation search radius}

Due to the positional uncertainties and resolution differences between radio surveys, in general the same source will be listed with slightly different positions in the catalogs.

To empirically determine the search radius within which to accept sources in 2 catalogs to be the same, we compared the density of objects around the position listed in the low-frequency survey (which has lower resolution) with the expected number of random correlations in each sample (三 confusion sources). To determine this number

\footnotetext{
4 This also reduces the number of Galactic pulsars in our sample (see Sect. 4.7.2).
}

as a function of distance from the position in the most accurate catalog, we created a random position catalog by shifting one of the input catalogs by $1^{\circ}$ in declination, and made a correlation with this shifted catalog. The density of sources as a function of distance from the un-shifted catalog then represents the expected number of confusion sources as a function of radial distance. In Fig. 5, we plot for each of our three samples the observed density around these sources with this confusion distribution over-plotted. The correlation search radius should thus be chosen at a distance small enough for the density of confusion sources to be negligible.

We decided to adopt the radius where the density of real sources is at least ten times higher that the density of confusion sources as the search radius for our sample construction, except for the WN sample (would be $15^{\prime \prime}$ ) where we chose the same radius as for the TN sample $\left(10^{\prime \prime}\right)$. The later was done for consistency between both samples. Because of the five times lower resolution and source densities in the MRC and PMN surveys, the search radius of the MP sample is eight times larger. Summarized, the search radii we used are $10^{\prime \prime}$ for $\mathrm{WN}$ and $\mathrm{TN}$, and $80^{\prime \prime}$ for MP.

\subsubsection{Angular size}

In order to minimize errors in the spectral indices due to different resolutions and missing flux on large angular scales in the composing surveys, we have only considered sources which are not resolved into different components in the composing surveys. Effectively, this imposes an angular size cutoff of $\sim 1^{\prime}$ to the $\mathrm{WN}, \sim 2^{\prime}$ to the TN sample and $\sim 4^{\prime}$ to the MP sample. We deliberately did not choose a smaller angular size cutoff (as e.g. Blundell et al. 1998, 
Table 3. USS samples

\begin{tabular}{|c|c|c|c|c|c|c|c|c|}
\hline Sample & Sky Area & $\begin{array}{c}\text { Density } \\
\mathrm{sr}^{-1}\end{array}$ & Spectral Index & Search Radius & $\begin{array}{c}\text { Flux Limit } \\
\text { mJy }\end{array}$ & $\mathrm{C}^{a}$ & $\mathrm{R}^{a}$ & \# of Sources \\
\hline WN & $29^{\circ}<\delta<75^{\circ},|b|>15^{\circ b}$ & 151 & $\alpha_{325}^{1400} \leq-1.30$ & $10^{\prime \prime}$ & $S_{1400}>10$ & $96 \%$ & $90 \%$ & 343 \\
\hline $\mathrm{TN}$ & $-35^{\circ}<\delta<29^{\circ},|b|>15^{\circ b}$ & $48^{c}$ & $\alpha_{365}^{1400} \leq-1.30$ & $10^{\prime \prime}$ & $S_{1400}>10$ & $97 \%^{c}$ & $93 \%$ & 268 \\
\hline MP & $\delta<-30^{\circ},|b|>15^{\circ}$ & 26 & $\alpha_{408}^{4800} \leq-1.20$ & $80^{\prime \prime}$ & $S_{408}>700 ; S_{4850}>35$ & $100 \%$ & $100 \%$ & 58 \\
\hline
\end{tabular}

${ }^{a} \mathrm{C}=$ completeness and $\mathrm{R}=$ reliability accounting only for scattering across the spectral index limit (see Sect. 3.3.1).

${ }^{b}$ Coverage is only $99.7 \%$ because some small patches of sky we not covered at the time of writing. They are listed on the NVSS homepage (1998 January 19 version).

${ }^{c}$ Because we selected only problem free sources from the Texas survey, the effective completness of the TN sample is $\sim 30 \%$.

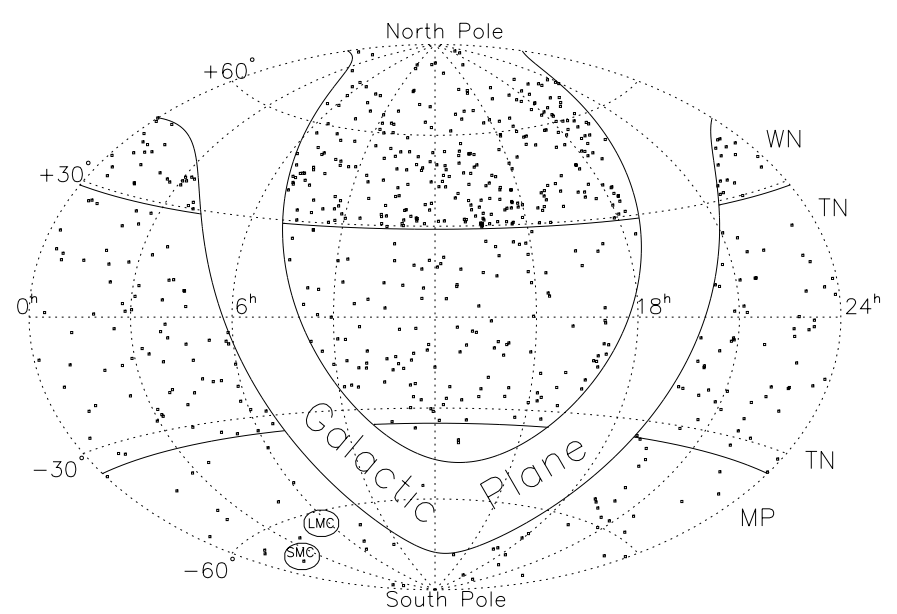

Fig. 6. Sky coverage of our 3 USS samples. Constant declination lines denote the boundaries between our the WN and TN and between the TN and MP samples, as indicated on the right. Note the difference in source density and the exclusion of the Galactic Plane

did for the $6 \mathrm{C}^{*}$ sample), because (1) higher resolution angular size information is only available in the area covered by the FIRST survey, and (2) even a $15^{\prime \prime}$ cutoff would only reduce the number of sources by $30 \%$, while it would definitely exclude several HzRGs from the sample. For example, in the 4C USS sample (Chambers et al. 1996b), three out of eight $z>2$ radio galaxies have angular sizes $>15^{\prime \prime}$.

We think that our $\sim 1^{\prime}$ angular size cutoff will exclude almost no HzRGs, because the largest angular size for $z>2$ radio galaxies in the literature is $53^{\prime \prime}$ (4C 23.56 at $z=2.479$; Chambers et al. 1996a; Carilli et al. 1997), while all $45 z>2.5$ radio galaxies with good radio maps are $<35^{\prime \prime}$ (Carilli et al. 1997). Although the sample of known $z>2$ radio galaxies is affected by angular size selection effects, very few HzRGs larger than $1^{\prime}$ would be expected.

The main incompleteness of our USS sample stems from the spectral index cutoff and the flux limit (Sect. 3.2). However, our flux limit $\left(S_{1400}=10 \mathrm{mJy}\right)$ is low enough to break most of the redshift-radio power degeneracy at $z>2$. To achieve this with flux limited samples, multiple samples are needed (e.g. Blundell et al. 1999).

\subsection{Sample definition}

\subsubsection{WENSS-NVSS (WN) sample}

A correlation of the WENSS and NVSS catalogs with a search radius of $10^{\prime \prime}$ centered on the WENSS position (see Sect. 3.1.1) provides spectral indices for $\sim 143000$ sources. Even with a very steep $\alpha_{325}^{1400} \leq-1.30$ spectral index criterion, we would still have 768 sources in our sample. To facilitate follow-up radio observations, and to increase the accuracy of the derived spectral indices (see Sect. 3.3.1), we have selected only NVSS sources with $S_{1400}>10 \mathrm{mJy}$. Because the space density of the highest redshift galaxies is low, it is important not to limit the sample area (see e.g. Rawlings et al. 1998) to further reduce the number of sources in our sample. Because the NVSS has a slightly higher resolution than the WENSS ( $45^{\prime \prime}$ compared to $54^{\prime \prime} \times 54^{\prime \prime} \operatorname{cosec} \delta$ ), some WENSS sources have more than one associated NVSS source. We have rejected the 11 WN sources that have a second NVSS source within one WENSS beam. Instead of the nominal WENSS beam $\left(54^{\prime \prime} \times 54^{\prime \prime} \operatorname{cosec} \delta\right)$, we have used a circular $72^{\prime \prime}$ WENSS beam, corresponding to the major axis of the beam at $\delta=48^{\circ}$, the position that divides the WN sample into equal numbers to the North and South. The final WN sample contains 343 sources.

\subsubsection{Texas-NVSS (TN) sample}

Because the Texas and NVSS both have a large skycoverage, the area covered by the TN sample includes $90 \%$ of the WN area. In the region $\delta>29^{\circ}$, we have based our sample on the WENSS, since it does not suffer from lobe-shift problems and reaches ten times lower flux densities than the Texas survey (Sect. 2.2). In the remaining 5.28 steradians South of declination $+29^{\circ}$, we have spectral indices for $\sim 25200$ sources. Again, we used a $10^{\prime \prime}$ search radius (see Sect. 3.1.1), and for the same reason as in the WN sample we selected only NVSS sources with $S_{1400}>10 \mathrm{mJy}$. Combined with the $\alpha_{365}^{1400} \leq-1.30$ criterion, the number of USS TN sources is 285. As for the WN sample, we further excluded sources with more than one $S_{1400}>10$ mJy NVSS source within $60^{\prime \prime}$ around the TEXAS position, leaving 268 sources in the final TN sample. We remind (see Sect. 2.2) that the selection of the 
TEXAS survey we used is only $\sim 40 \%$ complete with a strong dependence on flux density. Using the values from Table 2, we estimate that the completeness of our $\mathrm{TN}$ sample is $\sim 30 \%$.

\subsubsection{MRC-PMN (MP) sample}

In the overlapping area, we preferred the TN over the MP sample for the superior positional accuracies and resolutions of both Texas and NVSS compared to MRC or PMN. Because the MRC survey has a low source density, we would have only $13 \mathrm{MP}$ sources with $\alpha_{408}^{4850} \leq-1.30$. We therefore relaxed this selection criterion to $\alpha_{408}^{4850} \leq-1.20$, yielding a total sample of 58 sources in the deep South $\left(\delta<-30^{\circ}\right)$.

\subsection{Discussion}

\subsubsection{Spectral index errors}

We have listed the errors in the spectral indices due to flux density errors in the catalogs in Tables A.1 to A.3. The WN and TN samples have the most accurate spectral indices: the median spectral index errors are $\Delta \bar{\alpha}_{325}^{1400}=0.04$ for WN sources and $\Delta \bar{\alpha}_{365}^{1400}=0.04\left(S_{365}>1 \mathrm{Jy}\right)$ to $0.07\left(S_{365}>150 \mathrm{mJy}\right)$ for TN sources. For the MP sample, $\Delta \bar{\alpha}_{408}^{4850} \approx 0.1$, with little dependence on flux density $\left(S_{408}>750 \mathrm{mJy}\right)$.

Because our sample selects the sources in the steep tail of the spectral index distribution (Figs. 7 and 8), there will be more sources with an intrinsic spectral index flatter than our cutoff spectral index that get scattered into our sample by measurement errors than there will be sources with intrinsic spectral index steeper than the cutoff that get scattered out of our sample.

Following the method of Rengelink (1998), we fitted the steep tail between $-1.60<\alpha<-1.0$ with a Gaussian function. For each of our three samples, we generated a mock sample drawn from this distribution, and added measurement errors by convolving this true spectral index distribution with a Gaussian distribution with as standard deviation the mean error of the spectral indices. The WN mock sample predicts that $13 \alpha_{325}^{1400}<-1.30$ sources get scattered out of the sample while $36 \alpha_{325}^{1400}>-1.30$ sources get scattered into the USS sample. Thus, the WN sample is $96 \%$ complete and $90 \%$ reliable. For the TN sample, we expect to loose $7 \alpha_{365}^{1400}<-1.30$ sources $^{5}$, and have 18 contaminating $\alpha_{365}^{1400}>-1.30$ sources. The completeness is thus $97 \%$ and the reliability $93 \%$. For the MP sample, this spectral index scattering is negligible, because there are too few sources in the steep spectral index tail.

\footnotetext{
${ }^{5}$ Only due to the spectral index cutoff, the sample has more important incompleteness factors; see Sect. 2.2.
}

Our reliability and completeness are significantly better than the values of $\sim 75 \%$ and $\sim 50 \%$ of Rengelink (1998) because (1) our spectral indices are more accurate because they were determined from a wider frequency interval than the $325-610 \mathrm{MHz}$ used by Rengelink (1998), and (2) our sample has a steeper cutoff spectral index, where the spectral index distribution function contains fewer sources and has a shallower slope, leading to fewer sources that can scatter in or out of the sample.

\subsubsection{Spectral index distributions}

Using the 143000 spectral indices from the WENSS-NVSS correlation, we examined the flux density dependence of the steep and flat spectrum sources. Selecting sources with $S_{325}>50 \mathrm{mJy}$ or $S_{1400}>100 \mathrm{mJy}$ assures that we will detect all sources with $\alpha_{325}^{1400}>\frac{\ln \left(S_{\mathrm{NVSS}}^{\lim } / 50\right)}{\ln (325 / 1400)}=-1.82$ or $\alpha_{325}^{1400}<\frac{\ln \left(S_{\mathrm{WENSS}}^{\lim } / 100\right)}{\ln (325 / 1400)}=0.82$ respectively, where $S_{\mathrm{NVSS}}^{\lim }=3.5 \mathrm{mJy}$ and $S_{\mathrm{WENSS}}^{\lim }=30 \mathrm{mJy}$ are the lowest flux densities where the NVSS and WENSS are complete (Condon et al. 1998; Rengelink et al. 1997). The results shown in Fig. 7 therefore reflect only the effect of a different selection frequency. Two populations are present in both the $S_{325}$ and $S_{1400}$ selected distributions. The peaks of the steep and flat populations at $\bar{\alpha}_{325}^{1400} \approx-0.8$ and $\bar{\alpha}_{325}^{1400} \approx-0.4$ do not show significant shifts over three orders of magnitude in flux density. This is consistent with the results that have been found at $4.8 \mathrm{GHz}$ (Witzel et al. 1979; Machalski \& Ryś 1981; Owen et al. 1983), with the exception that their $\bar{\alpha}_{1400}^{4800} \approx 0.0$ for the flat spectrum component is flatter than the $\bar{\alpha}_{325}^{1400} \approx-0.4$ we found. However, we find that the relative contribution of the flat spectrum component increases from $25 \%$ at $S_{1400}>0.1 \mathrm{Jy}$ to $50 \%$ at $S_{1400}>2.5 \mathrm{Jy}$.

Because the steep- and flat-spectrum populations are best separated in the $S_{1400}>2.5 \mathrm{Jy}$ bin, we have searched the literature for identifications of all $58 S_{1400}>2.5 \mathrm{Jy}$ sources to determine the nature of both populations. All but one (3C 399, Martel et al. 1998) of the objects outside of the Galactic plane $\left(|b|>15^{\circ}\right)$ were optically identified. Of the 30 steep spectrum $\left(\alpha_{325}^{1400}<-0.6\right)$ sources, two thirds were galaxies, while the rest were quasars. Half of the flat spectrum $\left(\alpha_{325}^{1400}>-0.6\right)$ sources were quasars, $20 \%$ blazars, and $30 \%$ galaxies. Figure 7 therefore confirms that the steep and flat spectral index populations are dominated by radio galaxies and quasars respectively. We also find that while the relative strength between the steep and flat spectrum populations changes due to the selection frequency, the median spectral index and width of the population does not change significantly over three orders in magnitude of flux density. Even fainter studies would eventually start to get contamination from the faint blue galaxy population (see e.g. Windhorst et al. 1985). 

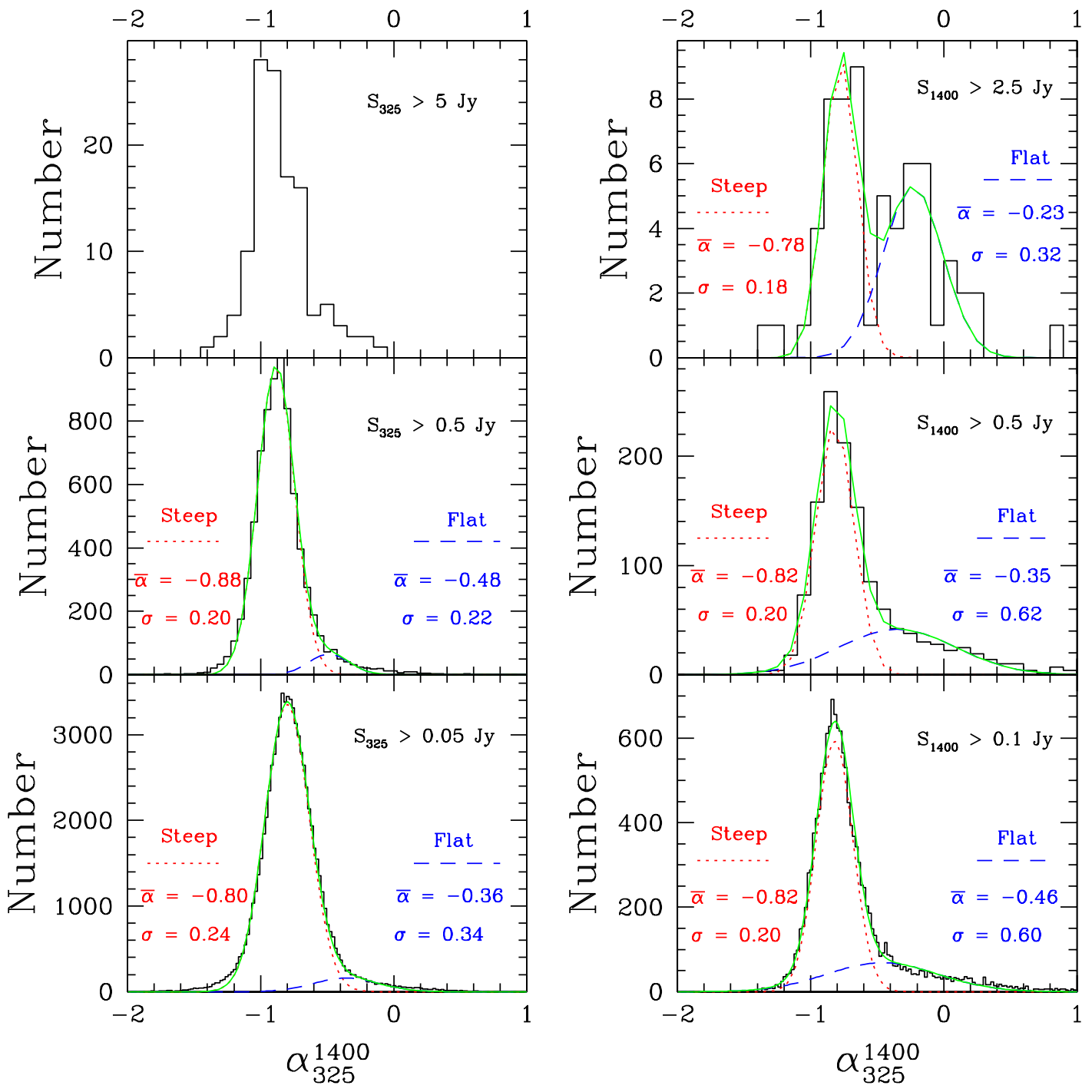

Fig. 7. Spectral index distributions from the WENSS-NVSS correlation. The left and right panels show the variation with $325 \mathrm{MHz}$ and $1.4 \mathrm{GHz}$ flux density. A low frequency selected sample is more appropriate to study the steep-spectrum population. The parameters of a two-component Gaussian fit (dotted line $=$ steep, dashed line $=$ flat) are shown in each panel. The solid line is the sum of both Gaussians

Table 4. Radio observations

\begin{tabular}{lrrrrr}
\hline UT Date & Telescope & Config. & Frequency & Resolution & \# of sources \\
\hline 1996 October 28 & VLA & A & $4.86 \mathrm{GHz}$ & $0^{\prime \prime} 3$ & $90 \mathrm{WN}, 25 \mathrm{TN}$ \\
1997 January 25 & VLA & BnA & $4.86 \mathrm{GHz}$ & $0^{\prime \prime} 6$ & $29 \mathrm{TN}$ \\
1997 March 10 & VLA & BnA & $4.885 \mathrm{GHz}$ & $0^{\prime \prime} 6$ & $8 \mathrm{TN}$ \\
1997 December 15 & ATCA & $6 \mathrm{C}$ & $1.420 \mathrm{GHz}$ & $6^{\prime \prime} \times 6^{\prime \prime} \operatorname{cosec} \delta$ & $41 \mathrm{MP}, 32 \mathrm{TN}$ \\
1998 August 12+17 & VLA & B & $4.86 \mathrm{GHz}$ & $1^{\prime \prime} .0$ & $151 \mathrm{WN}$ \\
\hline
\end{tabular}




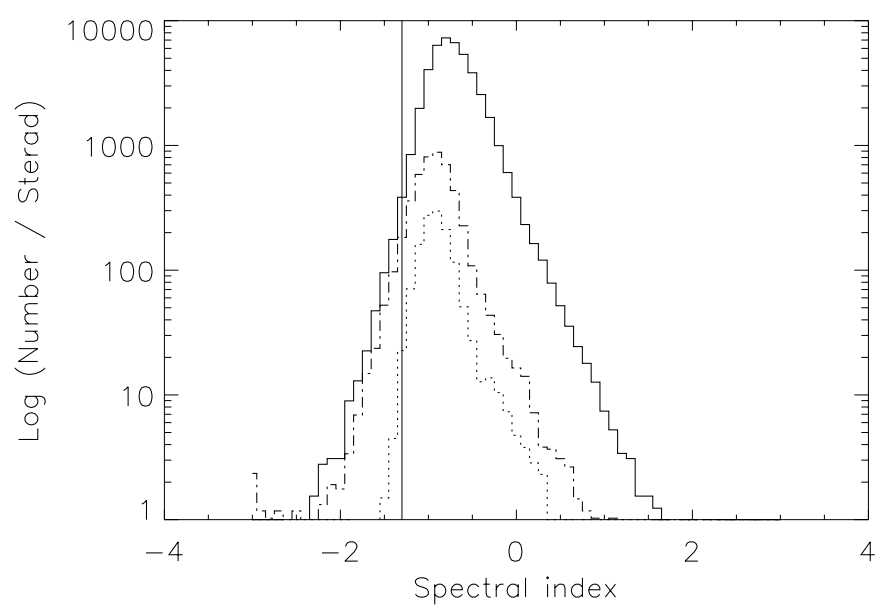

Fig. 8. Logarithmic spectral index distribution for WENSSNVSS (full line), Texas-NVSS (dot-dash line) and MRC-PMN (dotted line). The vertical line indicates the -1.3 cutoff used in our spectral index selection. Note the difference in number density and the sharper fall-off on the flat-end part of the TN and MP compared to the WN

\subsubsection{Consistency of the three USS samples}

We compare the spectral index distributions of our three USS samples in logarithmic histograms (Fig. 8). The distributions are different in two ways. First, the WENSSNVSS correlation contains nine times more sources than the Texas-NVSS, and 14 times more than the MRC-PMN correlation. Second, the shapes of the distributions are different: while the steep side of the TN sample coincides with that of the WN, its flat end part falls off much faster. The effect is so strong that it even shifts the TN peak steep-wards by $\sim 0.15$. For the MP sample, the same effect is less pronounced, though still present.

Both effects are due to the different flux density limits of the catalogs. The deeper WENSS catalog obviously contains more sources than the TEXAS or MRC catalogs, shifting the distributions vertically in Fig. 2. The relative "shortage" of flat spectrum sources in the Texas-NVSS and MRC-PMN correlations can be explained as follows. A source at the flux density limit in both WENSS and NVSS would have a spectral index of $\alpha_{325}^{1400}=-1.3$, while for Texas and NVSS this would be $\alpha_{365}^{1400}=-1.7$ (see Fig. 2). Faint NVSS sources with spectral indices flatter than these limits will thus more often get missed in the TEXAS catalog than in the WENSS catalog. This effect is even strengthened by the lower completeness at low flux densities of the Texas catalog. However, very few USS sources will be missed in either the WENSS-NVSS or Texas-NVSS correlations ${ }^{6}$. The parallel slope also indicates that the USS sources from both the WENSS-NVSS and Texas-NVSS correlations were drawn from the same

\footnotetext{
6 The TN sample will have more spurious sources at low flux density levels; see Sect. 2.2.
}

population of radio sources. We therefore expect a similar efficiency in finding HzRGs from both samples.

The MP sample has been defined using a spectral index with a much wider frequency difference. However, the observed ATCA $1.420 \mathrm{GHz}$ flux densities can be used to construct $\alpha_{408}^{1420}$. An "a posteriori" selection using $\alpha_{408}^{1420} \leq-1.30$ from out ATCA observations (see Sect. 4.2) would keep $\sim 60 \%$ of the MP sources in a WN/TN USS sample.

\section{Radio observations}

Of all the major radio surveys described in Sect. 2, only FIRST has sufficient positional accuracy and resolution for the optical identification of $R>20$ objects. We present FIRST maps of $139 \mathrm{WN}$ and $8 \mathrm{TN}$ sources in Appendices B.2 and B.4.

Outside the area covered by FIRST, we have observed all the remaining WN sources, $30 \%$ of the TN sample, and $71 \%$ of the MP sources at 0.3 to $5^{\prime \prime}$ resolution using the Very Large Array (VLA; Napier et al. 1983) and Australia Telescope Compact Array (ATCA; Frater et al. 1992) telescopes. A log of the radio observations is given in Table 4 . We observed targets for our VLA runs on the basis of declination $\left(\mathrm{A}\right.$-array for $\delta>0^{\circ}$ and $\mathrm{BnA}$-array for $\delta<0^{\circ}$ ) and sky coverage of the WN and TN samples, which were still incomplete at the time of the 1996 observations. We observed all WN, and most TN sources with the VLA, and all MP sources with the ATCA. We observed TN sources between $-31^{\circ}<\delta<-10^{\circ}$ with either VLA or ATCA, depending on the progress of the NVSS at the time of the observations.

\subsection{VLA observations and data reduction}

We observed all sources in the standard $4.86 \mathrm{GHz} C$-band with a $50 \mathrm{MHz}$ bandwidth, resulting in a resolution of $\sim 0^{\prime \prime} 3$ in the $\mathrm{A}$-array and $\sim 1^{\prime \prime}$ in the $\mathrm{BnA}$-array. We spent 5 minutes on each source, implying a theoretical rms level of $75 \mu \mathrm{Jy}$, or a ratio of total integrated signal over map noise of 110 for the weakest sources, assuming no spectral curvature beyond $1.4 \mathrm{GHz}$. We performed calibration and data editing in $\mathcal{A} \mathcal{I P S}$, the Astronomical Image Processing System from NRAO. We used 3C 286 as the primary flux calibrator in all runs. Comparison of the flux density of $3 \mathrm{C} 48$ with the predicted values indicated the absolute flux density scale was accurate up to $2 \%$. We observed nearby (within $15^{\circ}$ ) secondary flux calibrators every 15 to 20 minutes to calibrate the phases. After flagging of bad data, we spilt the $u v$-data up into separate data sets for imaging and self-calibration in DIFMAP, the Caltech difference mapping program (Sheppard et al. 1997). We used field sizes of $164^{\prime \prime}$ (A-array) or $256^{\prime \prime}$ (BnA-array) with pixel scales of 0 .' $08 /$ pixel $(\mathrm{A}-$ array) or $0 . \prime 25 /$ pixel 
(BnA-array). Even the smallest field of view is still four times larger than the resolution of the NVSS, so all components of an unresolved NVSS source will be covered.

We cleaned each source brighter than the $5 \sigma$ level, followed by a phase-only self-calibration. We repeated the latter for all sources in the field of a source. Next, we made a new model from the (self-calibrated) $u v$-data, and subsequently cleaned to the level reached before. The last stage in the mapping routine was a deep clean with a $1 \%$ gain factor over the entire field. Most of the resulting maps have noise levels in the range 75 to $100 \mu \mathrm{Jy}$, as expected.

\subsection{ATCA observations and data reduction}

We used the ATCA in the $6 \mathrm{C}$ configuration, which has a largest baseline of $6 \mathrm{~km}$. We observed at a central frequency of $1.384 \mathrm{GHz}$, which was selected to avoid local interference. We used 21 of the 30 frequency channels that had high enough signal, which resulted in an effective central frequency of $1.420 \mathrm{GHz}$, with a $84 \mathrm{MHz}$ bandwidth. In order to obtain a good $u v$-coverage, we observed each source eight to ten times for three minutes, spread in hour angle. The primary flux calibrator was the source 1934648 ; we used secondary flux calibrators within $20^{\circ}$ of the sources to calibrate the phases. We performed editing and calibration in $\mathcal{A} \mathcal{I P S}$, following standard procedures. We made maps using the automated mapping/self-calibration procedure MAPIT in $\mathcal{A I P \mathcal { S }}$. The resulting $1.420 \mathrm{GHz}$ maps (Fig. B.6.) have noise levels of $\sim 5 \mathrm{mJy}$.

\subsection{Results}

Of all 343 WN sources, 139 have FIRST maps (Appendix B.2). All remaining 204 sources were observed, and 141 were detected. The remaining $30 \%$ were too faint at $4.86 \mathrm{GHz}$ to be detected in 5 min snapshots, because their high frequency spectral index steepens more than expected, or they were over-resolved. Because they are significantly brighter, all the observed $89 \mathrm{TN}$ and $41 \mathrm{MP}$ sources were detected. We present contour maps of all the detections in Appendices B.1, B.3, B.5, and B.6 and list the source parameters in Tables A.1 to A.3.

We have subdivided our sources into 5 morphological classes, using a classification similar to that used by Röttgering et al. (1994). Note that this classification is inevitably a strong function of the resolution, which varies by a factor of 20 between the VLA A-array and the ATCA observations.

We have determined the source parameters by fitting two-dimensional Gaussian profile to all the components of a source. The results are listed in Tables A.1 to A.3 which contain:

Column 1: Name of the source in IAU J2000 format. The 2-letter prefix indicates the sample: WN: WENSSNVSS, TN: Texas-NVSS, MP: MRC-PMN.
Column 2: The integrated flux density from the low-frequency catalog.

Column 3: The integrated flux density at the intermediate frequency, determined from the NVSS for WN and $\mathrm{TN}$, or from the $1.420 \mathrm{GHz}$ ATCA observations for the MP sample.

Column 4: The integrated flux density at $4.86 \mathrm{GHz}$, determined from the VLA observations for WN and TN, and from the PMN survey for the MP sample.

Column 5: The lower frequency two-point spectral index. This is the spectral index used to define the WN and TN samples.

Column 6: The higher frequency two-point spectral index. This is the spectral index used to define the MP sample.

Column \%: Morphological classification code: single (S), double (D), triple (T) and multiple (M) component sources, and irregularly shaped diffuse (DF) sources.

Column 8: Largest angular size. For single component sources, this is the de-convolved major axis of the elliptical Gaussian, or, for unresolved sources (preceded with $<$ ), an upper limit is given by the resolution. For double, triple and multiple component sources, this is the largest separation between their components. For diffuse sources this is the maximum distance between the source boundaries defined by three times the map rms noise.

Column 9: De-convolved position angle of the radio structure, measured North through East.

Columns 10-11: J2000 coordinates, determined from the map with position code listed in Col. 12. The positions in the VLA and ATCA maps have been fitted with a single two-dimensional elliptical Gaussian. For double (D) sources, the geometric midpoint is given; for triples (T) and multiples (M), the core position is listed. For diffuse (DF) sources we list the center as determined by eye.

Column 12: Position code, indicating the origin of the morphological and positional data in Cols. 7 to 11: $\mathrm{A}=\mathrm{ATCA}, \mathrm{F}=\mathrm{FIRST}, \mathrm{M}=\mathrm{MRC}, \mathrm{N}=\mathrm{NVSS}$, and $\mathrm{V}=\mathrm{VLA}$.

\subsection{Notes on individual sources}

WN J0043+4719: The source $18^{\prime \prime}$ north of the NVSS position is not detected in the NVSS. This is therefore not a real USS source because the NVSS flux density was underestimated.

WN J0048+4137: Our VLA map probably doesn't go deep enough to detect all the flux of this source.

WN J0727+3020: The higher resolution FIRST map shows that both components of this object are indeed identified on the POSS, even though the NVSS position is too far off to satisfy our identification criterion. 
WN J0717+4611: Optical and near-IR spectroscopy revealed this object as a red quasar at $z=1.462$ (De Breuck et al. 1998b).

WN J0725+4123: The extended POSS identification suggest this source is located in a galaxy cluster.

WN J0829+3834: The NVSS position of this unresolved source is $7^{\prime \prime}(3 \sigma)$ from the FIRST position, which itself is only at $2^{\prime \prime}$ from the WENSS position.

WN J0850+4830: The difference with the NVSS position indicates that our VLA observations are not deep enough to detect a probable north-eastern component.

WN J0901+6547: This $38^{\prime \prime}$ large source is over-resolved in our VLA observations, and probably even misses flux in the NVSS, and is therefore not a real USS source.

WN J1012+3334: The bend morphology and bright optical sources to the east indicate this object is probably located in a galaxy cluster.

WN J1101+3520: The faint FIRST component 20" north of the brighter Southern component is not listed in the FIRST catalog, but is within $1^{\prime \prime}$ of a faint optical object. This might be the core of a $70^{\prime \prime}$ triple source.

WN J1152+3732: The distorted radio morphology and bright, extended POSS identification suggest this source is located in a galaxy cluster.

WN J1232+4621: This optically identified and diffuse radio source suggest this source is located in a galaxy cluster. WN J1314+3515: The diffuse radio source appears marginally detected on the POSS.

WN J1329+3046A,B, WN J1330+3037, WN J1332+3009

\& WN J1333+3037: The noise in the FIRST image is almost ten times higher than average due to the proximity of the $S_{1400}=15$ Jy source $3 \mathrm{C} 286$.

WN J1330+5344: The difference with the NVSS position indicates that our VLA observations are not deep enough to detect a probable south-eastern component.

WN J1335+3222: Although the source appears much like the hotspot of a larger source with the core $90^{\prime \prime}$ to the east, no other hotspot is detected in the FIRST within $5^{\prime}$. WN J1359+7446: The extended POSS identification suggests this source is located in a galaxy cluster.

WN J1440+3707: The equally bright galaxy $30^{\prime \prime}$ south of the POSS identification suggests that this source is located in a galaxy cluster.

WN J1509+5905: The difference with the NVSS position indicates that our VLA observations are not deep enough to detect a probable western component.

WN J1628+3932: This is the well studied galaxy NGC 6166 in the galaxy cluster Abell 2199 (e.g. Zabludoff et al. 1993).

WN J1509+5905: The difference with the NVSS position indicates that our VLA observations are not deep enough to detect a probable west-south-western component.

WN J1821+3601: The source $35^{\prime \prime}$ south-west of the NVSS position is not detected in the NVSS. This is therefore not a real USS source because the NVSS flux density was underestimated.
WN J1832+5354: The source $19^{\prime \prime}$ north-east of the NVSS position is not detected in the NVSS. This is therefore not a real USS source because the NVSS flux density was underestimated.

WN J1852+5711: The extended POSS identification suggests this source is located in a galaxy cluster.

WN J2313+3842: The extended POSS identification suggests this source is located in a galaxy cluster.

TN J0233+2349: This is probably the north-western hotspot of a $35^{\prime \prime}$ source, with the south-eastern component barely detected in our VLA map.

TN J0309-2425: We have classified this source as a $13^{\prime \prime}$ double, but the western component might also be the core of a $45^{\prime \prime}$ source, with the other hotspot around $\alpha=3^{\mathrm{h}} 9^{\mathrm{m}} 10^{\mathrm{s}}, \delta=-24^{\circ} 25^{\prime} 50^{\prime \prime}$.

TN J0349-1207: The core-dominated structure is reminiscent of the red quasar WN J0717+4611.

TN J0352-0355: This is probably the south-western hotspot of a $30^{\prime \prime}$ source.

TN J0837-1053: Given the 10" difference between the positions of the NVSS and diffuse VLA source, this is probably the northern component of a larger source.

TN J0408-2418: This is the $z=2.44$ source MRC 0406-244 (McCarthy et al. 1996). The bright object on the POSS is a foreground star to the north-east of the $R=22.7$ galaxy.

TN J0443-1212: Using the higher resolution VLA image, we can identify this radio source with a faint object on the POSS.

TN J2106-2405: This is the $z=2.491$ source MRC 2104242 (McCarthy et al. 1996). The identification is an $R=$ 22.7 object, not the star to the north-north-west of the NVSS position.

\subsection{Radio spectra and spectral curvature}

We have used the CATS database at the Special Astronomical Observatory (Verkhodanov et al. 1997) to search for all published radio measurements of the sources in our samples. In Appendix C, we show the radio spectra for all sources with flux density information for more than two frequencies (the $S_{4860}$ points from our VLA observations are also included). These figures show that most radio spectra have curved spectra, with flatter spectral indices below our selection frequencies, as has been seen in previous USS studies (see e.g. Röttgering et al. 1994; Blundell et al. 1998).

This low frequency flattening and high frequency steepening is obvious in the radio "color-color diagrams" of the WN sample (Fig. 9). The median spectral index at low frequencies $(\nu<325 \mathrm{MHz})$ is -1.16 , while the median $\bar{\alpha}_{325}^{1400}=-1.38$. At higher frequencies $(\nu>1400 \mathrm{MHz})$, the steepening continues to a median $\bar{\alpha}_{1400}^{4850}=-1.44$. Note that the real value of the latter is probably even 

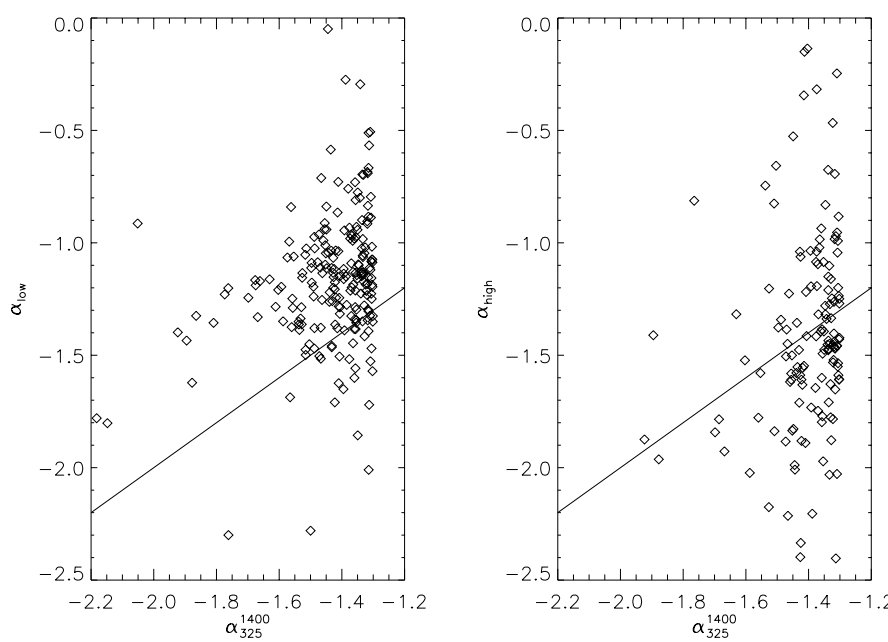

Fig. 9. Radio "color-color" plots for the WN sample. The abscissa is the $\alpha_{325}^{1400}$ spectral index used to construct the sample. The ordinates are the low-frequency spectral indices determined from the $8 \mathrm{C}(38 \mathrm{MHz}$, Rees 1990$)$ or $6 \mathrm{C}(151 \mathrm{MHz}$, Hales et al. 1993) and the $325 \mathrm{MHz}$ WENSS (left panel), and the high frequency spectral index determined between the 1.4 GHz NVSS and our 4.86 GHz VLA observations (right panel). The line in each panel indicates a straight power law spectrum. Note the unequal number of points on either side of these lines, indicating substantial spectral curvature

Table 5. Radio structure distribution

\begin{tabular}{|c|c|c|c|c|}
\hline \multirow[b]{2}{*}{ Morphology } & \multicolumn{4}{|c|}{ USS Samples } \\
\hline & $\overline{\mathrm{WN}}$ & $\mathrm{TN}$ & MP & Combined \\
\hline Single & $157(56 \pm 4 \%)$ & $43(48 \pm 7 \%)$ & $23(56 \pm 12 \%)$ & $223(54 \pm 4 \%)$ \\
\hline Double & $81(29 \pm 3 \%)$ & $28(31 \pm 6 \%)$ & $16(39 \pm 10 \%)$ & $125(31 \pm 3 \%)$ \\
\hline Triple & $22(8 \pm 2 \%)$ & $9(10 \pm 3 \%)$ & $0(0 \pm 0 \%)$ & $31(8 \pm 1 \%)$ \\
\hline Multiple & $2(1 \pm 1 \%)$ & $4(5 \pm 2 \%)$ & $0(0 \pm 0 \%)$ & $6(1 \pm 1 \%)$ \\
\hline Diffuse & $18(6 \pm 2 \%)$ & $5(6 \pm 3 \%)$ & $2(5 \pm 3 \%)$ & $25(6 \pm 1 \%)$ \\
\hline \# Observed & 280 & 89 & 41 & 410 \\
\hline
\end{tabular}

steeper, as $30 \%$ of the WN sources were not detected in our $4.86 \mathrm{GHz}$ VLA observations, and may therefore have even more steepened high-frequency spectral indices.

\subsection{Radio source properties}

\subsubsection{Radio source structure and angular size}

In Table 5, we give the distribution of the radio structures of the 410 USS sources for which we have good radiomaps. At first sight, all three our samples have basically the same percentage of resolved sources, but the similar value for the MP sample is misleading, as it was observed at much lower resolution.

Our results are different from the USS sample of Röttgering et al. (1994), which contains only $18 \%$ unresolved sources at comparable resolution (1".5). To check if this effect is due to the fainter sources in our sample, we compared our sample with the deep high resolution

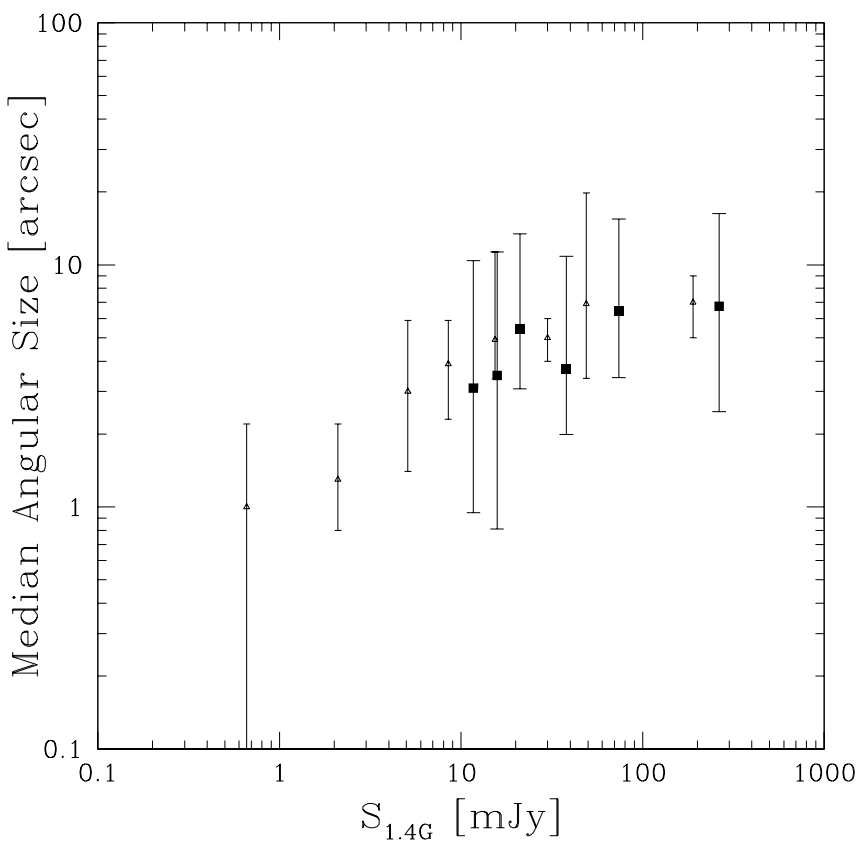

Fig. 10. Median angular size for the flux density limited, spectrally unbiased WSRT samples of Oort 1988 (open triangles), and for our combined USS samples (filled squares). The sources have been binned in equal number bins, and errors represent the $35 \%$ and $65 \%$ levels of the distribution. Note that our USS selection does not affect the value of the median, and that our USS samples also exclude sources that fall below the break at $S_{1400} \lesssim 10 \mathrm{mJy}$

VLA observations of spectrally unbiased sources (Oort 1988; Coleman \& Condon 1985). The resolution of our observations is significantly better than the median angular size for $S_{1400}>1 \mathrm{mJy}$ sources, allowing us to accurately determine the median angular sizes in our samples. We find that our USS sources have a constant median angular size of $\sim 6^{\prime \prime}$ between $10 \mathrm{mJy}$ and $1 \mathrm{Jy}$ (Fig. 10). This is indistinguishable from the results from samples without spectral index selection. It indicates that our USS selection of sources with $\alpha<-1.3$ and $\Theta<1^{\prime}$ does not bias the angular size distribution in the resulting sample. The "downturn" in angular sizes that occurs at $\sim 1 \mathrm{mJy}$ is probably due to a different radio source population, which consist of lower redshift sources in spiral galaxies (see e.g., Coleman \& Condon 1985; Oort et al. 1987; Benn et al. 1993). By selecting only sources with $S_{1400}>10 \mathrm{mJy}$, we have avoided "contamination" of our sample by these foreground sources.

We have searched for further correlations between spectral index or spectral curvatures and angular size or flux density, but found no significant results, except for a trend for more extended sources to have lower than expected $4.86 \mathrm{GHz}$ flux densities, but this effect can be explained by missing flux at large scales in our VLA observations. 


\subsection{Identifications}

\subsubsection{POSS}

We have searched for optical identifications of our USS sources on the digitized POSS-I. We used the likelihood ratio identification criterion as described by e.g. de Ruiter et al. (1977). In short, this criterion compares the probability that a radio and optical source with a certain positional difference are really associated with the probability that this positional difference is due to confusion with a field object (mostly a foreground star), thereby incorporating positional uncertainties in both radio and optical positions. The ratio of these probabilities is expressed as the likelihood ratio $L R$. In the calculation, we have assumed a density of POSS objects $\rho=4$ $10^{-4^{\prime \prime}-2}$, independent of galactic latitude $b$. We have adopted a likelihood ratio cutoff $\mathcal{L}=1.0$, slightly lower than the values used by de Ruiter et al. (1977) and Röttgering et al. (1994). We list sources with $L R>1.0$ for our USS samples in Tables A.4 to A.6. We have included four WN sources (WN J0704+6318, WN J1259+3121, WN J1628+3932 and WN J2313+3842), two TN sources (TN J0510-1838 and TN J1521 + 0741) and four MP sources (MP J0003-3556, MP J1921-5431, MP J1943-4030 and MP J2357-3445) as identifications because both their optical and radio morphologies are diffuse and overlapping, making it impossible to measure a common radio and optical component, while they are very likely to be associated.

Figure 11 shows the identification fraction of USS sources on the POSS $(R \lesssim 20)$. Because the distributions for the WN and TN are very similar, we have combined both samples to calculate the identification fraction. Unlike the results for 4C USS (Tielens et al. 1979; Blumenthal \& Miley 1979), we do not detect a decrease of the identification fraction with steepening spectral index ${ }^{7}$. We interpret the constant $\sim 15 \%$ identification fraction from our sample as a combined population of foreground objects, primarily consisting of clusters (see next section). Our extremely steep spectral index criterion would then select only radio galaxies too distant to be detected on the POSS $(R \gtrsim 20.0)$.

\subsubsection{Literature}

Using the NASA Extragalactic Database (NED), the SIMBAD database and the $\mathrm{W}^{3}$ Browse at the High Energy Astrophysics Science Archive Research Center, we have searched for known optical and X-ray identifications

\footnotetext{
7 In the Westerbork faint USS (Wieringa \& Katgert 1992) or the USS sample from Röttgering et al. (1994), there is also a decrease in the identification fraction, even at limiting magnitudes of $R=22.5$ and $R=23.7$, indicating that this trend continues out to fainter magnitudes and radio fluxes.
}

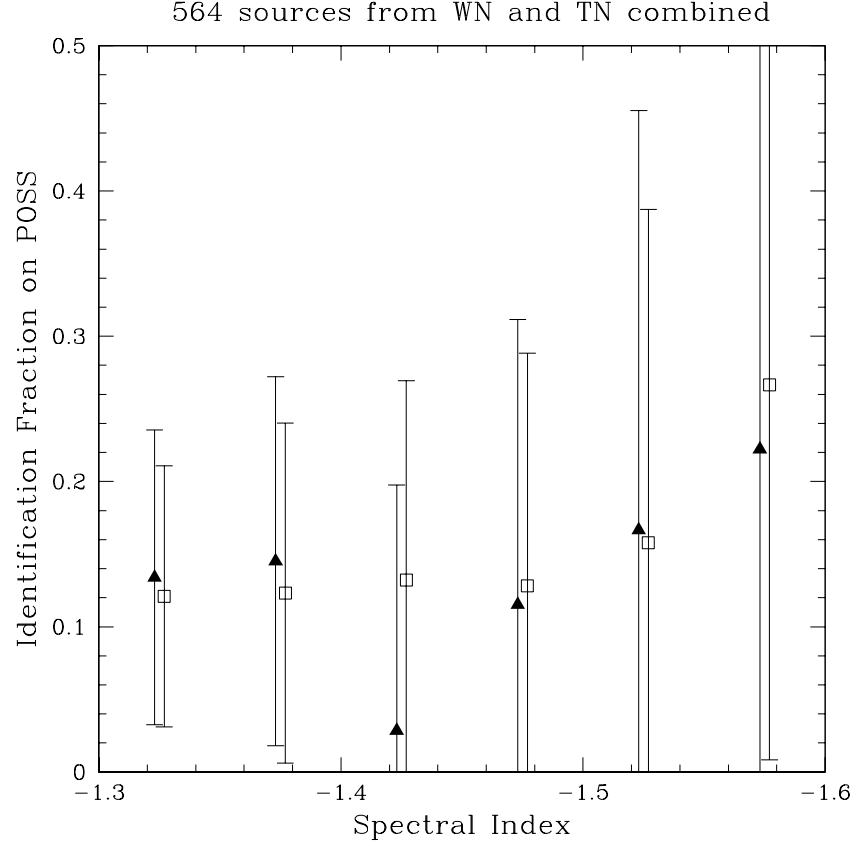

Fig. 11. Identification fraction on the POSS as a function of spectral index for the combined WN and TN sample. Note the absence of a further decrease in the identification fraction with steepening spectral index

of sources in our samples (see Appendices A.7 to A.9). Of the bright optical $(R \lesssim 20)$ identifications, only one source is a known as a K0-star, three (TN J0055+2624, TN J0102-2152, and TN J1521+0742) are "Relic radio galaxies" (Komissarov \& Gubanov 1994; Giovannini et al. 1999), while all others are known galaxy clusters.

All optical cluster identifications, except MP J1943-4030, are also detected in the ROSAT All-Sky survey Bright Source Catalogue (RASS-BSC; Voges et al. 1999). Conversely, of the 23 X-ray sources, seven are known galaxy clusters, and three known galaxies. The remaining 13 sources are good galaxy cluster candidates because they either show a clear over-density of galaxies on the POSS (eight sources), or they have low $\mathrm{X}$-ray count rates $\left(<0.02\right.$ counts $\left.\mathrm{s}^{-1}\right)$, suggesting that these might be more distant galaxy clusters too faint to be detected on the POSS. We conclude that probably $>3 \%$ of our USS sources are associated with galaxy clusters, and that the combined USS + X-ray selection is an efficient (up to $85 \%$ ) selection technique to find galaxy clusters $^{8}$.

Three of our USS sources (WN J2313+4253, TN J0630-2834 and TN J1136+1551) are previously known pulsars (Kaplan et al. 1998). It is worth noting that two out of nine sources in our USS samples with $\alpha<-2$ are known pulsars. Because Lorimer et al. (1995) found the median spectral index of pulsars to

\footnotetext{
8 In the RASS-BSC, only $14 \%$ of the extra-galactic sources are identified with galaxy clusters (Voges et al. 1999).
} 


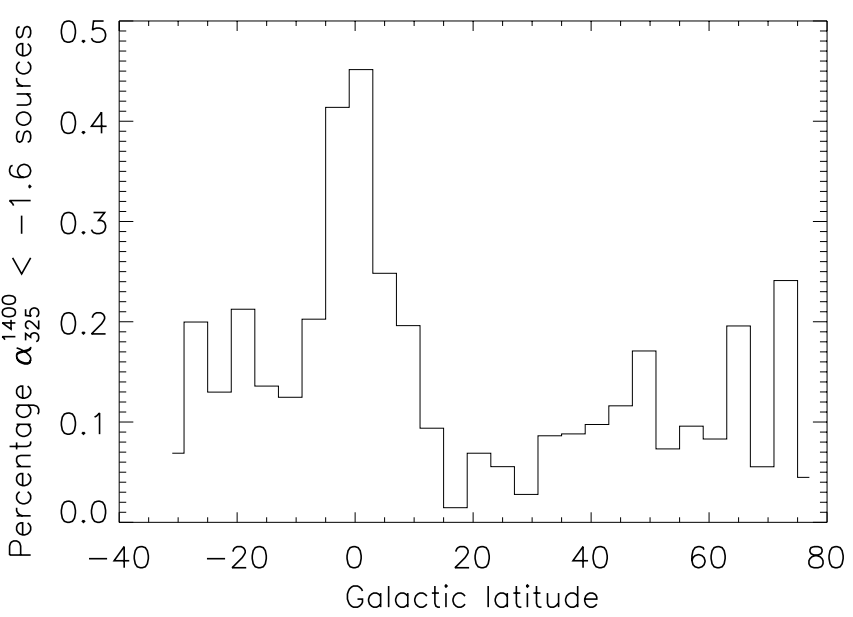

Fig. 12. Percentage of $\alpha_{325}^{1400}<-1.60$ radio sources from a WENSS-NVSS correlation as a function of Galactic latitude. Note the clear peak near the Galactic plane, indicating that these $\alpha_{325}^{1400}<-1.60$ objects might well be Galactic pulsars

be $\sim-1.6$, we examined the distribution of spectral indices as a function of Galactic latitude. In Fig. 12, we plot the percentage of $\alpha_{325}^{1400}<-1.60$ pulsar candidates as a function of Galactic latitude. The four times higher density near the Galactic plane strongly suggests that the majority of these $\alpha_{325}^{1400}<-1.60$ sources are indeed pulsars, which are confined to our Galaxy. A sample of such $\alpha_{325}^{1400}<-1.60$ sources at $|b|<15^{\circ}$ would be an efficient pulsar search method.

We also note that no known quasars are present in our sample. Preliminary results from our optical spectroscopy campaign (De Breuck et al. 1998b, 2000) indicate that $\sim 10 \%$ of our sample are quasars. We interpret this lack of previously known quasars as a selection bias in quasar samples against USS sources.

At $R \gtrsim 20$, all five USS sources with known redshift are HzRGs, indicating a selection of sources without detections on the POSS strongly increases our chances of finding HzRGs.

\section{Conclusions}

We have constructed three spatially separated samples of USS sources containing a total of 669 objects. High-resolution radio observations of more than half of these show that the median size is $\sim 6^{\prime \prime}$, independent of $1.4 \mathrm{GHz}$ flux density, which is consistent with results of similar resolution surveys of samples without spectral index selection. The absence of a downturn in angular size at the lowest fluxes indicates that we do not include significant numbers of spiral galaxies in our sample. A USS sample fainter than ours would therefore include more of these foreground sources, and be less efficient to find HzRGs.

The identification fraction on the POSS is $\sim 15 \%$, with no clear dependence on spectral index, indicating that the HzRGs in the sample are all too distant to be detected, and the POSS detections consist of different classes of objects. A correlation of our USS samples with X-ray catalogs showed that at least $85 \%$ of the X-ray identifications seem to be galaxy clusters known from the literature or by inspection of the POSS. We conclude that (1) the majority of the "non HzRG" USS sources in our sample are clusters, and (2) the combined selection of USS and X-ray sources is an extremely efficient technique to select galaxy clusters.

The above results indicate that up to $85 \%$ of our USS sources might be HzRGs. To identify these objects, we have started an intensive program of $R$ - and $K$-band imaging on 3-10 $\mathrm{m}$ class telescopes. Initial results from optical spectroscopy indicate that $2 / 3$ are indeed $z>2$ radio galaxies (De Breuck et al. 1998a), and $K$-band imaging of optically undetected $(R>25)$ sources (see e.g., van Breugel et al. 1999a) has already lead to the discovery of the first radio galaxy at $z>5$ (van Breugel et al. 1999b).

Acknowledgements. We are grateful for the excellent help provided by the staff of the VLA and ATCA observatories, with special thanks to Chris Carilli and Greg Taylor (NRAO), and Ray Norris and Kate Brooks (ATNF) for help in with observation planning and data reduction. We thank Hien "Napkin" Tran for his comments on the manuscript. The VLA is a facility of the National Radio Astronomy Observatory, which is operated by Associated Universities Inc. under cooperative agreement with the National Science Foundation. The Australia Telescope is funded by the Commonwealth of Australia for operation as a National Facility managed by CSIRO. The authors made use of the database CATS (Verkhodanov et al. 1997) of the Special Astrophysical Observatory, the NASA/IPAC Extragalactic Database (NED) which is operated by the Jet Propulsion Laboratory, California Institute of Technology, under contract with the National Aeronautics and Space Administration, and the High Energy Astrophysics Science Archive Research Center Online Service, provided by the NASA/Goddard Space Flight Center. The work by C.D.B. and W.V.B. was performed under auspices of the U.S. Department of Energy by University of California Lawrence Livermore National Laboratory under contract No. W-7405-Eng-48. 
Appendix A: Source lists

Table A.1. WN sample




Table A.1. continued

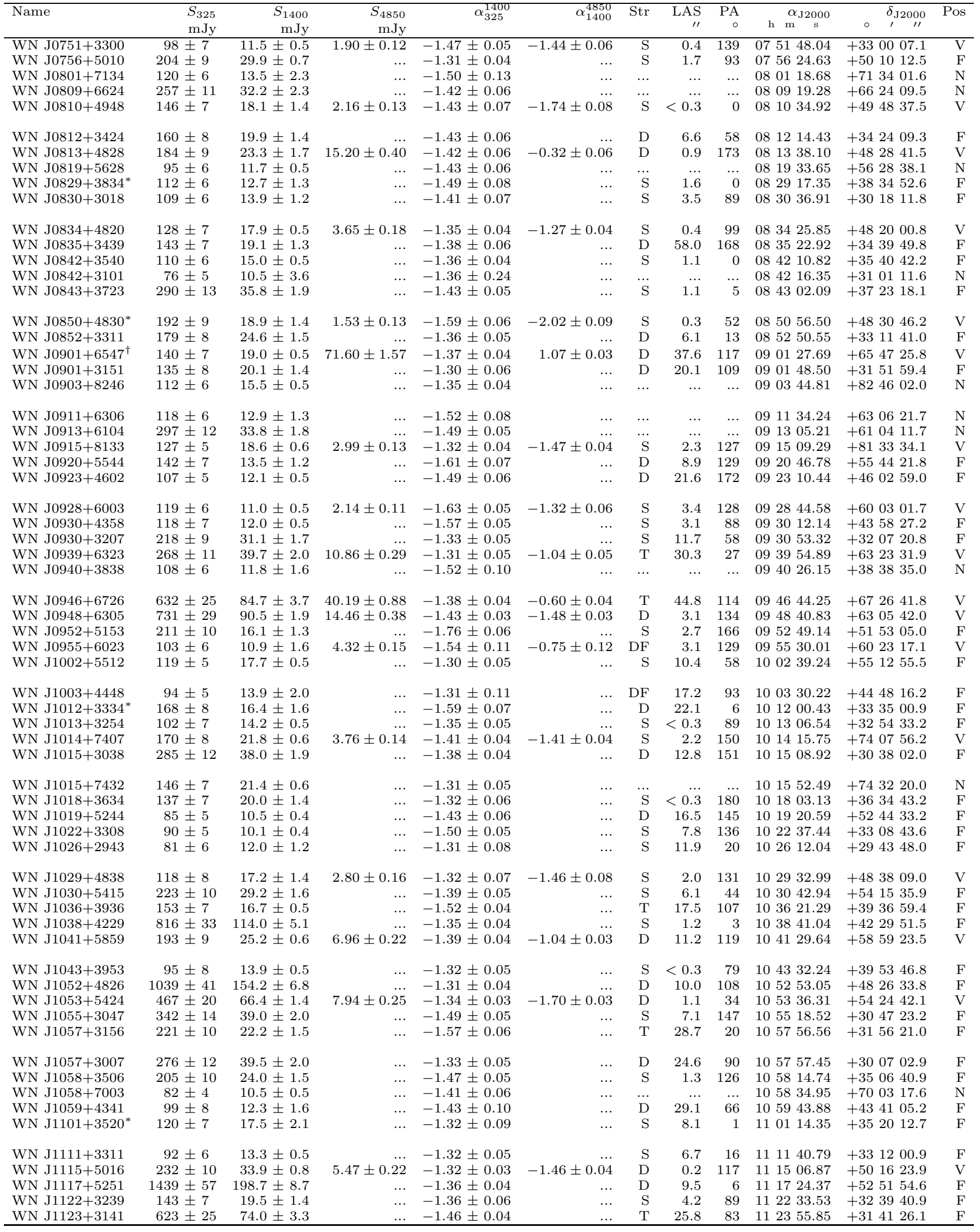


Table A.1. continued

\begin{tabular}{|c|c|c|c|c|c|c|c|c|c|c|c|}
\hline \multirow[t]{2}{*}{ Name } & \multirow{2}{*}{$\begin{array}{l}S_{325} \\
\text { mJy }\end{array}$} & \multirow{2}{*}{$\begin{array}{r}S_{1400} \\
\mathrm{mJy}\end{array}$} & \multirow{2}{*}{$\begin{array}{r}S_{4850} \\
\mathrm{mJy}\end{array}$} & \multirow[t]{2}{*}{$\alpha_{325}^{1400}$} & \multirow[t]{2}{*}{$\alpha_{1400}^{4850}$} & \multirow[t]{2}{*}{ Str } & \multirow{2}{*}{$\begin{array}{r}\mathrm{LAS} \\
\prime \prime\end{array}$} & \multirow{2}{*}{$\begin{array}{c}\mathrm{PA} \\
0\end{array}$} & \multirow{2}{*}{$\begin{array}{l}{ }_{\mathrm{h}} \\
\mathrm{m}_{\mathrm{J} 2000}\end{array}$} & $\delta_{\mathrm{J} 2000}$ & Pos \\
\hline & & & & & & & & & & 111 & \\
\hline WN J1124+3228 & $263 \pm 11$ & $38.9 \pm 0.9$ & $\cdots$ & $-1.31 \pm 0.03$ & $\ldots$ & $\mathrm{S}$ & 5.7 & 165 & 112434.17 & +322819.6 & $\bar{F}$ \\
\hline WN J1126+8318 & $175 \pm 8$ & $25.8 \pm 1.6$ & $\ldots$ & $-1.31 \pm 0.05$ & $\ldots$ & $\ldots$ & $\ldots$ & $\ldots$ & 112626.48 & +831823.8 & $\mathrm{N}$ \\
\hline WN J1128+4822 & $280 \pm 12$ & $29.9 \pm 0.7$ & $\ldots$ & $-1.53 \pm 0.03$ & $\ldots$ & $\mathrm{S}$ & 1.2 & 171 & 112805.19 & +482256.6 & $\mathrm{~F}$ \\
\hline WN J1130+4911 & $134 \pm 6$ & $18.9 \pm 1.5$ & $\ldots$ & $-1.34 \pm 0.07$ & $\ldots$ & $\mathrm{T}$ & 37.0 & 145 & 113017.53 & +491117.7 & $\mathrm{~F}$ \\
\hline WN J1139+3048 & $819 \pm 33$ & $119.6 \pm 5.3$ & $\ldots$ & $-1.32 \pm 0.04$ & $\ldots$ & $\mathrm{D}$ & 9.0 & 40 & 113914.34 & +304850.7 & $\mathrm{~F}$ \\
\hline WN J1139+3706 & $139 \pm 8$ & $16.7 \pm 1.4$ & $\ldots$ & $-1.45 \pm 0.07$ & $\ldots$ & $\mathrm{S}$ & 3.5 & 32 & 113943.04 & +370655.7 & $\mathrm{F}$ \\
\hline WN J1148+5116 & $148 \pm 7$ & $20.5 \pm 1.7$ & $\ldots$ & $-1.35 \pm 0.07$ & $\ldots$ & $\mathrm{D}$ & 6.7 & 50 & $1148 \quad 01.92$ & +51 1629.6 & $\mathrm{~F}$ \\
\hline WN J1148+6233 & $98 \pm 5$ & $14.1 \pm 0.5$ & $1.37 \pm 0.09$ & $-1.33 \pm 0.05$ & $-1.88 \pm 0.06$ & $\mathrm{~S}$ & 2.4 & 144 & 114844.42 & +623324.6 & V \\
\hline WN J1148+3519 & $114 \pm 7$ & $13.9 \pm 1.7$ & $\ldots$ & $-1.44 \pm 0.09$ & $\ldots$ & D & 20.0 & 7 & $1148 \quad 54.29$ & +35 1910.1 & $\mathrm{~F}$ \\
\hline WN J1151+4436 & $78 \pm 5$ & $11.1 \pm 0.5$ & $\ldots$ & $-1.34 \pm 0.06$ & $\ldots$ & $\mathrm{D}$ & 15.1 & 20 & 115116.80 & +443610.0 & $\mathrm{~F}$ \\
\hline WN J1152+3732* & $414 \pm 17$ & $17.1 \pm 1.4$ & $\ldots$ & $-2.18 \pm 0.06$ & $\ldots$ & $\mathrm{S}$ & 15.0 & 119 & 115236.34 & +373243.9 & $\mathrm{~F}$ \\
\hline WN J1154+5415 & $153 \pm 7$ & $19.1 \pm 0.5$ & $1.05 \pm 0.10$ & $-1.42 \pm 0.04$ & $-2.33 \pm 0.08$ & $\mathrm{~S}$ & $<0.3$ & 0 & 115418.97 & +541511.4 & V \\
\hline WN J1203+8350 & $132 \pm 6$ & $18.5 \pm 0.5$ & $\ldots$ & $-1.35 \pm 0.04$ & $\ldots$ & $\ldots$ & $\ldots$ & $\cdots$ & 120330.63 & +835037.1 & $\mathrm{~N}$ \\
\hline WN J1204+5014 & $94 \pm 5$ & $11.3 \pm 1.7$ & $\ldots$ & $-1.45 \pm 0.11$ & $\ldots$ & $\ldots$ & & $\ldots$ & 120431.11 & +501449.8 & $\mathrm{~N}$ \\
\hline WN J1208+4301 & $162 \pm 8$ & $13.9 \pm 1.4$ & $\ldots$ & $-1.68 \pm 0.08$ & $\ldots$ & $\mathrm{T}$ & 8.0 & 27 & $\begin{array}{lll}12 & 08 & 42.77\end{array}$ & +430142.3 & $\mathrm{~F}$ \\
\hline WN J1216+4446 & $191 \pm 8$ & $13.6 \pm 0.5$ & $\ldots$ & $-1.81 \pm 0.04$ & $\ldots$ & $\mathrm{S}$ & 6.1 & 98 & 121646.84 & +444651.3 & $\mathrm{~F}$ \\
\hline WN J1218+3143 & $1906 \pm 76$ & $281.5 \pm 12.4$ & $\ldots$ & $-1.31 \pm 0.04$ & $\ldots$ & $\mathrm{S}$ & 5.6 & 65 & 121831.47 & +314341.7 & $\mathrm{~F}$ \\
\hline WN J1219+4644 & $140 \pm 8$ & $20.9 \pm 0.6$ & $2.84 \pm 0.14$ & $-1.30 \pm 0.04$ & $-1.59 \pm 0.05$ & $\mathrm{~S}$ & 0.2 & 157 & 121931.79 & +464449.6 & V \\
\hline WN J1223+4256 & $116 \pm 6$ & $17.2 \pm 0.5$ & $\ldots$ & $-1.31 \pm 0.04$ & $\ldots$ & $\mathrm{S}$ & 4.3 & 179 & 122311.32 & +425657.3 & $\mathrm{~F}$ \\
\hline WN J1224+4956 & $145 \pm 7$ & $21.4 \pm 1.6$ & $\ldots$ & $-1.31 \pm 0.06$ & $\ldots$ & $\mathrm{T}$ & 39.6 & 66 & 122421.03 & +495649.2 & $\mathrm{~F}$ \\
\hline WN J1224+5436 & $790 \pm 31$ & $69.1 \pm 1.5$ & $6.30 \pm 0.20$ & $-1.67 \pm 0.03$ & $-1.92 \pm 0.03$ & $\mathrm{D}$ & 0.9 & 16 & 122452.35 & +543639.9 & $\mathrm{V}$ \\
\hline WN J1226+4836 & $155 \pm 8$ & $17.0 \pm 0.5$ & $\ldots$ & $-1.51 \pm 0.04$ & $\ldots$ & $\mathrm{S}$ & 4.8 & 115 & 122622.45 & +483639.6 & $\mathrm{~F}$ \\
\hline WN J1232+4621* & $133 \pm 6$ & $19.8 \pm 1.8$ & $\ldots$ & $-1.30 \pm 0.07$ & $\ldots$ & DF & 12.5 & 156 & 123239.79 & +462148.1 & $\mathrm{~F}$ \\
\hline WN J1242+3915 & $549 \pm 22$ & $78.4 \pm 1.7$ & $\ldots$ & $-1.33 \pm 0.03$ & $\ldots$ & D & 11.2 & 51 & 124253.09 & +39 1548.6 & $\mathrm{~F}$ \\
\hline WN J1247+6121 & $103 \pm 5$ & $15.4 \pm 2.4$ & $\ldots$ & $-1.30 \pm 0.11$ & $\ldots$ & $\ldots$ & & $\ldots$ & 124745.31 & +612123.3 & $\mathrm{N}$ \\
\hline WN J1249+4043 & $160 \pm 7$ & $22.8 \pm 1.4$ & $\ldots$ & $-1.33 \pm 0.05$ & $\ldots$ & $\mathrm{D}$ & 37.0 & 80 & 124927.43 & +404349.3 & $\mathrm{~F}$ \\
\hline WN J1258+3212 & $182 \pm 12$ & $19.9 \pm 1.5$ & $\cdots$ & $-1.52 \pm 0.06$ & $\cdots$ & $\mathrm{S}$ & 6.7 & 131 & 125823.62 & +321242.4 & $\mathrm{~F}$ \\
\hline WN J1258+5041 & $230 \pm 11$ & $28.8 \pm 2.0$ & $\ldots$ & $-1.42 \pm 0.06$ & $\ldots$ & $\mathrm{T}$ & 44.9 & 58 & 125850.96 & +504142.3 & $\mathrm{~F}$ \\
\hline WN J1259+3121 & $268 \pm 11$ & $34.0 \pm 1.8$ & $\ldots$ & $-1.41 \pm 0.05$ & $\ldots$ & DF & 20.6 & 1 & 125951.96 & +312105.6 & $\mathrm{~F}$ \\
\hline WN J1300+5311 & $157 \pm 7$ & $19.2 \pm 0.5$ & $2.70 \pm 0.16$ & $-1.44 \pm 0.04$ & $-1.58 \pm 0.05$ & $\mathrm{~S}$ & 0.3 & 7 & 130036.25 & +531152.4 & V \\
\hline WN J1303+5437 & $145 \pm 7$ & $15.4 \pm 1.4$ & $\ldots$ & $-1.54 \pm 0.07$ & $\ldots$ & $\mathrm{D}$ & 16.5 & 88 & 130345.48 & +543728.9 & $\mathrm{~F}$ \\
\hline WN J1306+4726 & $100 \pm 7$ & $13.1 \pm 0.5$ & $\ldots$ & $-1.39 \pm 0.05$ & $\ldots$ & $\mathrm{S}$ & 10.2 & 40 & 130635.99 & +472610.1 & $\mathrm{~F}$ \\
\hline WN J1310+5706 & $112 \pm 6$ & $15.9 \pm 0.5$ & $\ldots$ & $-1.34 \pm 0.04$ & $\ldots$ & $\mathrm{S}$ & 4.2 & 99 & 131022.96 & +570657.9 & $\mathrm{~F}$ \\
\hline WN J1310+3820 & $69 \pm 4$ & $10.3 \pm 1.5$ & $\ldots$ & $-1.30 \pm 0.12$ & $\ldots$ & $\mathrm{S}$ & 4.4 & 18 & 131024.08 & +382044.1 & $\mathrm{~F}$ \\
\hline WN J1312+6646 & $291 \pm 12$ & $41.6 \pm 0.9$ & $8.56 \pm 0.24$ & $-1.33 \pm 0.03$ & $-1.27 \pm 0.03$ & $\mathrm{D}$ & 6.3 & 118 & 131245.59 & +664636.1 & V \\
\hline WN J1314+3649 & $286 \pm 11$ & $36.4 \pm 0.8$ & $\ldots$ & $-1.41 \pm 0.03$ & $\ldots$ & $\mathrm{S}$ & 1.3 & 90 & 131417.86 & +364914.6 & $\mathrm{~F}$ \\
\hline WN J1314+3515* & $169 \pm 7$ & $20.2 \pm 1.7$ & $\ldots$ & $-1.45 \pm 0.07$ & $\ldots$ & $\mathrm{DF}$ & 34.2 & 47 & 131425.00 & +351553.9 & $\mathrm{~F}$ \\
\hline WN J1315+4337 & $149 \pm 8$ & $16.3 \pm 0.5$ & $\ldots$ & $-1.52 \pm 0.04$ & $\ldots$ & S & 1.3 & 152 & 131519.05 & +433757.1 & $\mathrm{~F}$ \\
\hline WN J1321+3311 & $141 \pm 8$ & $19.0 \pm 0.6$ & $\ldots$ & $-1.37 \pm 0.04$ & $\ldots$ & $\mathrm{D}$ & 14.7 & 63 & 132113.55 & +331131.2 & $\mathrm{~F}$ \\
\hline WN J1323+4713 & $106 \pm 5$ & $14.9 \pm 0.5$ & $\ldots$ & $-1.34 \pm 0.04$ & $\ldots$ & $\mathrm{S}$ & 2.5 & 139 & 132308.31 & +471310.7 & $\mathrm{~F}$ \\
\hline WN J1327+5341 & $196 \pm 9$ & $27.4 \pm 0.7$ & $\ldots$ & $-1.35 \pm 0.04$ & & $\mathrm{~S}$ & 0.6 & 162 & $\begin{array}{lll}13 & 27 & 12.77\end{array}$ & +534120.6 & $\mathrm{~F}$ \\
\hline WN J1327+5332 & $163 \pm 7$ & $20.5 \pm 0.6$ & $2.70 \pm 0.17$ & $-1.42 \pm 0.04$ & $-1.61 \pm 0.06$ & $\mathrm{D}$ & 0.3 & 89 & 132737.86 & +533210.8 & $\mathrm{~V}$ \\
\hline WN J1329+3046* & $86 \pm 5$ & $11.7 \pm 1.7$ & $\ldots$ & $-1.37 \pm 0.11$ & $\ldots$ & $\mathrm{S}$ & 1.6 & 6 & 132931.75 & +304613.6 & $\mathrm{~F}$ \\
\hline WN J1329+3046* & $88 \pm 5$ & $12.1 \pm 2.0$ & $\ldots$ & $-1.36 \pm 0.13$ & $\ldots$ & S & 3.6 & 129 & 132955.12 & +304651.2 & $\mathrm{~F}$ \\
\hline WN J1330+6505 & $74 \pm 6$ & $10.5 \pm 0.5$ & $2.34 \pm 0.12$ & $-1.34 \pm 0.06$ & $-1.21 \pm 0.06$ & $\mathrm{~S}$ & 1.8 & 5 & 133002.77 & +650459.3 & V \\
\hline WN J1330+3037* & $496 \pm 20$ & $64.5 \pm 2.9$ & $\ldots$ & $-1.40 \pm 0.04$ & $\cdots$ & D & 25.5 & 140 & 133053.67 & +303759.1 & $\mathrm{~F}$ \\
\hline WN J1330+3604 & $103 \pm 5$ & $15.1 \pm 0.5$ & & $-1.31 \pm 0.05$ & & $\mathrm{D}$ & 9.6 & 0 & 133056.83 & +360401.6 & $\mathrm{~F}$ \\
\hline WN J1330+5344* & $525 \pm 21$ & $71.0 \pm 3.2$ & $8.10 \pm 0.29$ & $-1.37 \pm 0.04$ & $-1.74 \pm 0.05$ & DF & 2.5 & 173 & 133058.90 & +534407.8 & V \\
\hline WN J1331+2937 & $93 \pm 5$ & $12.5 \pm 1.3$ & $3.44 \pm 0.13$ & $-1.37 \pm 0.09$ & $-1.04 \pm 0.09$ & $\mathrm{~S}$ & 2.7 & 40 & 133121.58 & +293712.6 & V \\
\hline WN J1332+3009* & $239 \pm 10$ & $35.3 \pm 2.0$ & $\ldots$ & $-1.31 \pm 0.05$ & $\ldots$ & $\mathrm{D}$ & 39.2 & 161 & 133245.73 & +300959.9 & $\mathrm{~F}$ \\
\hline WN J1333+3037* & $423 \pm 17$ & $21.5 \pm 0.6$ & & $-2.04 \pm 0.03$ & & $\mathrm{~S}$ & 0.4 & 179 & 133321.20 & +303735.1 & $\mathrm{~F}$ \\
\hline WN J1333+4913 & $149 \pm 7$ & $22.2 \pm 0.6$ & $4.78 \pm 0.23$ & $-1.30 \pm 0.04$ & $-1.22 \pm 0.04$ & $\mathrm{~T}$ & 5.4 & 40 & 133356.42 & +491327.1 & V \\
\hline WN J1335+3222* & $150 \pm 7$ & $18.3 \pm 1.4$ & $\ldots$ & $-1.44 \pm 0.06$ & $\ldots$ & D & 8.8 & 85 & 133535.44 & +322248.7 & $\mathrm{~F}$ \\
\hline WN J1336+3820 & $116 \pm 6$ & $15.6 \pm 0.5$ & $\ldots$ & $-1.37 \pm 0.04$ & $\ldots$ & $\mathrm{S}$ & 3.4 & 61 & 133640.88 & +382002.2 & $\mathrm{~F}$ \\
\hline WN J1337+3149 & $142 \pm 7$ & $19.6 \pm 1.9$ & $\ldots$ & $-1.36 \pm 0.08$ & $\cdots$ & $\mathrm{D}$ & 35.2 & 21 & 133702.46 & +314949.2 & $\mathrm{~F}$ \\
\hline WN J1337+3401 & $109 \pm 8$ & $14.3 \pm 0.5$ & $\ldots$ & $-1.39 \pm 0.04$ & & $\mathrm{~S}$ & 3.5 & 56 & 133710.50 & +340126.8 & $\mathrm{~F}$ \\
\hline WN J1339+5320 & $104 \pm 5$ & $13.9 \pm 0.5$ & $1.80 \pm 0.15$ & $-1.38 \pm 0.05$ & $-1.63 \pm 0.07$ & $\mathrm{D}$ & 0.1 & 138 & 133959.25 & +532007.8 & V \\
\hline WN J1341+4953 & $153 \pm 8$ & $16.4 \pm 1.4$ & $\ldots$ & $-1.53 \pm 0.07$ & & $\mathrm{D}$ & 18.6 & 160 & 134125.70 & +495341.8 & $\mathrm{F}$ \\
\hline WN J1346+6736 & $107 \pm 6$ & $14.5 \pm 0.5$ & $2.82 \pm 0.16$ & $-1.37 \pm 0.04$ & $-1.32 \pm 0.05$ & $\mathrm{~S}$ & $<0.3$ & 0 & 134637.02 & +673621.7 & V \\
\hline WN J1347+3033 & $341 \pm 14$ & $42.4 \pm 2.2$ & $\ldots$ & $-1.43 \pm 0.05$ & $\ldots$ & $\mathrm{D}$ & 37.3 & 151 & $1347 \quad 16.09$ & +303307.6 & $\mathrm{~F}$ \\
\hline
\end{tabular}


Table A.1. continued

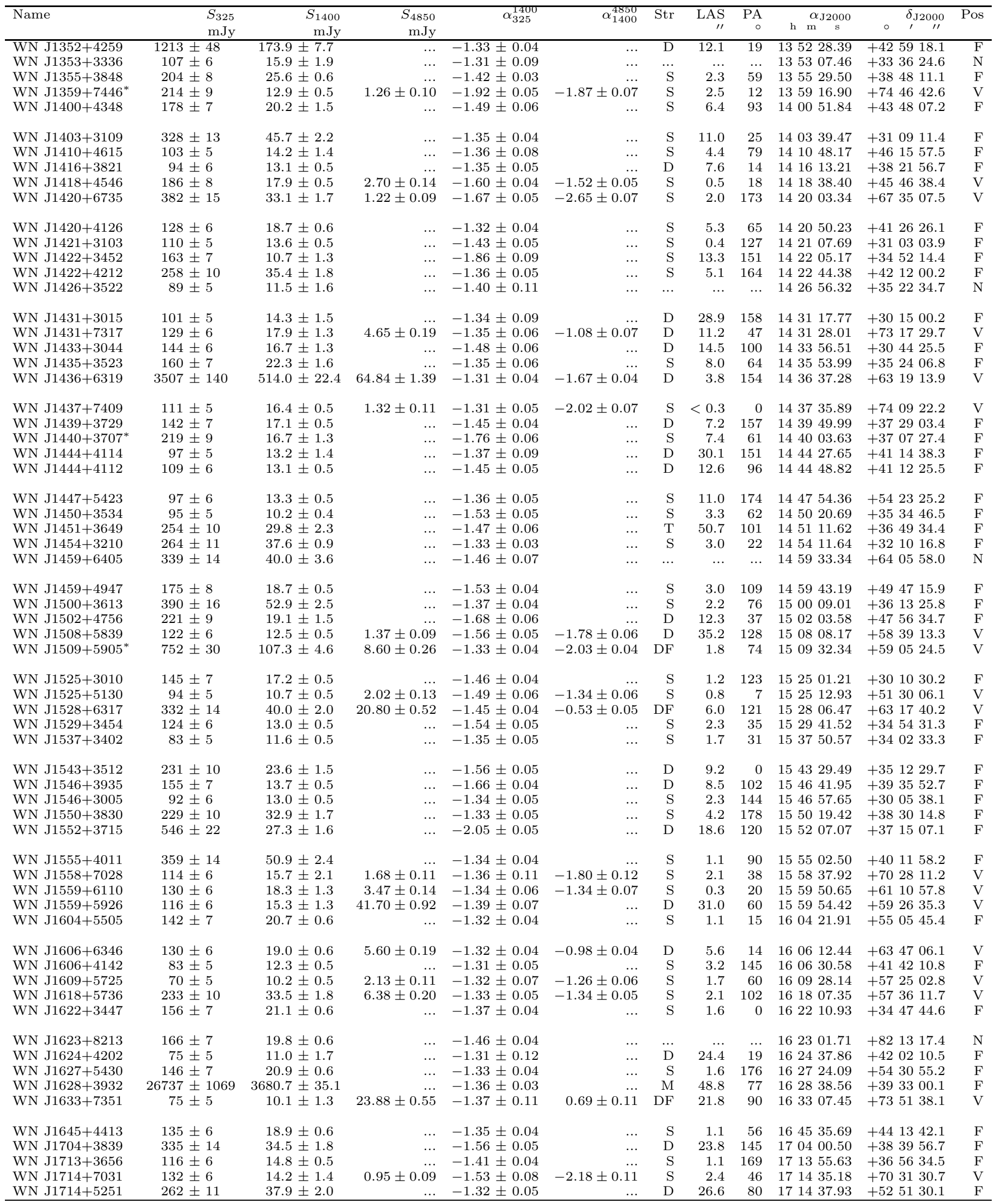


Table A.1. continued

\begin{tabular}{|c|c|c|c|c|c|c|c|c|c|c|c|}
\hline Name & $\begin{array}{l}S_{325} \\
\text { mJy }\end{array}$ & $\begin{array}{r}S_{1400} \\
\mathrm{mJy}\end{array}$ & $\begin{array}{r}S_{4850} \\
\mathrm{mJy}\end{array}$ & $\alpha_{325}^{1400}$ & $\alpha_{1400}^{4850}$ & Str & $\begin{array}{c}\mathrm{LAS}^{\prime \prime} \\
\end{array}$ & $\begin{array}{r}\mathrm{PA} \\
0\end{array}$ & $\mathrm{~h} \underset{\mathrm{m}}{\alpha_{\mathrm{J} 2000}}$ & $\delta_{\mathrm{J} 2000}$ & Pos \\
\hline WN J1717+3828 & $368 \pm 15$ & $35.8 \pm 2.0$ & & $-1.60 \pm 0.05$ & & $\bar{M}$ & 33.6 & 156 & $\begin{array}{llll}17 & 17 & 16.22\end{array}$ & +382816.5 & $\bar{F}$ \\
\hline WN J1718+5823 & $1098 \pm 44$ & $133.2 \pm 2.7$ & $11.26 \pm 0.32$ & $-1.44 \pm 0.03$ & $-1.98 \pm 0.03$ & $\mathrm{~S}$ & 0.3 & 107 & $1718 \quad 18.51$ & +582321.8 & V \\
\hline WN J1723+5822 & $88 \pm 6$ & $10.4 \pm 0.5$ & $2.27 \pm 0.11$ & $-1.46 \pm 0.06$ & $-1.23 \pm 0.06$ & $\mathrm{~S}$ & 4.7 & 103 & 172301.98 & +582244.0 & $\mathrm{~V}$ \\
\hline WN J1731+4640 & $200 \pm 9$ & $15.7 \pm 0.5$ & $\ldots$ & $-1.74 \pm 0.04$ & $\ldots$ & $\mathrm{D}$ & 11.6 & 106 & 173146.10 & +464003.1 & $\mathrm{~F}$ \\
\hline WN J1731+4654 & $323 \pm 13$ & $42.0 \pm 2.1$ & & $-1.40 \pm 0.05$ & & $\mathrm{~S}$ & 1.9 & 176 & 173159.63 & +465400.2 & $\mathrm{F}$ \\
\hline WN J1732+6757 & $85 \pm 5$ & $11.9 \pm 0.5$ & $2.31 \pm 0.11$ & $-1.35 \pm 0.05$ & $-1.32 \pm 0.05$ & $\mathrm{D}$ & 1.5 & 178 & 173230.61 & +675702.5 & V \\
\hline WN J1734+4527 & $87 \pm 5$ & $11.0 \pm 0.5$ & $\ldots$ & $-1.42 \pm 0.06$ & $\ldots$ & $\mathrm{D}$ & 10.4 & 174 & 173407.58 & +452725.6 & $\mathrm{~F}$ \\
\hline WN J1734+3606 & $69 \pm 3$ & $10.2 \pm 3.2$ & $3.12 \pm 0.13$ & $-1.31 \pm 0.22$ & $-0.95 \pm 0.25$ & $\mathrm{~S}$ & 2.1 & 137 & 173424.13 & +360638.8 & V \\
\hline VN J1736+6502 & $193 \pm 8$ & $27.4 \pm 1.6$ & $40 \pm 0.16$ & $-1.34 \pm 0.05$ & $-1.47 \pm 0.06$ & $\mathrm{D}$ & 16.0 & 159 & 173637.50 & +650228.7 & $\mathrm{~V}$ \\
\hline $\mathrm{N} \mathrm{J}$ & $78 \pm 5$ & $11.3 \pm 0.5$ & $\ldots$ & $-1.32 \pm 0.06$ & $\ldots$ & $\mathrm{S}$ & 7.3 & 114 & 173928.39 & +530941.8 & $\mathrm{F}$ \\
\hline N J1749+5 & $124 \pm 7$ & $15.9 \pm 1.6$ & $\ldots$ & $-1.41 \pm 0.08$ & $\cdots$ & $\ldots$ & $\ldots$ & $\ldots$ & 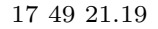 & +565959.6 & $\mathrm{N}$ \\
\hline WN J1749+6248 & $104 \pm 6$ & $13.3 \pm 0.5$ & $2.93 \pm 0.12$ & $-1.41 \pm 0.05$ & $-1.22 \pm 0.05$ & $\mathrm{~S}$ & 1.7 & 48 & 174927.04 & +624854.0 & V \\
\hline WN J1752+2949 & $135 \pm 6$ & $16.9 \pm 0.5$ & $2.29 \pm 0.14$ & $-1.42 \pm 0.04$ & $-1.61 \pm 0.05$ & $\mathrm{~S}$ & 3.2 & 102 & 175256.67 & +294928.3 & V \\
\hline WN J1801+3336 & $136 \pm 6$ & $11.6 \pm 0.5$ & $1.26 \pm 0.12$ & $-1.69 \pm 0.05$ & $-1.78 \pm 0.08$ & $\mathrm{~S}$ & 2.5 & 34 & $\begin{array}{llll}18 & 01 & 07.26\end{array}$ & +333640.4 & $\mathrm{~V}$ \\
\hline WN J1804+5547 & $173 \pm 8$ & $22.9 \pm 1.7$ & & $-1.38 \pm 0.06$ & & 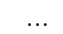 & $\ldots$ & $\ldots$ & 180415.35 & +554716.3 & $\mathrm{~N}$ \\
\hline WN J1804+3048 & $192 \pm 8$ & $27.7 \pm 0.7$ & $5.88 \pm 0.18$ & $-1.33 \pm 0.04$ & $-1.25 \pm 0.03$ & $\mathrm{~S}$ & 2.0 & 84 & 180442.01 & +304845.0 & V \\
\hline WN J1806+6332 & $138 \pm 7$ & $17.7 \pm 0.5$ & $\ldots$ & $-1.41 \pm 0.04$ & $\ldots$ & $\ldots$ & $\ldots$ & $\ldots$ & 180623.66 & +633207.9 & $\mathrm{~N}$ \\
\hline VN J18 & $483 \pm 19$ & $71.4 \pm 3.3$ & $67 \pm 0.31$ & $1.31 \pm 0.04$ & $16 \pm 0.04$ & $\mathrm{D}$ & 3.7 & 173 & $\begin{array}{lll}18 & 07 & 07.39\end{array}$ & +502725.8 & V \\
\hline WN J & $76 \pm 5$ & $10.9 \pm 1.4$ & \pm 0.11 & $3 \pm 0.10$ & $8 \pm 0.13$ & $\mathrm{~S}$ & 0.3 & 103 & 180729.75 & +662833.4 & V \\
\hline WN J181 & $115 \pm 8$ & $14.1 \pm 0.5$ & $2 \pm 0.12$ & $-1.44 \pm 0.04$ & $-1.36 \pm 0.05$ & $\mathrm{~S}$ & 1.6 & 15 & $\begin{array}{lll}18 & 10 & 28.41\end{array}$ & +663515.1 & V \\
\hline WN J1811+6008 & $160 \pm 7$ & $17.8 \pm 0.5$ & $7.87 \pm 0.24$ & $-1.50 \pm 0.04$ & $-0.66 \pm 0.03$ & $\mathrm{DF}$ & 3.5 & 31 & 181118.38 & +600842.9 & V \\
\hline WN J1813+4847 & $92 \pm 5$ & $12.1 \pm 0.5$ & $\ldots$ & $-1.39 \pm 0.06$ & $\ldots$ & $\ldots$ & $\ldots$ & $\ldots$ & 181316.68 & +484750.1 & $\mathrm{~N}$ \\
\hline VN J1814+5009 & $120 \pm 7$ & $16.7 \pm 0.5$ & & $-1.35 \pm 0.05$ & & $\ldots$ & $\ldots$ & $\ldots$ & 181401.40 & +500958.0 & $\mathrm{~N}$ \\
\hline WN J & $4 \pm 6$ & $17.6 \pm 0.6$ & $2.96 \pm 0.14$ & $-1.34 \pm 0.04$ & $-1.43 \pm 0.05$ & $\mathrm{~S}$ & 1.6 & 48 & $18 \quad 15 \quad 23.11$ & +365600.9 & V \\
\hline WN J1 & $6 \pm 8$ & $27.3 \pm 1.6$ & & $-1.31 \pm 0.05$ & & $\ldots$ & $\ldots$ & $\ldots$ & $1816 \quad 26.11$ & +384053.0 & $\mathrm{~N}$ \\
\hline $3+7042$ & $1624 \pm 65$ & $199.6 \pm 8.8$ & $30.50 \pm 0.74$ & $-1.44 \pm 0.04$ & $-1.51 \pm 0.04$ & $\mathrm{D}$ & 1.4 & 10 & 181804.24 & +704259.5 & V \\
\hline WN J1818+3852 & $105 \pm 6$ & $12.6 \pm 0.5$ & $1.29 \pm 0.09$ & $-1.45 \pm 0.05$ & $-1.84 \pm 0.07$ & $\mathrm{~S}$ & 2.6 & 64 & 181842.17 & +385220.4 & V \\
\hline $\mathrm{VN} \mathrm{J}$ & $7 \pm 7$ & $3.7 \pm 0.6$ & \pm 0.19 & $4 \pm 0.04$ & \pm 0.03 & $\mathrm{~T}$ & 7.5 & 103 & $18 \quad 18 \quad 47.90$ & +342824.0 & $\mathrm{~V}$ \\
\hline WN J1 & $149 \pm 6$ & $1.9 \pm 1.5$ & $.11 \pm 0.10$ & $-1.31 \pm 0.06$ & $-2.40 \pm 0.09$ & $\mathrm{~S}$ & 1.6 & 11 & $1818 \quad 50.55$ & +614418.4 & $\mathrm{~V}$ \\
\hline WN J181 & $77 \pm 5$ & $0.5 \pm 0.5$ & & $-1.36 \pm 0.06$ & & $\ldots$ & $\ldots$ & $\ldots$ & 181928.60 & +312219.3 & $\mathrm{~N}$ \\
\hline WN J1821+3602 & $398 \pm 136$ & $9.0 \pm 4.2$ & $33.33 \pm 4.90$ & $-2.42 \pm 0.04$ & $0.69 \pm 0.04$ & $\mathrm{~T}$ & 35.0 & 55 & 182102.20 & +360215.7 & V \\
\hline 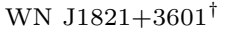 & $51 \pm 6$ & $0.2 \pm 1.5$ & \pm 2.53 & $5 \pm 0.11$ & $88 \pm 0.12$ & $\mathrm{D}$ & 34.9 & 55 & 182122.59 & +360103.3 & V \\
\hline WN J1829+6914 & $207 \pm 9$ & $3.0 \pm 1.3$ & $5 \pm 0.12$ & $-1.90 \pm 0.08$ & $-1.41 \pm 0.09$ & $\mathrm{~S}$ & 1.8 & 22 & 182905.68 & +691406.1 & V \\
\hline WN J1829+4919 & $98 \pm 6$ & $14.2 \pm 0.5$ & $7.95 \pm 0.24$ & $-1.32 \pm 0.06$ & $-0.47 \pm 0.04$ & $\mathrm{D}$ & 12.5 & 173 & 182933.74 & +491955.6 & V \\
\hline WN J1829+5945 & $346 \pm 14$ & $50.4 \pm 2.4$ & $8.82 \pm 0.25$ & $-1.32 \pm 0.04$ & $-1.40 \pm 0.04$ & $\mathrm{~S}$ & 1.7 & 26 & 182957.26 & +594503.2 & V \\
\hline VN J1830+6422 & $70 \pm 3$ & $10.3 \pm 0.5$ & $\ldots$ & $-1.31 \pm 0.07$ & $\ldots$ & $\ldots$ & $\ldots$ & $\ldots$ & 183045.82 & +642226.3 & $\mathrm{~N}$ \\
\hline WN J18: & $6 \pm 10$ & $8.7 \pm 1.6$ & $3.82 \pm 0.59$ & $1 \pm 0.05$ & $-0.15 \pm 0.05$ & $\mathrm{D}$ & 19.4 & 56 & 183241.16 & +535434.0 & V \\
\hline VN J183 & $1 \pm 9$ & $4.2 \pm 1.5$ & $3.54 \pm 0.15$ & $-1.41 \pm 0.05$ & $-1.55 \pm 0.06$ & $\mathrm{~S}$ & 1.4 & 10 & 183623.22 & +521028.4 & V \\
\hline WN J18: & $6 \pm 8$ & $22.8 \pm 1.6$ & & $-1.40 \pm 0.06$ & & $\ldots$ & $\ldots$ & $\ldots$ & 183914.54 & +471059.4 & $\mathrm{~N}$ \\
\hline WN J1843+5932 & $998 \pm 40$ & $148.2 \pm 6.6$ & $30.87 \pm 0.70$ & $-1.31 \pm 0.04$ & $-1.26 \pm 0.04$ & $\mathrm{~T}$ & 7.7 & 64 & 184331.70 & +593259.3 & V \\
\hline $\mathrm{VN}$ & \pm 6 & $.8 \pm 1.3$ & $67 \pm 0.23$ & $2 \pm 0.07$ & $0.69 \pm 0.07$ & DF & 2.9 & 67 & 1847 & +542306.6 & V \\
\hline $\mathrm{N} \mathrm{J}$ & \pm 16 & $4 \pm 2.5$ & $\ldots$ & $6 \pm 0.04$ & $\ldots$ & $\ldots$ & $\ldots$ & $\ldots$ & 18520 & +571 & $\mathrm{~N}$ \\
\hline N J185 & \pm 7 & $.9 \pm 0.6$ & $\ldots$ & $-1.43 \pm 0.04$ & & $\ldots$ & $\ldots$ & $\ldots$ & 185703.60 & +741131.2 & $\mathrm{~N}$ \\
\hline VN J1859+5900 & \pm 6 & $.7 \pm 0.5$ & \pm 0.12 & $-1.46 \pm 0.06$ & $6 \pm \pm 0.07$ & $\mathrm{~S}$ & 0.5 & 171 & 185940.34 & +590036.8 & V \\
\hline WN J1859+5416 & $81 \pm 4$ & $10.1 \pm 1.6$ & $2.70 \pm 0.14$ & $-1.43 \pm 0.12$ & $-1.06 \pm 0.13$ & $\mathrm{~S}$ & 1.7 & 17 & 185957.65 & +541621.8 & V \\
\hline $\mathrm{N} \mathrm{J1}$ & \pm 4 & $0.2 \pm 0.4$ & & $1 \pm 0.05$ & & & $\cdots$ & & 190726 . & +853235.2 & $\mathrm{~N}$ \\
\hline IN J191 & $5 \pm 10$ & $3 \pm 1.5$ & $37 \pm 0.14$ & $2 \pm 0.05$ & $-1.56 \pm 0.06$ & S & 1.8 & 7 & 191149.54 & +634209.6 & V \\
\hline VN J1912+8627 & $6 \pm 7$ & $.8 \pm 1.2$ & $5 \pm 0.13$ & $-1.30 \pm 0.05$ & $-1.61 \pm 0.07$ & $\mathrm{~S}$ & 2.4 & 178 & 191206.26 & +8627 10.1 & V \\
\hline N & $75 \pm 5$ & $.2 \pm 0.5$ & $.37 \pm 0.12$ & $-1.30 \pm 0.06$ & $-1.25 \pm 0.06$ & $\mathrm{~S}$ & 1.9 & 152 & 191735.50 & +663538.5 & V \\
\hline WN J1917+7149 & $280 \pm 12$ & $40.4 \pm 2.1$ & & $-1.33 \pm 0.05$ & $\ldots$ & $\ldots$ & $\ldots$ & $\ldots$ & 191756.11 & +714919.7 & $\mathrm{~N}$ \\
\hline $\mathrm{N}$ & \pm 5 & & & & 05 & $\mathrm{~S}$ & 2.4 & 91 & 192333.18 & +604756.8 & $\mathrm{~V}$ \\
\hline T. & \pm 9 & $7-7$ & $=0.16$ & \pm 0 & .06 & $\mathrm{D}$ & 8.6 & 52 & 192516.97 & +520334.4 & $\mathrm{~V}$ \\
\hline $\mathrm{VN} \mathrm{J}$ & $0 \pm 6$ & $.5 \pm 0.5$ & $3 \pm 0.11$ & $2 \pm 0$. & $8 \pm 0.06$ & $\mathrm{~S}$ & 2.0 & 9 & 192522.22 & +574227.3 & $\mathrm{~V}$ \\
\hline WN J1926+5710 & 367 & $20.2 \pm 0.6$ & $5 \pm 0.18$ & $-1.31 \pm 0.04$ & $-1.20 \pm 0.04$ & $\mathrm{~S}$ & 1.8 & 161 & 192643.85 & +571000.5 & V \\
\hline WN J1927+6436 & $661 \pm 26$ & $80.3 \pm 3.5$ & $6.62 \pm 0.21$ & $-1.44 \pm 0.04$ & $-2.01 \pm 0.04$ & $\mathrm{D}$ & 20.1 & 120 & 192722.83 & +643602.6 & V \\
\hline NN J194 & $367 \pm 55$ & $7.2 \pm 6.5$ & $5 \pm 0.47$ & $-1.53 \pm 0.04$ & $3 \pm 0.04$ & $\mathrm{~S}$ & 1.8 & 165 & 194423.99 & +655223.8 & $\mathrm{~V}$ \\
\hline WN J1953+7052 & $75 \pm 7$ & $10.3 \pm 0.5$ & $3.22 \pm 0.15$ & $-1.36 \pm 0.07$ & $-0.94 \pm 0.05$ & $\mathrm{D}$ & 14.4 & 150 & 195321.10 & +705219.8 & V \\
\hline WN J1954+7011 & $460 \pm 19$ & $63.4 \pm 2.9$ & & $-1.36 \pm 0.04$ & & $\ldots$ & $\ldots$ & $\ldots$ & 195430.53 & +701133.5 & $\mathrm{~N}$ \\
\hline WN J2044+7044 & $311 \pm 13$ & $41.7 \pm 0.9$ & $7.18 \pm 0.22$ & $-1.38 \pm 0.03$ & $-1.42 \pm 0.03$ & D & 1.3 & 83 & 204457.80 & +704403.8 & V \\
\hline WN J2052+6925 & $113 \pm 6$ & $16.1 \pm 1.3$ & $\ldots$ & $-1.33 \pm 0.07$ & $\ldots$ & $\ldots$ & $\ldots$ & $\ldots$ & 205233.78 & +692509.3 & $\mathrm{N}$ \\
\hline
\end{tabular}


Table A.1. continued

\begin{tabular}{|c|c|c|c|c|c|c|c|c|c|c|c|c|}
\hline Name & $\begin{array}{l}S_{325} \\
\text { mJy }\end{array}$ & $\begin{array}{r}S_{1400} \\
\text { mJy }\end{array}$ & $\begin{array}{r}S_{4850} \\
\mathrm{mJy}\end{array}$ & $\alpha_{325}^{1400}$ & $\alpha_{1400}^{4850}$ & Str & $\begin{array}{r}{ }^{\prime \prime} \\
\end{array}$ & $\begin{array}{r}\mathrm{PA} \\
0\end{array}$ & $\mathrm{~h} \underset{\mathrm{m}}{\alpha} \underset{\mathrm{s}}{\alpha}$ & $\circ$ & $\delta_{\mathrm{J} 2000}$ & Pos \\
\hline WN J2053+6849 & $109 \pm 5$ & $15.8 \pm 0.5$ & $2.59 \pm 0.13$ & $-1.32 \pm 0.05$ & $-1.45 \pm 0.05$ & $\bar{S}$ & 2.8 & 13 & 205338.68 & +68 & 4854.7 & $\overline{\mathrm{V}}$ \\
\hline WN J2139+3125 & $249 \pm 10$ & $28.0 \pm 0.7$ & $\ldots$ & $-1.50 \pm 0.04$ & $\ldots$ & $\ldots$ & $\ldots$ & $\ldots$ & 213932.31 & +31 & 2518.7 & $\mathrm{~N}$ \\
\hline WN J2146+3330 & $105 \pm 6$ & $10.8 \pm 0.5$ & $\ldots$ & $-1.56 \pm 0.05$ & $\ldots$ & $\ldots$ & $\ldots$ & $\ldots$ & 214655.47 & +33 & 3001.7 & $\mathrm{~N}$ \\
\hline WN J2147+3137 & $215 \pm 9$ & $25.4 \pm 1.6$ & $\ldots$ & $-1.46 \pm 0.05$ & $\ldots$ & $\ldots$ & $\ldots$ & $\ldots$ & 214735.21 & +31 & 3758.9 & $\mathrm{~N}$ \\
\hline WN J2158+3424 & $182 \pm 8$ & $25.3 \pm 0.7$ & $4.48 \pm 0.17$ & $-1.35 \pm 0.04$ & $-1.39 \pm 0.04$ & $\mathrm{~T}$ & 7.9 & 163 & 215854.14 & +34 & 2447.8 & V \\
\hline WN J2213+3411 & $320 \pm 13$ & $29.8 \pm 2.2$ & $\ldots$ & $-1.63 \pm 0.06$ & $\ldots$ & $\ldots$ & $\ldots$ & $\ldots$ & $\begin{array}{lll}22 & 13 & 12.48\end{array}$ & +34 & 1142.8 & $\mathrm{~N}$ \\
\hline WN J2219+2951 & $151 \pm 10$ & $13.2 \pm 0.5$ & $\ldots$ & $-1.67 \pm 0.04$ & $\ldots$ & $\ldots$ & $\ldots$ & $\ldots$ & 221928.65 & +29 & 5157.4 & $\mathrm{~N}$ \\
\hline WN J2221+3800 & $99 \pm 6$ & $14.4 \pm 0.5$ & $2.18 \pm 0.11$ & $-1.32 \pm 0.05$ & $-1.52 \pm 0.05$ & $\mathrm{~S}$ & 1.4 & 5 & 222149.53 & +38 & 0038.4 & V \\
\hline WN J2222+3305 & $232 \pm 10$ & $30.4 \pm 1.7$ & $3.53 \pm 0.14$ & $-1.39 \pm 0.05$ & $-1.73 \pm 0.05$ & $\mathrm{~S}$ & 1.4 & 13 & $22 \quad 22 \quad 15.19$ & +33 & 0544.1 & V \\
\hline WN J2245+3937 & $204 \pm 8$ & $15.5 \pm 1.3$ & $5.65 \pm 0.21$ & $-1.76 \pm 0.07$ & $-0.81 \pm 0.07$ & DF & 4.0 & 168 & $22 \quad 4502.74$ & +39 & 3727.9 & V \\
\hline WN J2250+4131 & $128 \pm 7$ & $15.2 \pm 1.4$ & $2.03 \pm 0.11$ & $-1.46 \pm 0.07$ & $-1.62 \pm 0.09$ & $\mathrm{~S}$ & 1.5 & 25 & 225051.16 & $+41:$ & 3116.4 & $\mathrm{~V}$ \\
\hline WN J2313+4053 & $101 \pm 6$ & $11.3 \pm 1.4$ & & $-1.50 \pm 0.09$ & & $\ldots$ & $\ldots$ & $\ldots$ & 231306.34 & +40 & 5340.6 & $\mathrm{~N}$ \\
\hline WN J2313+4253 & $94 \pm 7$ & $13.4 \pm 1.5$ & $3.41 \pm 0.14$ & $-1.33 \pm 0.09$ & $-1.10 \pm 0.10$ & $\mathrm{~S}$ & 1.4 & 13 & 231308.62 & +42 & 5313.0 & $\mathrm{~V}$ \\
\hline WN J2313+3842* & $304 \pm 13$ & $13.2 \pm 2.6$ & & $-2.15 \pm 0.14$ & & $\ldots$ & 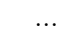 & $\ldots$ & 231347.75 & +38 & 4226.2 & $\mathrm{~N}$ \\
\hline WN J2319+4251 & $321 \pm 14$ & $40.2 \pm 2.9$ & $3.88 \pm 0.14$ & $-1.42 \pm 0.06$ & $-1.88 \pm 0.07$ & $\mathrm{~S}$ & 1.5 & 20 & 231947.25 & +42 & $\begin{array}{lll}51 & 09.2\end{array}$ & V \\
\hline WN J2337+3421 & $84 \pm 4$ & $12.4 \pm 1.3$ & & $-1.31 \pm 0.09$ & & & & & 233723.43 & +34 & 2151.5 & $\mathrm{~N}$ \\
\hline WN J2338+4047 & $115 \pm 6$ & $16.9 \pm 0.5$ & $5.06 \pm 0.18$ & $-1.31 \pm 0.04$ & $-0.97 \pm 0.04$ & $\mathrm{D}$ & 15.7 & 8 & 233811.10 & +40 & 4719.6 & V \\
\hline WN J2350+3631 & $196 \pm 8$ & $22.8 \pm 0.6$ & $2.20 \pm 0.11$ & $-1.47 \pm 0.04$ & $-1.88 \pm 0.04$ & DF & 2.7 & 165 & 235025.69 & +36 & 3127.6 & $\mathrm{~V}$ \\
\hline
\end{tabular}

$\dagger$ Not a real USS source; see notes.

* See notes.

Table A.2. TN sample

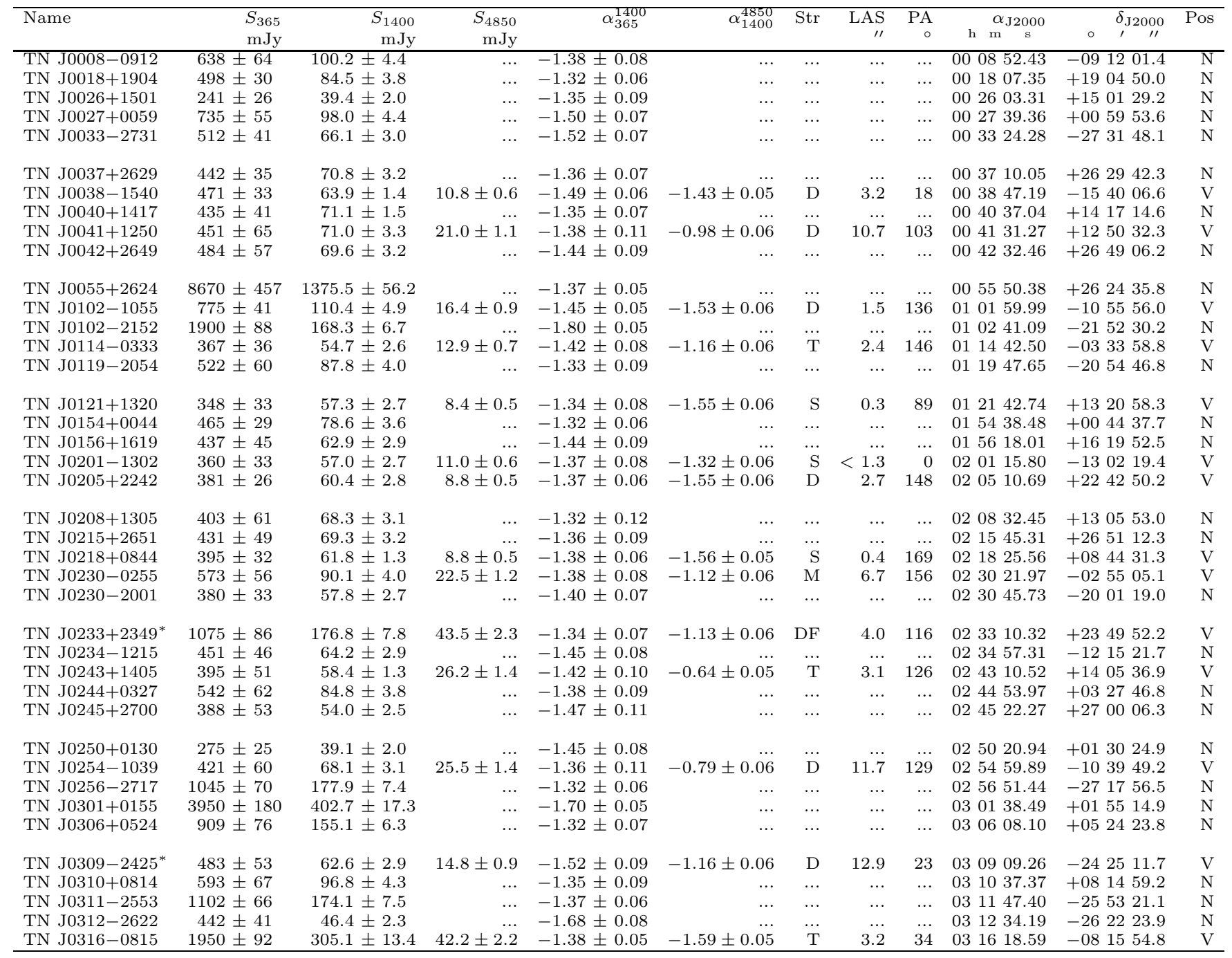


Table A.2. continued

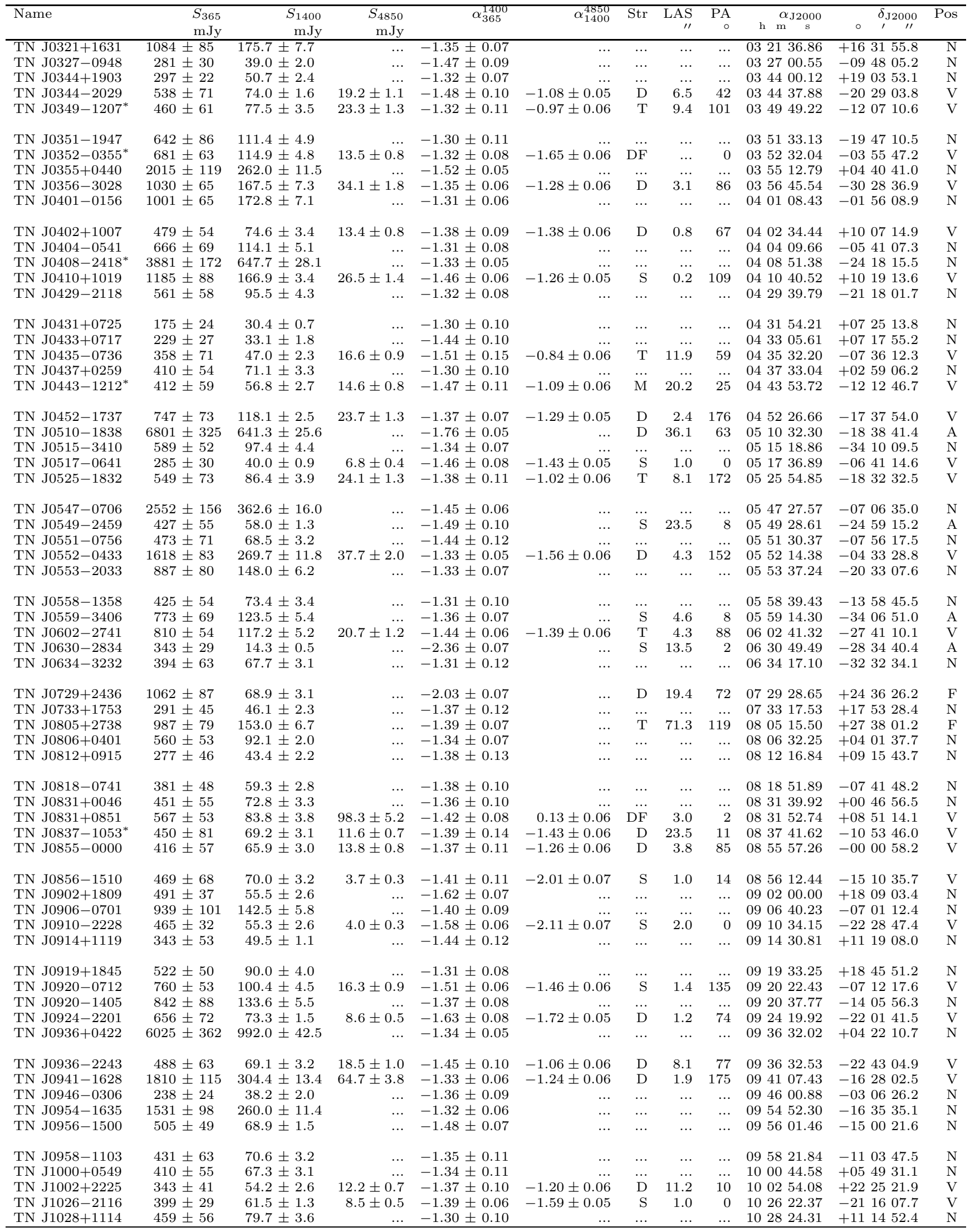


Table A.2. continued

\begin{tabular}{|c|c|c|c|c|c|c|c|c|c|c|c|}
\hline Name & $\begin{array}{l}S_{365} \\
\mathrm{mJy} \\
\end{array}$ & $\begin{array}{r}S_{1400} \\
\mathrm{mJy} \\
\end{array}$ & $\begin{array}{r}S_{4850} \\
\mathrm{mJy}\end{array}$ & $\alpha_{365}^{1400}$ & $\alpha_{1400}^{4850}$ & Str & $\begin{array}{l}\mathrm{LAS}^{\prime \prime} \\
\end{array}$ & $\begin{array}{r}\mathrm{PA} \\
0\end{array}$ & $\begin{array}{cc} & \alpha_{J 2000} \\
h & m \\
\end{array}$ & 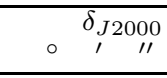 & Pos \\
\hline TN J1031+0259 & $416 \pm 62$ & $60.0 \pm 1.3$ & & $-1.44 \pm 0.11$ & & & & & 103113.36 & +025905.3 & $\bar{N}$ \\
\hline TN J1033-1339 & $981 \pm 75$ & $153.8 \pm 6.8$ & $6.0 \pm 1.4$ & $-1.38 \pm 0.07$ & $-1.43 \pm 0.06$ & $\mathrm{~S}$ & 2.0 & 107 & $1033 \quad 10.70$ & -133952.0 & $\mathrm{~V}$ \\
\hline $1043-1718$ & $578 \pm 70$ & $91.0 \pm 3.9$ & $.9 \pm 1.0$ & $-1.38 \pm 0.10$ & $-1.26 \pm 0.06$ & $\mathrm{D}$ & 34.7 & 63 & $1043 \quad 19.42$ & -171853.5 & V \\
\hline TN J1043+2404 & $439 \pm 34$ & $52.0 \pm 2.5$ & $\ldots$ & $-1.59 \pm 0.07$ & $\ldots$ & $\mathrm{S}$ & 3.7 & 90 & 104343.28 & +240447.3 & $\mathrm{~F}$ \\
\hline TN J1045+1832 & $348 \pm 27$ & $53.6 \pm 1.2$ & $\ldots$ & $-1.39 \pm 0.06$ & ... & $\ldots$ & $\ldots$ & $\ldots$ & 104542.93 & +183237.5 & $\mathrm{N}$ \\
\hline TN J1049-1258 & $818 \pm 56$ & $112.2 \pm 5.0$ & $2 \pm 0.9$ & $-1.48 \pm 0.06$ & $-1.50 \pm 0.06$ & $\mathrm{M}$ & 10.1 & 104 & 104906.22 & -125818.3 & V \\
\hline TN J & $383 \pm 62$ & $63.7 \pm 2.9$ & $\ldots$ & $-1.33 \pm 0.13$ & $\ldots$ & $\ldots$ & $\ldots$ & $\ldots$ & 105344.52 & -151829.8 & $\mathrm{~N}$ \\
\hline TN J1056-0 & $449 \pm 57$ & $57.2 \pm 2.7$ & $\ldots$ & $-1.53 \pm 0.10$ & $\ldots$ & $\ldots$ & $\ldots$ & $\ldots$ & 105652.64 & -040015.9 & $\mathrm{~N}$ \\
\hline TN J1102+1029 & $3713 \pm 224$ & $633.6 \pm 27.8$ & & $-1.32 \pm 0.06$ & $\ldots$ & & & $\ldots$ & 110217.47 & +102908.0 & $\mathrm{N}$ \\
\hline TN J1102-1651 & $688 \pm 68$ & $112.5 \pm 5.0$ & $20.2 \pm 1.1$ & $-1.35 \pm 0.08$ & $-1.38 \pm 0.06$ & $\mathrm{D}$ & 3.0 & 70 & 110247.13 & -165134.4 & $\mathrm{~V}$ \\
\hline TN J1112 & $663 \pm 51$ & $101.0 \pm 4.5$ & $6 \pm 0.9$ & $-1.40 \pm 0.07$ & $-1.45 \pm 0.06$ & $\mathrm{D}$ & 9.1 & 119 & 111223.86 & -294806.4 & $\mathrm{~V}$ \\
\hline TN J & $36 \pm 53$ & $64.9 \pm 3.0$ & $\ldots$ & $-1.33 \pm 0.11$ & $\ldots$ & $\ldots$ & $\ldots$ & $\ldots$ & 111404.82 & -222126.6 & $\mathrm{~N}$ \\
\hline TN J1117-1409 & $785 \pm 69$ & $134.5 \pm 5.9$ & $\ldots$ & $-1.31 \pm 0.07$ & $\ldots$ & $\ldots$ & $\ldots$ & $\ldots$ & 111723.00 & -140909.5 & $\mathrm{~N}$ \\
\hline TN J & $14 \pm 44$ & $.3 \pm 2.9$ & $\ldots$ & $-1.57 \pm 0.07$ & $\ldots$ & & $\ldots$ & $\ldots$ & 111854.08 & -261220.6 & $\mathrm{~N}$ \\
\hline TN J1121-1155 & $32 \pm 65$ & $50.1 \pm 2.4$ & ... & $-1.41 \pm 0.15$ & ... & $\ldots$ & $\ldots$ & $\ldots$ & 112132.75 & $\begin{array}{lll}-11 & 55 & 03.2\end{array}$ & $\mathrm{~N}$ \\
\hline $\mathrm{TN} \mathrm{J}$ & $56 \pm 83$ & $196.8 \pm 8.4$ & & $-1.32 \pm 0.06$ & $\ldots$ & & & $\ldots$ & 112139.89 & +170614.8 & $\mathrm{N}$ \\
\hline TN J & $7 \pm 64$ & $50.6 \pm 2.4$ & $8.5 \pm 0.5$ & $-1.55 \pm 0.12$ & $-1.43 \pm 0.06$ & $\mathrm{~S}$ & 0.8 & 29 & 1123 & -215405.3 & V \\
\hline TN J & $354 \pm 52$ & $61.4 \pm 2.9$ & $\ldots$ & $-1.30 \pm 0.11$ & $\ldots$ & $\ldots$ & $\ldots$ & $\ldots$ & 11255 & -034204.0 & $\mathrm{~N}$ \\
\hline TN J & $2938 \pm 125$ & $447.9 \pm 9.2$ & $\ldots$ & \pm 0.04 & $\ldots$ & $\ldots$ & $\ldots$ & $\ldots$ & 11333 & -271522.9 & $\mathrm{~N}$ \\
\hline TN J1136+1551 & $384 \pm 33$ & $21.7 \pm 0.6$ & ... & $-2.14 \pm 0.07$ & $\ldots$ & $\ldots$ & $\ldots$ & $\ldots$ & 113603.28 & +155106.7 & $\mathrm{~N}$ \\
\hline TN J1136 & $88 \pm 50$ & \pm 5.3 & $\ldots$ & $-1.32 \pm 0.06$ & $\ldots$ & $\ldots$ & $\ldots$ & $\ldots$ & 1136 & +061026.8 & $\mathrm{~N}$ \\
\hline & \pm 58 & \pm 3.9 & $\ldots$ & -1 . & $\ldots$ & & & $\ldots$ & 1139 & +093526.1 & $\mathrm{N}$ \\
\hline & \pm 79 & \pm 5.4 & $\ldots$ & -1.4 & $\ldots$ & $\mathrm{S}$ & 8.8 & 1 & 1146 & -105208.6 & A \\
\hline $\mathrm{TN} \mathrm{J}$ & $571 \pm 67$ & $.4 \pm 3.7$ & $\ldots$ & $-1.46 \pm 0.09$ & $\ldots$ & $\mathrm{S}$ & $<6.0$ & 5 & 11483 & -090148.8 & A \\
\hline TN J1149+1844 & $457 \pm 39$ & $77.6 \pm 1.6$ & $\ldots$ & $-1.32 \pm 0.07$ & ... & $\ldots$ & $\ldots$ & ... & 114936.78 & +184421.5 & $\mathrm{~N}$ \\
\hline TN J & $5 \pm 28$ & \pm 1.2 & $7.0 \pm 0.4$ & $-1.57 \pm 0.05$ & $-1.62 \pm 0.05$ & $\mathrm{~S}$ & 1.0 & 0 & 1151 & -301340.8 & V \\
\hline & \pm 48 & \pm 2.3 & & \pm 0.12 & & $\mathrm{~S}$ & 10.7 & 167 & 1156 & -31054 & A \\
\hline & $4 \pm 34$ & $52.9 \pm 1.2$ & $10.4 \pm 0.6$ & $-1.35 \pm 0.08$ & $-1.30 \pm 0.05$ & $\mathrm{D}$ & 1.4 & 38 & 11595 & -162948.2 & $\mathrm{~V}$ \\
\hline & $4 \pm 56$ & $129.7 \pm 5.8$ & $\ldots$ & $-1.36 \pm 0.06$ & $\ldots$ & $\ldots$ & $\ldots$ & $\ldots$ & 12042 & +163049.5 & $\mathrm{~N}$ \\
\hline TN J1210+1738 & $397 \pm 50$ & $68.1 \pm 3.2$ & $\ldots$ & $-1.31 \pm 0.10$ & ... & $\ldots$ & $\ldots$ & ... & 121036.00 & +173827.8 & $\mathrm{~N}$ \\
\hline+1944 & \pm 44 & \pm 4.1 & $\ldots$ & $33 \pm 0.07$ & $\ldots$ & & & $\cdots$ & 216 & +194 & $\mathrm{~N}$ \\
\hline & \pm 43 & \pm 6.4 & $\ldots$ & -1 . & $\ldots$ & $\mathrm{S}$ & $<7.0$ & 163 & 1216 & -28504 & A \\
\hline & \pm 61 & \pm 3.0 & $\ldots$ & -1 . & $\ldots$ & $\ldots$ & & $\ldots$ & 1220 & +060416.5 & $\mathrm{~N}$ \\
\hline & \pm 82 & \pm 9.5 & $\ldots$ & -1 & $\ldots$ & $\mathrm{S}$ & 8.3 & 162 & 221 & -26463 & A \\
\hline TN J1227-2255 & $1 \pm 27$ & \pm 2.2 & $\ldots$ & $-1.35 \pm 0.08$ & ... & $\mathrm{S}$ & $<7.0$ & 158 & 122747.23 & -225535.1 & A \\
\hline & $=89$ & $=1.7$ & $\ldots$ & +08 & $\cdots$ & .. & $\cdots$ & $\cdots$ & 39 & +100 & $\mathrm{~N}$ \\
\hline & \pm 63 & \pm 3.8 & $\cdots$ & -1 . & $\ldots$ & $\cdots$ & $\ldots$ & $\ldots$ & 1239 & +10432 & $\mathrm{~N}$ \\
\hline TN J & $29 \pm 74$ & \pm 12.2 & $\ldots$ & -1. & $\ldots$ & $\ldots$ & $\ldots$ & $\ldots$ & 1239 & -03190 & $\mathrm{~N}$ \\
\hline & $8 \pm 62$ & \pm 4.0 & $\ldots$ & -1 & $\ldots$ & $\ldots$ & $\ldots$ & $\ldots$ & 1245 & -11272 & $\mathrm{~N}$ \\
\hline TN J1247+1547 & $405 \pm 44$ & $70.1 \pm 3.2$ & ... & $-1.30 \pm 0.09$ & ... & $\ldots$ & $\ldots$ & $\ldots$ & 124704.84 & +154738.9 & $\mathrm{~N}$ \\
\hline & \pm 53 & \pm 4.3 & $\ldots$ & -1 . & $\ldots$ & $\mathrm{S}$ & 7.0 & 158 & 6 & -27141 & A \\
\hline & \pm 38 & \pm 3 & $\ldots$ & -1 . & $\ldots$ & $\ldots$ & $\ldots$ & $\ldots$ & 56 & -091124.8 & $\mathrm{~N}$ \\
\hline & \pm 108 & & $\ldots$ & & $\ldots$ & $\ldots$ & $\ldots$ & $\ldots$ & 7 & -33491 & $\mathrm{~N}$ \\
\hline & \pm 57 & & & & $\ldots$ & $\cdots$ & & $\ldots$ & 1306 & -043617.4 & $\mathrm{~N}$ \\
\hline TN J1313-0459 & $369 \pm 34$ & $58.1 \pm 2.7$ & ... & $-1.38 \pm 0.08$ & $\cdots$ & ... & $\ldots$ & ... & 131334.37 & -045930.0 & $\mathrm{~N}$ \\
\hline & \pm 62 & \pm 2.0 & $\cdots$ & -1 & $\cdots$ & & & $\cdots$ & 13172 & +03391 & $\mathrm{~N}$ \\
\hline & \pm 60 & +20 & $\ldots$ & -1 . & $\ldots$ & $\ldots$ & $\ldots$ & $\ldots$ & 13233 & $\begin{array}{llll}-26 & 04 & 04.7\end{array}$ & $\mathrm{~N}$ \\
\hline & \pm 33 & & $\ldots$ & & $\ldots$ & & $\ldots$ & $\ldots$ & 1326 & -233023.5 & $\mathrm{~N}$ \\
\hline & \pm 68 & & & & $\cdots$ & & & & 1327 & +14370 & $\mathrm{~N}$ \\
\hline TN J1336+2450 & $358 \pm 71$ & $51.3 \pm 2.5$ & $\cdots$ & $-1.45 \pm 0.15$ & $\ldots$ & M & 54.4 & 100 & 133615.12 & +245005.4 & $\mathrm{~F}$ \\
\hline & \pm 63 & \pm 5.4 & $.1 \pm 1.2$ & $-1.31 \pm 0$ & $-1.34 \pm 0.06$ & $\mathrm{~S}$ & 1.4 & 152 & 38 & -19423 & V \\
\hline & & & & \pm 0.11 & & & & & 13395 & $-0114 \quad 10.8$ & $\mathrm{~N}$ \\
\hline & $7 \pm 93$ & $137.8 \pm 6.1$ & $24.8 \pm 1.4$ & $-1.33 \pm 0.09$ & $-1.38 \pm 0.06$ & $\mathrm{DF}$ & 1.7 & 0 & 134029.38 & +272325.0 & V \\
\hline TN J & $3 \pm 59$ & $49.6 \pm 2.4$ & & $-1.48 \pm 0.13$ & $\ldots$ & & $\ldots$ & $\ldots$ & $1346 \quad 16.84$ & -100457.2 & $\mathrm{~N}$ \\
\hline TN J1351+1328 & $662 \pm 54$ & $93.8 \pm 4.2$ & & $-1.45 \pm 0.07$ & $\cdots$ & & $\ldots$ & $\ldots$ & 135154.72 & +132838.1 & $\mathrm{~N}$ \\
\hline
\end{tabular}


Table A.2. continued

\begin{tabular}{|c|c|c|c|c|c|c|c|c|c|c|c|c|}
\hline Name & $\begin{array}{l}S_{365} \\
\mathrm{mJy}\end{array}$ & $\begin{array}{r}S_{1400} \\
\mathrm{mJy}\end{array}$ & $\begin{array}{r}S_{4850} \\
\mathrm{mJy} \\
\end{array}$ & $\alpha_{365}^{1400}$ & $\alpha_{1400}^{4850}$ & Str & $\begin{array}{r}\text { LAS } \\
\prime \prime\end{array}$ & $\begin{array}{r}\mathrm{PA} \\
\circ\end{array}$ & $\mathrm{h} \quad{ }_{\mathrm{m}}^{\alpha_{\mathrm{J} 2000}} \mathrm{~s}_{\mathrm{s}}$ & $\circ$ & $\delta_{\mathrm{J}} 2000$ & Pos \\
\hline TN J1352-0800 & $554 \pm 88$ & $95.6 \pm 4.3$ & & $-1.31 \pm 0.12$ & & & $\ldots$ & $\ldots$ & 135255.32 & -08 & $\overline{0014.1}$ & $\mathrm{~N}$ \\
\hline TN J1353+2302 & $403 \pm 38$ & $59.5 \pm 1.3$ & $23.3 \pm 1.3$ & $-1.42 \pm 0.07$ & $-0.75 \pm 0.05$ & DF & 2.5 & 44 & 135342.26 & +23 & 0250.9 & V \\
\hline TN J1358+2003 & $227 \pm 42$ & $35.0 \pm 1.8$ & $\ldots$ & $-1.39 \pm 0.14$ & $\ldots$ & $\ldots$ & $\ldots$ & $\ldots$ & 135829.63 & +20 & 0313.4 & $\mathrm{~N}$ \\
\hline TN J1403-1223 & $396 \pm 69$ & $65.8 \pm 3.0$ & $\ldots$ & $-1.34 \pm 0.14$ & $\ldots$ & $\mathrm{S}$ & 30.6 & 169 & 140337.33 & -12 & 2357.3 & A \\
\hline TN J1408-0855 & $694 \pm 73$ & $114.3 \pm 5.1$ & $\ldots$ & $-1.34 \pm 0.09$ & $\ldots$ & $\cdots$ & $\ldots$ & $\ldots$ & 140818.46 & -08 & 5538.5 & $\mathrm{~N}$ \\
\hline TN J1411-0824 & $542 \pm 71$ & $76.1 \pm 3.5$ & $\ldots$ & $-1.46 \pm 0.10$ & $\ldots$ & $\ldots$ & $\ldots$ & $\ldots$ & 141153.33 & -08 & 2402.9 & $\mathrm{~N}$ \\
\hline TN J1412-2548 & $452 \pm 59$ & $78.0 \pm 3.6$ & $\ldots$ & $-1.31 \pm 0.10$ & $\ldots$ & $\ldots$ & $\ldots$ & $\ldots$ & 141251.27 & -25 & $48 \quad 05.5$ & $\mathrm{~N}$ \\
\hline TN J1418-2256 & $527 \pm 59$ & $82.0 \pm 3.7$ & $\ldots$ & $-1.38 \pm 0.09$ & $\ldots$ & $\mathrm{S}$ & 7.6 & 148 & $1418 \quad 16.65$ & -22 & 5658.6 & A \\
\hline TN J1428+2425 & $411 \pm 34$ & $69.1 \pm 1.5$ & $\ldots$ & $-1.33 \pm 0.07$ & $\ldots$ & $\mathrm{S}$ & 1.0 & 0 & $1428 \quad 10.20$ & +24 & 2511.3 & $\mathrm{~F}$ \\
\hline TN J1438+2334 & $564 \pm 69$ & $96.5 \pm 4.2$ & $\cdots$ & $-1.31 \pm 0.10$ & $\cdots$ & $\mathrm{D}$ & 21.1 & 157 & 143856.52 & +23 & 3425.6 & $\mathrm{~F}$ \\
\hline TN J1439-3226 & $622 \pm 65$ & $90.7 \pm 1.9$ & $\ldots$ & $-1.43 \pm 0.08$ & $\ldots$ & $\ldots$ & $\ldots$ & $\ldots$ & 143902.87 & -32 & 2646.6 & $\mathrm{~N}$ \\
\hline TN J1449+1440 & $464 \pm 50$ & $80.3 \pm 3.7$ & $\ldots$ & $-1.30 \pm 0.09$ & $\ldots$ & $\ldots$ & $\ldots$ & $\ldots$ & 144902.50 & +14 & 4042.2 & $\mathrm{~N}$ \\
\hline TN J1451+2351 & $595 \pm 44$ & $93.2 \pm 4.2$ & $\ldots$ & $-1.38 \pm 0.07$ & $\ldots$ & D & 12.6 & 109 & $1451 \quad 10.71$ & +23 & 5138.8 & $\mathrm{~F}$ \\
\hline TN J1452-1113 & $344 \pm 55$ & $53.9 \pm 2.6$ & $\ldots$ & $-1.38 \pm 0.13$ & $\ldots$ & $\mathrm{S}$ & $<7.5$ & 2 & $1452 \quad 04.88$ & -11 & 1335.7 & A \\
\hline TN J1452+2013 & $291 \pm 40$ & $43.4 \pm 1.0$ & $\ldots$ & $-1.42 \pm 0.11$ & $\cdots$ & $\cdots$ & $\ldots$ & $\cdots$ & 145222.93 & +20 & 1309.8 & $\mathrm{~N}$ \\
\hline TN J1453+1106 & $833 \pm 56$ & $144.9 \pm 6.4$ & $\ldots$ & $-1.30 \pm 0.06$ & $\ldots$ & $\ldots$ & $\ldots$ & $\ldots$ & 145321.97 & +11 & $06 \quad 04.9$ & $\mathrm{~N}$ \\
\hline TN J1454+0017 & $1309 \pm 88$ & $214.8 \pm 9.2$ & $\ldots$ & $-1.34 \pm 0.06$ & $\ldots$ & $\ldots$ & $\ldots$ & $\ldots$ & 145440.87 & +00 & 1741.9 & $\mathrm{~N}$ \\
\hline TN J1459-2730 & $321 \pm 40$ & $52.5 \pm 2.5$ & $\ldots$ & $-1.35 \pm 0.10$ & $\ldots$ & $\mathrm{S}$ & 6.5 & 143 & 145934.96 & -27 & 3008.3 & A \\
\hline TN J1506+2728 & $360 \pm 58$ & $60.7 \pm 1.3$ & $15.9 \pm 0.9$ & $-1.32 \pm 0.12$ & $-1.07 \pm 0.05$ & D & 1.1 & 131 & 150635.41 & +27 & 2855.6 & $\mathrm{~V}$ \\
\hline TN J1513-2417 & $504 \pm 33$ & $83.6 \pm 3.7$ & $\ldots$ & $-1.34 \pm 0.06$ & $\cdots$ & $\cdots$ & $\cdots$ & $\ldots$ & 151331.67 & -24 & 1755.3 & $\mathrm{~N}$ \\
\hline TN J1513-1801 & $534 \pm 36$ & $85.3 \pm 3.9$ & $\ldots$ & $-1.36 \pm 0.06$ & $\ldots$ & $\mathrm{S}$ & $<7.5$ & 4 & 151355.31 & -18 & $\begin{array}{lll}0 & 07.9\end{array}$ & A \\
\hline TN J1515-2651 & $352 \pm 44$ & $58.0 \pm 2.7$ & $\ldots$ & $-1.34 \pm 0.10$ & $\ldots$ & $\mathrm{S}$ & 6.5 & 9 & $15 \quad 15 \quad 59.29$ & -26 & 5114.9 & A \\
\hline TN J1520+1410 & $478 \pm 55$ & $66.0 \pm 1.4$ & $\ldots$ & $-1.47 \pm 0.09$ & $\ldots$ & $\ldots$ & $\ldots$ & $\ldots$ & 152053.36 & +14 & 1045.5 & $\mathrm{~N}$ \\
\hline TN J1521+0741 & $1777 \pm 123$ & $105.6 \pm 4.5$ & $\ldots$ & $-2.10 \pm 0.06$ & $\ldots$ & $\ldots$ & $\ldots$ & $\ldots$ & 152150.75 & +07 & 4141.1 & $\mathrm{~N}$ \\
\hline TN J1522-2540 & $464 \pm 63$ & $54.1 \pm 2.6$ & $\cdots$ & $-1.60 \pm 0.11$ & $\cdots$ & $\mathrm{S}$ & 8.2 & 10 & 152223.28 & -25 & $\begin{array}{ll}40 & 07.1\end{array}$ & A \\
\hline TN J1524+0642 & $479 \pm 55$ & $82.9 \pm 1.7$ & $\ldots$ & $-1.30 \pm 0.09$ & $\cdots$ & $\cdots$ & $\ldots$ & $\ldots$ & 152406.39 & +06 & $42 \quad 15.9$ & $\mathrm{~N}$ \\
\hline TN J1531-3234 & $522 \pm 59$ & $80.6 \pm 3.6$ & $\ldots$ & $-1.39 \pm 0.09$ & $\ldots$ & $\mathrm{S}$ & 4.8 & 27 & 153133.59 & -32 & 3401.7 & A \\
\hline TN J1547-2218 & $427 \pm 62$ & $72.4 \pm 3.3$ & $\ldots$ & $-1.32 \pm 0.11$ & $\ldots$ & $\ldots$ & $\ldots$ & $\ldots$ & 154749.95 & -22 & 1825.6 & $\mathrm{~N}$ \\
\hline TN J1556-2759 & $612 \pm 56$ & $66.4 \pm 3.1$ & $\ldots$ & $-1.65 \pm 0.08$ & $\ldots$ & $\ldots$ & $\ldots$ & $\ldots$ & 155643.63 & -27 & 5943.1 & $\mathrm{~N}$ \\
\hline TN J1559-1727 & $575 \pm 71$ & $97.4 \pm 4.3$ & $\cdots$ & $-1.32 \pm 0.10$ & $\cdots$ & $\cdots$ & $\cdots$ & $\cdots$ & 155934.08 & -17 & 2741.6 & $\mathrm{~N}$ \\
\hline TN J1604-0248 & $345 \pm 33$ & $46.8 \pm 1.0$ & $\ldots$ & $-1.49 \pm 0.07$ & $\ldots$ & $\ldots$ & $\ldots$ & $\ldots$ & $16 \quad 0459.59$ & -02 & $48 \quad 47.9$ & $\mathrm{~N}$ \\
\hline TN J1621+2043 & $539 \pm 48$ & $61.4 \pm 2.9$ & $\ldots$ & $-1.62 \pm 0.08$ & $\ldots$ & $\ldots$ & $\ldots$ & $\ldots$ & 162106.18 & +20 & 4336.4 & $\mathrm{~N}$ \\
\hline TN J1628+1604 & $602 \pm 44$ & $55.5 \pm 2.6$ & $\ldots$ & $-1.77 \pm 0.07$ & $\ldots$ & $\ldots$ & $\ldots$ & $\ldots$ & $16 \quad 28 \quad 19.16$ & +16 & 0400.0 & $\mathrm{~N}$ \\
\hline TN J1634-2222 & $4714 \pm 214$ & $809.2 \pm 35.4$ & $\ldots$ & $-1.31 \pm 0.05$ & $\ldots$ & $\ldots$ & $\ldots$ & $\ldots$ & $16 \quad 3449.79$ & -22 & 2212.7 & $\mathrm{~N}$ \\
\hline TN J1637-1931 & $432 \pm 40$ & $36.8 \pm 0.9$ & $\cdots$ & $-1.83 \pm 0.07$ & $\cdots$ & $\mathrm{S}$ & 10.8 & 6 & 163744.80 & -19 & 3124.8 & A \\
\hline TN J1646-1328 & $608 \pm 63$ & $102.5 \pm 4.6$ & $\ldots$ & $-1.32 \pm 0.09$ & $\cdots$ & $\cdots$ & $\cdots$ & $\ldots$ & 164651.67 & -13 & 2849.1 & $\mathrm{~N}$ \\
\hline TN J1652-1339 & $328 \pm 33$ & $56.5 \pm 1.3$ & $\ldots$ & $-1.31 \pm 0.08$ & $\ldots$ & $\ldots$ & $\ldots$ & $\ldots$ & $\begin{array}{lll}16 & 52 & 24.29\end{array}$ & -13 & 3904.3 & $\mathrm{~N}$ \\
\hline TN J1653-1155 & $1131 \pm 60$ & $197.0 \pm 8.7$ & $\ldots$ & $-1.30 \pm 0.05$ & $\ldots$ & $\ldots$ & $\ldots$ & $\ldots$ & 165352.81 & -11 & 5559.2 & $\mathrm{~N}$ \\
\hline TN J1656-1521 & $587 \pm 79$ & $93.9 \pm 4.2$ & $\ldots$ & $-1.36 \pm 0.11$ & $\ldots$ & $\ldots$ & $\ldots$ & $\ldots$ & $\begin{array}{lll}16 & 56 & 03.77\end{array}$ & -15 & 2123.3 & $\mathrm{~N}$ \\
\hline TN J1701-0101 & $999 \pm 55$ & $172.2 \pm 7.6$ & $\cdots$ & $-1.31 \pm 0.05$ & $\cdots$ & $\cdots$ & $\cdots$ & $\cdots$ & 170137.58 & -01 & 0113.6 & $\mathrm{~N}$ \\
\hline TN J1701+0252 & $356 \pm 38$ & $57.1 \pm 2.7$ & $\ldots$ & $-1.36 \pm 0.09$ & $\cdots$ & $\cdots$ & $\ldots$ & $\ldots$ & 170154.89 & +02 & 5213.4 & $\mathrm{~N}$ \\
\hline TN J1702-0811 & $436 \pm 38$ & $69.9 \pm 3.2$ & $\ldots$ & $-1.36 \pm 0.07$ & $\ldots$ & $\ldots$ & $\ldots$ & $\ldots$ & $\begin{array}{lll}17 & 02 & 04.71\end{array}$ & -08 & 1107.5 & $\mathrm{~N}$ \\
\hline TN J1702+2015 & $322 \pm 48$ & $43.4 \pm 2.1$ & $\ldots$ & $-1.49 \pm 0.12$ & $\ldots$ & $\ldots$ & $\ldots$ & $\ldots$ & $\begin{array}{lll}17 & 02 & 23.02\end{array}$ & +20 & 1534.2 & $\mathrm{~N}$ \\
\hline TN J1702-0145 & $808 \pm 74$ & $135.6 \pm 6.0$ & $\ldots$ & $-1.33 \pm 0.08$ & $\ldots$ & $\ldots$ & $\ldots$ & $\ldots$ & $\begin{array}{lll}17 & 02 & 43.11\end{array}$ & -01 & 4554.0 & $\mathrm{~N}$ \\
\hline TN J1707-1120 & $425 \pm 72$ & $54.2 \pm 2.6$ & $\cdots$ & $-1.53 \pm 0.13$ & $\cdots$ & $\cdots$ & $\cdots$ & $\ldots$ & 170751.37 & -11 & 2021.0 & $\mathrm{~N}$ \\
\hline TN J1710-0826 & $445 \pm 74$ & $69.2 \pm 3.2$ & $\ldots$ & $-1.38 \pm 0.13$ & $\cdots$ & $\cdots$ & $\ldots$ & $\ldots$ & 171020.36 & -08 & 2640.3 & $\mathrm{~N}$ \\
\hline TN J1714+2226 & $285 \pm 48$ & $43.3 \pm 1.0$ & $\ldots$ & $-1.40 \pm 0.13$ & $\ldots$ & $\mathrm{S}$ & 1.6 & 38 & 171453.42 & +22 & 2618.5 & $\mathrm{~F}$ \\
\hline TN J1821+2433 & $1416 \pm 95$ & $227.5 \pm 4.7$ & $\ldots$ & $-1.36 \pm 0.05$ & $\ldots$ & $\ldots$ & $\ldots$ & $\ldots$ & 182137.07 & +24 & 3313.8 & $\mathrm{~N}$ \\
\hline TN J1855-3430 & $654 \pm 85$ & $87.8 \pm 3.8$ & $\ldots$ & $-1.49 \pm 0.10$ & $\ldots$ & $\ldots$ & $\ldots$ & $\ldots$ & 185524.12 & -34 & 3027.7 & $\mathrm{~N}$ \\
\hline TN J1905-3520 & $799 \pm 75$ & $131.3 \pm 5.4$ & $\cdots$ & $-1.34 \pm 0.08$ & $\cdots$ & $\cdots$ & $\cdots$ & $\ldots$ & 190522.67 & -35 & 2050.9 & $\mathrm{~N}$ \\
\hline TN J1932-1931 & $5678 \pm 335$ & $813.6 \pm 35.5$ & $\ldots$ & $-1.45 \pm 0.05$ & $\cdots$ & $\cdots$ & $\ldots$ & $\ldots$ & $19 \quad 32 \quad 07.22$ & -19 & 3149.7 & $\mathrm{~N}$ \\
\hline TN J1941-1952 & $1194 \pm 103$ & $183.0 \pm 3.8$ & $\ldots$ & $-1.40 \pm 0.07$ & $\ldots$ & $\mathrm{S}$ & 7.9 & 164 & 194100.07 & -19 & 5214.0 & A \\
\hline TN J1953-0541 & $478 \pm 83$ & $59.0 \pm 2.8$ & $\ldots$ & $-1.56 \pm 0.13$ & $\ldots$ & $\ldots$ & $\ldots$ & $\ldots$ & 195327.04 & -05 & 4128.5 & $\mathrm{~N}$ \\
\hline TN J1954-1207 & $657 \pm 67$ & $102.8 \pm 4.6$ & $\ldots$ & $-1.38 \pm 0.08$ & $\ldots$ & $\mathrm{S}$ & $<7.0$ & 177 & 195424.15 & -12 & 0748.7 & A \\
\hline TN J2007-1316 & $777 \pm 46$ & $115.4 \pm 5.2$ & $\cdots$ & $-1.42 \pm 0.06$ & $\cdots$ & $\mathrm{S}$ & 7.2 & 174 & 200753.23 & -13 & 1643.6 & A \\
\hline TN J2008-1344 & $798 \pm 71$ & $132.3 \pm 2.7$ & $\cdots$ & $-1.34 \pm 0.07$ & $\ldots$ & $\mathrm{S}$ & 8.2 & 176 & $\begin{array}{llll}20 & 08 & 07.48\end{array}$ & -13 & 4417.8 & A \\
\hline TN J2009-3040 & $409 \pm 65$ & $65.3 \pm 3.0$ & $\ldots$ & $-1.36 \pm 0.12$ & $\ldots$ & $\mathrm{S}$ & $<7.0$ & 144 & 200948.13 & -30 & 4007.0 & A \\
\hline TN J2014-2115 & $348 \pm 33$ & $48.0 \pm 1.1$ & $\cdots$ & $-1.47 \pm 0.07$ & $\cdots$ & $\mathrm{D}$ & 56.4 & 11 & 201431.96 & -21 & 1436.7 & A \\
\hline TN J2021+0839 & $880 \pm 72$ & $145.4 \pm 6.4$ & ... & $-1.34 \pm 0.07$ & $\ldots$ & $\ldots$ & $\ldots$ & $\ldots$ & 202133.11 & +08 & 3909.2 & $\mathrm{~N}$ \\
\hline TN J2028+0811 & $455 \pm 58$ & $74.8 \pm 1.6$ & $\ldots$ & $-1.34 \pm 0.10$ & $\ldots$ & $\ldots$ & $\ldots$ & $\ldots$ & 202837.12 & +08 & 1121.4 & $\mathrm{~N}$ \\
\hline
\end{tabular}


Table A.2. continued

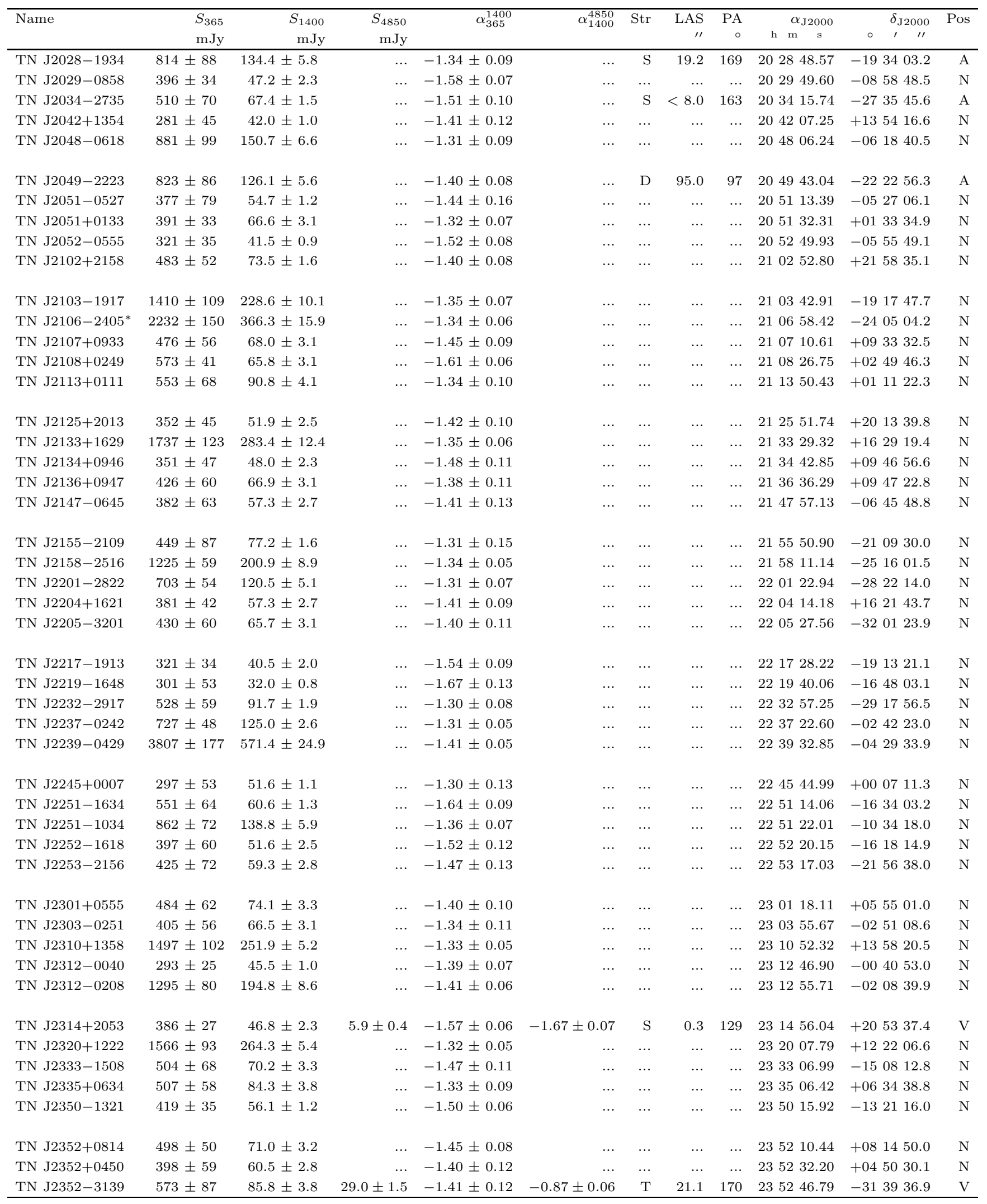

$\dagger$ Not a real USS source; see notes.

* See notes. 
Table A.3. MP sample

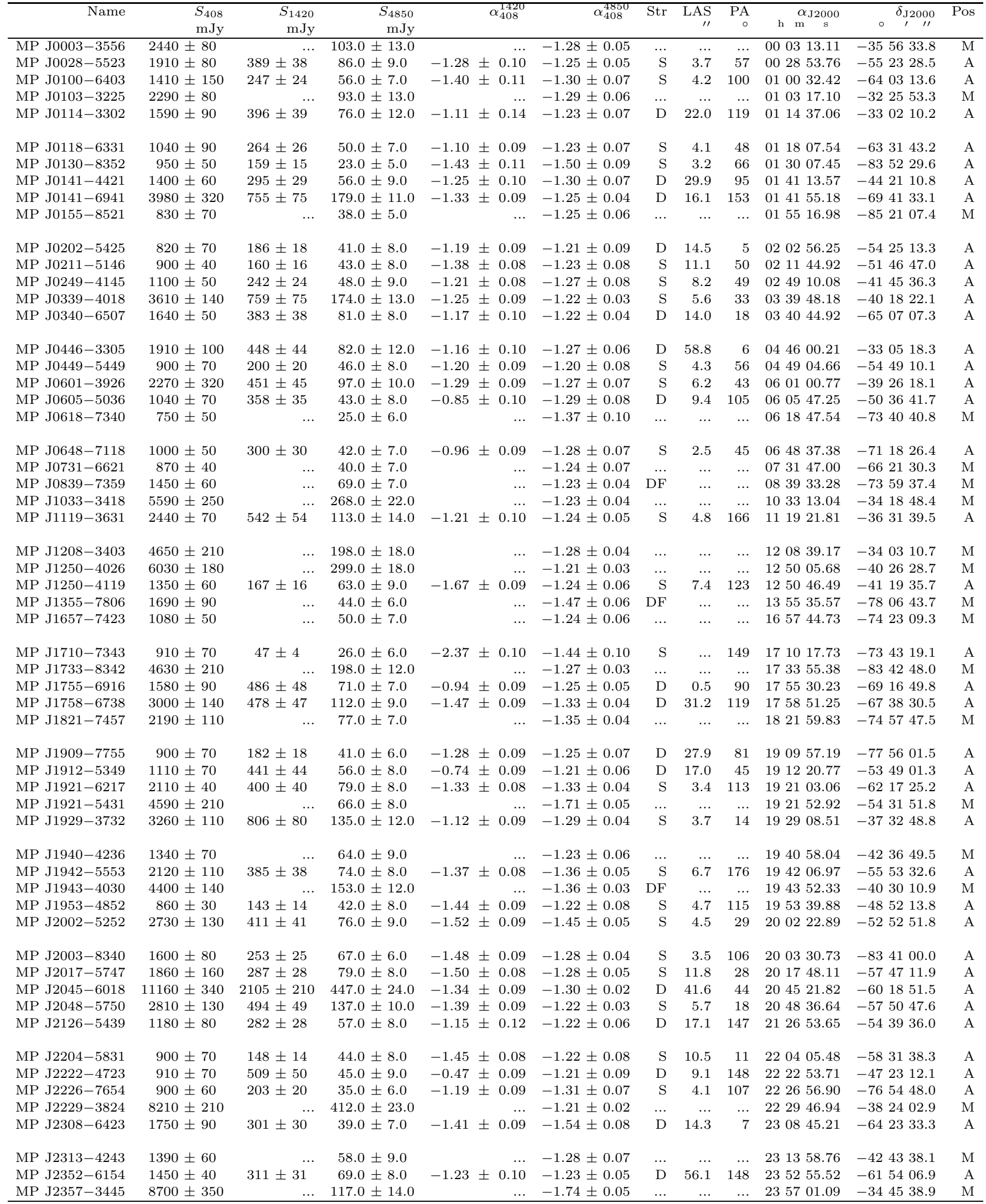


Table A.4. WN POSS identifications

\begin{tabular}{|c|c|c|c|c|}
\hline Name & $\begin{array}{c}S_{1400} \\
\mathrm{mJy}\end{array}$ & $\alpha_{325}^{1400}$ & $\begin{array}{r}\mathrm{LAS} \\
\prime \prime\end{array}$ & $\mathrm{LR}^{a}$ \\
\hline WN J0029+3439 & 40.9 & -1.34 & 12.8 & 4.2 \\
\hline WN J0121+4305 & 50.8 & -1.39 & $\ldots$ & 7.1 \\
\hline WN J0315+3757 & 13.3 & -1.35 & 1.7 & 14.8 \\
\hline WN J0559+6926 & 12.9 & -1.31 & 0.3 & 4.6 \\
\hline WN J0641+4325 & 15.6 & -1.57 & $\ldots$ & 195.6 \\
\hline WN J0725+4123 & 35.5 & -1.39 & 8.3 & 183.0 \\
\hline WN J0756+5010 & 29.9 & -1.31 & 1.7 & 127.3 \\
\hline WN J0830+3018 & 13.9 & -1.41 & 3.5 & 27.3 \\
\hline WN J0835+3439 & 19.1 & -1.38 & 58.0 & 24.6 \\
\hline WN J0923+4602 & 12.1 & -1.49 & 21.6 & 17.0 \\
\hline WN J0952+5153 & 16.1 & -1.76 & 2.7 & 194.1 \\
\hline WN J0955+6023 & 10.9 & -1.54 & 3.1 & 1.0 \\
\hline WN J1014+7407 & 21.8 & -1.41 & 2.2 & 147.7 \\
\hline WN J1026+2943 & 12.0 & -1.31 & 11.9 & 136.5 \\
\hline WN J1030+5415 & 29.2 & -1.39 & 6.1 & 16.4 \\
\hline WN J1052+4826 & 154.2 & -1.31 & 10.0 & 38.1 \\
\hline WN J1124+3228 & 38.9 & -1.31 & 5.7 & 101.2 \\
\hline WN J1130+4911 & 18.9 & -1.34 & 37.0 & 18.9 \\
\hline WN J1141+6924 & 11.8 & -1.32 & $\ldots$ & 1.4 \\
\hline WN J1148+5116 & 20.5 & -1.35 & 6.7 & 1.0 \\
\hline WN J1152+3732 & 17.1 & -2.18 & 15.0 & 159.9 \\
\hline WN J1232+4621 & 19.8 & -1.30 & 12.5 & 42.9 \\
\hline WN J1258+5041 & 28.8 & -1.42 & 44.9 & 3.7 \\
\hline WN J1259+3121 & 34.0 & -1.41 & 20.6 & 0.0 \\
\hline WN J1330+6505 & 10.5 & -1.34 & 1.8 & 21.5 \\
\hline WN J1353+3336 & 15.9 & -1.31 & $\ldots$ & 3.3 \\
\hline WN J1400+4348 & 20.2 & -1.49 & 6.4 & 29.2 \\
\hline WN J1403+3109 & 45.7 & -1.35 & 11.0 & 148.5 \\
\hline WN J1410+4615 & 14.2 & -1.36 & 4.4 & 42.5 \\
\hline WN J1440+3707 & 16.7 & -1.76 & 7.4 & 182.0 \\
\hline WN J1459+4947 & 18.7 & -1.53 & 3.0 & 47.2 \\
\hline WN J1558+7028 & 15.7 & -1.36 & 2.1 & 2.8 \\
\hline WN J1624+4202 & 11.0 & -1.31 & 24.4 & 182.2 \\
\hline WN J1628+3932 & 3680.7 & -1.36 & 48.8 & 0.0 \\
\hline WN J1717+3828 & 35.8 & -1.60 & 33.6 & 141.4 \\
\hline WN J1752+2949 & 16.9 & -1.42 & 3.2 & 3.4 \\
\hline WN J1801+3336 & 11.6 & -1.69 & 2.5 & 1.5 \\
\hline WN J1815+3656 & 17.6 & -1.34 & 1.6 & 195.0 \\
\hline WN J1819+6213 & 12.7 & -1.45 & 1.6 & 122.9 \\
\hline WN J1852+5711 & 53.4 & -1.36 & $\ldots$ & 145.4 \\
\hline WN J1927+6436 & 80.3 & -1.44 & 20.1 & 6.2 \\
\hline WN J1944+6552 & 147.2 & -1.53 & 1.8 & 147.5 \\
\hline WN J2146+3330 & 10.8 & -1.56 & $\ldots$ & 4.6 \\
\hline WN J2147+3137 & 25.4 & -1.46 & $\ldots$ & 31.9 \\
\hline WN J2313+3842 & 11.3 & -1.50 & $\ldots$ & 0.0 \\
\hline WN J2313+4053 & 40.2 & -1.42 & 1.5 & 36.4 \\
\hline
\end{tabular}

${ }^{a}$ Likelihood Ratio, see text.
Table A.5. TN POSS identifications

\begin{tabular}{lrrrr}
\hline Name & $S_{1400}$ & $\alpha_{365}^{1400}$ & $\begin{array}{r}\text { LAS } \\
\text { mJy }\end{array}$ & LR $^{a}$ \\
\hline TN J0244+0327 & 84.8 & -1.38 & $\ldots$ & 6.9 \\
TN J0245+2700 & 54.0 & -1.47 & $\ldots$ & 30.5 \\
TN J0250+0130 & 39.1 & -1.45 & $\ldots$ & 14.7 \\
TN J0256-2717 & 177.9 & -1.32 & $\ldots$ & 126.6 \\
TN J0301+0155 & 402.7 & -1.70 & $\ldots$ & 21.3 \\
TN J0433+0717 & 33.1 & -1.44 & $\ldots$ & 29.9 \\
TN J0443-1212 & 56.8 & -1.47 & 20.2 & 162.1 \\
TN J0510-1838 & 641.3 & -1.76 & 36.1 & 0.0 \\
TN J0729+2436 & 68.9 & -2.03 & 19.4 & 129.0 \\
TN J0812+0915 & 43.4 & -1.38 & $\ldots$ & 37.7 \\
TN J0818-0741 & 59.3 & -1.38 & $\ldots$ & 96.5 \\
TN J0958-1103 & 70.6 & -1.35 & $\ldots$ & 181.8 \\
TN J1043+2404 & 52.0 & -1.59 & 3.7 & 167.8 \\
TN J1053-1518 & 63.7 & -1.33 & $\ldots$ & 1.8 \\
TN J1117-1409 & 134.5 & -1.31 & $\ldots$ & 128.0 \\
TN J1220+0604 & 65.1 & -1.50 & $\ldots$ & 189.4 \\
TN J1239+1005 & 79.3 & -1.75 & $\ldots$ & 180.7 \\
TN J1245-1127 & 88.0 & -1.32 & $\ldots$ & 146.1 \\
TN J1326-2330 & 83.2 & -1.37 & $\ldots$ & 190.1 \\
TN J1408-0855 & 114.3 & -1.34 & $\ldots$ & 160.9 \\
TN J1418-2256 & 82.0 & -1.38 & 7.6 & 85.4 \\
TN J1513-2417 & 83.6 & -1.34 & $\ldots$ & 2.5 \\
TN J1521+0741 & 105.6 & -2.10 & $\ldots$ & 0.0 \\
TN J1531-3234 & 80.6 & -1.39 & 4.8 & 172.5 \\
TN J1547-2218 & 72.4 & -1.32 & $\ldots$ & 7.1 \\
TN J1556-2759 & 66.4 & -1.65 & $\ldots$ & 9.2 \\
TN J1628+1604 & 55.5 & -1.77 & $\ldots$ & 153.7 \\
TN J1646-1328 & 102.5 & -1.32 & $\ldots$ & 119.7 \\
TN J1701+0252 & 57.1 & -1.36 & $\ldots$ & 19.4 \\
TN J1707-1120 & 54.2 & -1.53 & $\ldots$ & 85.5 \\
TN J1710-0826 & 69.2 & -1.38 & $\ldots$ & 8.8 \\
TN J1953-0541 & 59.0 & -1.56 & $\ldots$ & 71.3 \\
TN J2028+0811 & 74.8 & -1.34 & $\ldots$ & 33.1 \\
TN J2155-2109 & 77.2 & -1.31 & $\ldots$ & 184.6 \\
TN J2335+0634 & 84.3 & -1.33 & $\ldots$ & 189.2 \\
\hline & & & &
\end{tabular}

${ }^{a}$ Likelihood Ratio, see text.

Table A.6. MP UKST identifications

\begin{tabular}{rrrrr}
\hline Name & $\begin{array}{r}S_{408} \\
\text { mJy }\end{array}$ & $\alpha_{408}^{4850}$ & $\begin{array}{r}\text { LAS } \\
\prime \prime\end{array}$ & LR $^{a}$ \\
\hline MP J0003-3556 & 2440 & -1.28 & $\ldots$ & 0.0 \\
MP J0103-3225 & 2290 & -1.29 & $\ldots$ & 88.1 \\
MP J0618-7340 & 750 & -1.37 & $\ldots$ & 7.5 \\
MP J1033-3418 & 5590 & -1.23 & $\ldots$ & 81.7 \\
MP J1250-4026 & 1350 & -1.24 & 7.4 & 131.0 \\
MP J1921-5431 & 4590 & -1.71 & $\ldots$ & 0.0 \\
MP J1943-4030 & 4400 & -1.36 & $\ldots$ & 0.0 \\
MP J2313-4243 & 1390 & -1.28 & $\ldots$ & 95.5 \\
MP J2357-3445 & 8700 & -1.74 & $\ldots$ & 0.0 \\
\hline
\end{tabular}

${ }^{a}$ Likelihood Ratio, see text. 
Table A.7. WN identifications from the literature

\begin{tabular}{rrrrrr}
\hline Name & $z$ & $R[\mathrm{mag}]$ & $F_{\mathrm{X}}^{a}$ & Identification & Reference \\
\hline WN J0633+4653 & $\ldots$ & 23.6 & $\ldots$ & $4 \mathrm{C}+46.12$ & Chambers et al. 1996a \\
WN J0648+4309 & $\ldots$ & 23.0 & $\ldots$ & B3 0644+432 & Wieringa \& Katgert 1992 \\
WN J0658+4444 & $\ldots$ & 23.4 & $\ldots$ & B3 0654+448 & Wieringa \& Katgert 1992 \\
WN J0717+4611 & 1.462 & 21.6 & $\ldots$ & B3 0714+462 & De Breuck et al. 1998b \\
WN J0725+4123 & $\ldots$ & $\ldots$ & 0.1137 & 1RXS J072600.0+4 & Voges et al. 1999 \\
WN J0923+4602 & $\ldots$ & $\ldots$ & 0.0242 & 1WGA J0923.1+460 & White et al. 1994 \\
WN J0952+5153 & 0.214 & $\ldots$ & 0.2413 & ZwCl 0949.6+5207 & Voges et al. 1999 \\
WN J1148+5116 & $\ldots$ & $\ldots$ & 0.0508 & 1RXS J114802.7+5 & Voges et al. 1999 \\
WN J1152+3732 & $\ldots$ & $\ldots$ & 0.0192 & 1WGA J1152.5+373 & White et al. 1994 \\
WN J1259+3121 & $\ldots$ & 16.2 & $\ldots$ & NGP9 F323-0140639 & Odewahn \& Aldering 1996 \\
WN J1332+3009 & $\ldots$ & 19.8 & $\ldots$ & NGP9 F324-0235590 & Odewahn \& Aldering 1996 \\
WN J1352+4259 & $\ldots$ & 21.7 & $\ldots$ & $4 C+43.31$ & Vigotti et al. 1989 \\
WN J1359+7447 & $\ldots$ & $\ldots$ & 0.07485 & 1RXS J135916.0+7 & Voges et al. 1999 \\
WN J1400+4348 & $\ldots$ & 8.2 & & HD122441 & SIMBAD \\
WN J1410+4615 & $\ldots$ & $\ldots$ & & SHK 010 & Shakhbazian 1973 \\
WN J1436+6319 & 4.261 & 24.1 & $\ldots$ & LC et al. 1994 \\
WN J1440+3707 & $\ldots$ & $\ldots$ & 0.07944 & 1RXS J144005.4+3 & Voges et al. 1999 \\
WN J1628+3932 & 0.031 & 13.1 & 4.5 & NGC 6166 & Zabludoff et al. 1993 \\
WN J1736+6502 & $\ldots$ & 22.2 & $\ldots$ & 8C 1736+650 & Lacy et al. 1993 \\
WN J1829+6913 & $\ldots$ & $\ldots$ & 0.05765 & 1RXS J182903.8+6 & Voges et al. 1999 \\
WN J1852+5711 & $\ldots$ & $\ldots$ & 0.1466 & 1RXS J185209.4+5 & Voges et al. 1999 \\
WN J1944+6552 & $\ldots$ & $\ldots$ & 0.1118 & 1RXS J194423.1+6 & Voges et al. 1999 \\
WN J2319+4251 & $\ldots$ & $\ldots$ & 0.1255 & 1RXS J231947.4+4 & Voges et al. 1999 \\
\hline
\end{tabular}

${ }^{a} F_{\mathrm{X}}$ is the number of X-ray counts $\mathrm{s}^{-1}$ as listed in the cited catalogs.

Table A.8. TN identifications from the literature

\begin{tabular}{|c|c|c|c|c|c|}
\hline Name & $z$ & $R[\mathrm{mag}]$ & $\overline{F_{\mathrm{X}}^{a}}$ & Identification & Reference \\
\hline TN J0055+2624 & 0.1971 & $\ldots$ & $\ldots$ & ABELL 85 & Giovannini et al. 1999 \\
\hline TN J0102-2152 & 0.0604 & $\ldots$ & $\ldots$ & ABELL 133 & Komissarov \& Gubanov 1994 \\
\hline TN J0245+2700 & & $\ldots$ & 0.0782 & 1RXS J024521.0+2 & Bade et al. 1998 \\
\hline TN J0256-2717 & 0.480 & 19.2 & $\ldots$ & MRC 0254-27 & McCarthy et al. 1996 \\
\hline TN J0301+0155 & 0.170 & $\ldots$ & 0.2053 & $\mathrm{ZwCl} 0258.9+0142$ & Crawford et al. 1995 \\
\hline TN J0408-2418 & 2.440 & 22.7 & $\ldots$ & MRC 0406-244 & McCarthy et al. 1996 \\
\hline TN J0510-1838 & $\ldots$ & 19.5 & 0.00314 & 1RXS J051032.4-1 & Voges et al. 1999 \\
\hline TN J0630-2834 & $\ldots$ & $\ldots$ & 0.00314 & 1WGA J0630.8-283 & White et al. 1994 \\
\hline TN J0729+2436 & $\ldots$ & $\ldots$ & 0.1752 & 1RXS J072927.4+2 & Voges et al. 1999 \\
\hline TN J0936-2243 & 1.339 & 23.0 & $\ldots$ & 3C 222 & Heckman et al. 1994 \\
\hline TN J0958-1103 & 0.153 & $\ldots$ & 1.64 & ABELL 0907 & McCarthy et al. 1996 \\
\hline TN J1521+0742 & 0.045 & 14.5 & 1.64 & NGC 5920 & Komissarov \& Gubanov 1994 \\
\hline TN J2106-2405 & 2.491 & 22.7 & $\ldots$ & MRC 2104-242 & McCarthy et al. 1996 \\
\hline TN J2239-0429 & $\ldots$ & 18.8 & $\ldots$ & $4 \mathrm{C}-04.85$ & Röttgering et al. 1995 \\
\hline TN J2320+1222 & $\ldots$ & 22.9 & $\ldots$ & MRC $2317+120$ & Röttgering et al. 1995 \\
\hline
\end{tabular}

${ }^{a} F_{\mathrm{X}}$ is the number of X-ray counts $\mathrm{s}^{-1}$ as listed in the cited catalogs.

Table A.9. MP identifications from the literature

\begin{tabular}{rrrrrr}
\hline Name & $z$ & $R[\mathrm{mag}]$ & $F_{\mathrm{X}}^{a}$ & Identification & Reference \\
\hline MP J0003-3556 & 0.0497 & 13.2 & 0.5206 & ABELL 2717 & Collins et al. 1995 \\
MP J0103-3225 & $\ldots$ & 19.0 & & IRAS F01009-3241 & Condon et al. 1995 \\
MP J1250-4026 & $\ldots$ & $\ldots$ & 0.000595 & 1RXP J125006-402 & Voges et al. 1999 \\
MP J1943-4030 & $\ldots$ & 17.3 & & ABELL 3646 & Abell et al. 1989 \\
MP J2313-4243 & 0.0564 & 14.6 & 2.024 & ABELL S1101 & Stocke et al. 1991 \\
MP J2357-3445 & 0.0490 & 13.7 & 2.27 & ABELL 4059 & Postman \& Lauer 1995 \\
\hline
\end{tabular}

${ }^{a} F_{\mathrm{X}}$ is the number of X-ray counts $\mathrm{s}^{-1}$ as listed in the cited catalogs. 


\section{Appendix B: Radio maps}

\section{B.1. VLA maps of the WN sample}

VLA maps of the WN sample. The contour scheme is a geometric progression in $\sqrt{2}$, which implies a factor 2 change in surface brightness every 2 contours. The first contour level, indicated above each plot, is at $3 \sigma_{\mathrm{rms}}$, where $\sigma_{\text {rms }}$ is the rms noise determined around the sources. The restoring beams are indicated in the lower left corner of the plots. Two maps are given for each source, one showing a $6^{\prime}$ field of view to show possible related components, and a smaller blow-up of the source to show its morphology. The open cross indicates the NVSS position. Sources identified on the POSS have been marked in the top right corner.

\section{B.2. FIRST maps of the WN sample}

FIRST maps of the WN sample. Contours are as in Sect. B.1.

\section{B.3. VLA maps of the TN sample}

VLA maps of the TN sample. Contours are as in Sect. B.1.

\section{B.4. FIRST maps of the TN sample}

FIRST maps of the TN sample. Contours are as in Sect. B.1.

\section{B.5. ATCA maps of the TN sample}

ATCA maps of the TN sample. Contours are as in Sect. B.1.

\section{B.6. ATCA maps of the MP sample}

ATCA maps of the MP sample. Contours are as in Sect. B.1.

\section{Appendix C: Radio spectra}

\section{C.1. Radio spectra for the WN sample}

Radio spectra of the WN sample using data from the literature. The two connected flux points indicate the spectral index used to select the source in the USS sample. Note the steeper spectra with higher frequency in most objects.

\section{C.2. Radio spectra for the TN sample}

Radio spectra of the TN sample using data from the literature. The two connected flux points indicate the spectral index used to select the source in the USS sample. Note the steeper spectra with higher frequency in most objects.

\section{C.3. Radio spectra for the MP sample}

Radio spectra of the MP sample using data from the literature and our ATCA observations (diamonds). The two connected flux points indicate the spectral index used to select the source in the USS sample. Note the steeper spectra with higher frequency in most objects.

\section{Appendix D: POSS finding charts}

\section{D.1. POSS finding charts for the WN sample}

POSS finding charts of the WN sample. The open cross indicates the NVSS position.

\section{D.2. POSS finding charts for the TN sample}

POSS finding charts of the TN sample. The open cross indicates the NVSS position.

\section{D.3. POSS finding charts for the MP sample}

POSS finding charts of the MP sample. The open cross indicates the MRC position.

\section{References}

Abell G.O., Corwin H.G. Jr., Olowin R.P., 1989, ApJS 70, 1 Andernach H., Verkhodanov O., Verkhodanova N., 2000, IAU Symp. 199 (in press) astro-ph/0001473

Bade N., et al., 1998, A\&AS 127, 145

Barthel P.D., 1989, ApJ 336, 606

Becker R.H., White R.L., Helfand D.J., 1995, ApJ 450, 559

Benn C.R., Rowan-Robinson M., McMahon R., Broadhurst T., Lawrence A., 1993, MNRAS 263, 98

Best P., Longair M., Röttgering H., 1998, MNRAS 295, 549

Blumenthal G., Miley G. 1979, A\&A 80, 13

Blundell K., Rawlings S., Eales S., Taylor G., Bradley A.D., 1998, MNRAS 295, 265

Blundell K., Rawlings S., Willott C., 1999, AJ 117, 677

Bremer M.N., Rengelink R., Saunders R., Röttgering H.J.A., Miley G.K., Snellen I.A.G., 1998, in Observational Cosmology with the New Radio Surveys, Bremer M.N., Jackson N., Pérez-Fournon I. (eds.). Dordrecht: Kluwer, p. 165 
Carilli C.L., Röttgering H.J.A., van Ojik R., Miley G.K., van Breugel W.J.M., 1997, ApJS 109, 1

Carilli C.L., Röttgering H.J.A., Miley G.K., Pentericci L.H., Harris D.E., 1999, in Proc. The most distant radio galaxies, Röttgering H.J.A., Best P.N., Lehnert M.D. (eds.). Amsterdam: KNAW, p. 123

Chambers K.C., Miley G.K., van Breugel W.J.M., Huang J.-S., 1996, ApJS 106, 215

Chambers K.C., Miley G.K., van Breugel W.J.M., Bremer M.A.R., Huang J.-S., Trentham N.A., 1996, ApJS 106, 247

Coleman P.H., Condon J.J., 1985, AJ 90, 8

Collins C.A., Guzzo L., Nichol R.C., Lumsden S.L., 1995, MNRAS 274, 1071

Condon J.J., Anderson E., Broderick J.J., 1995, AJ 109, 2318

Condon J., et al., 1998, AJ 115, 1693

Crawford C., et al., 1995, MNRAS 274, 75

De Breuck C., van Breugel W., Röttgering H., Miley G., 1998, in Observational Cosmology with the New Radio Surveys, Bremer M.N., Jackson N., Pérez-Fournon I. (eds.). Dordrecht: Kluwer, p. 185

De Breuck C., Brotherton M.S., Tran H.D., van Breugel W., Röttgering H., 1998, AJ 116, 13

De Breuck C., van Breugel W., Röttgering H., Miley G., Stern D., 2000 (in preparation)

de Ruiter H.R., Willis A.G., Arp H.C., 1977, A\&A 28, 211

Dey A., Spinrad H., Stern D., Graham J.R., Chaffee F.H., 1998, ApJ 498, L93

Dickinson M.E., in Proc. STScI May 1997 Symposium "The Hubble Deep Field", Livio M., Fall S.M., Madau P. (eds.). Cambridge: Cambridge Univ. Press (in press) astro-ph/9802064

Douglas J.N., Bash F.N., Bozyan F.A., Torrence G.W., Wolfe C., 1996, AJ 111, 1945

Dunlop J., Peacock J., Spinrad H., Stern D., Windhorst R.A., 1996, Nat 381, 581

Eales S., Rawlings S., Law-Green D., Cotter G., Lacy M., 1997, MNRAS 291, 593

Faranoff B.L., Riley J.M., 1974, MNRAS 167, 31

Ficarra A., Grueff G., Tomassetti G., 1985, A\&AS 59, 255

Frater R.H., Brooks J.W., Whiteoak J.B., 1992, JEEE, Australia 12 (2), 103

Giovannini G., Tordi M., Feretti L., 1999, New Astron. 4, 141

Gregg M.D., Becker R.H., White R.L., Helfand D.J., McMahon R.G., Hook I.M., 1996, AJ 112, 407

Gregory P.C., Condon J.J., 1991, ApJS 75, 1011

Griffith M., Wright A.E., 1993, AJ 105, 1666

Griffith M., Wright A.E., Burke B.F., Ekers R.D., 1994, ApJS 90, 179

Griffith M., Wright A.E., Burke B.F., Ekers R.D., 1995, ApJS 97, 347

Hales S.E.G., Baldwin J.E., Warner P.G., 1993, MNRAS 263, 25

Heckman T.M., O’Dea C.P., Baum S.A., Laurikainen E., 1994 ApJ 428, 65

Hook I.M., McMahon R.G., 1998, MNRAS 294, 7L

Hu E.M., McMahon R.G., Cowie L.L., 1999, ApJ 522, L9

Hughes D.H., Dunlop J.S., Rawlings S., 1997, MNRAS 289, 766

Ivison R., et al., 1998, ApJ 494, 211

Jarvis M., Rawlings S., Willot C., Blundell K., Eales S., Lacy M., 1999, in Proc. "The Hy-redshift universe" (in press) astro-ph/9908106
Kaplan D.L., Condon J.J., Arzoumanian Z., Cordes J.M., 1998, ApJS 119, 75

Komissarov S.S., Gubanov A.G., 1994, A\&A 285, 27

Krolik J.H., Chen W., 1991, AJ 102, 1659

Lacy M., Hill G., Kaiser M.-E., Rawlings S., 1993, MNRAS 263,707

Lacy M., et al., 1994, MNRAS 271, 504

Large M.I., Mills B.Y., Little A.G., Crawford D.F., Sutton J.M., 1981, MNRAS 194, 693

Laing R.A., Riley J., Longair M., 1983, MNRAS 204, 151

Lilly S.J., Longair M.S., 1984, MNRAS 211, 833

Lilly S.J., 1989, ApJ 340, 77

Lorimer D.R., Yates J.A., Lyne A.G., Gould D.M., 1995, MNRAS 273, 411

Machalski J., Ryś S., 1981, A\&A 99, 388

Martel A., et al., 1998, AJ 115, 1348

McCarthy P., 1993, ARA\&A 693

McCarthy P., Kapahi V.K., van Breugel W., Persson S.E., Atheya R.M., Subrahmanya C.R., 1996, ApJS 107, 19

McLure R., Dunlop J., 2000, MNRAS (in press) astro-ph/9908214

Napier P.J., Thompson A.R., Ekers R.D., 1983, Proc. IEEE 71,1295

Rawlings S., Lacy M., Blundell K., Eales S, Bunker A., Garrington S., 1996, Nat 383, 502

Odewahn S.C., Aldering G., 1996 (private communication to NED)

Oort M.J.A., Katgert P., Steeman F.W.M., Windhorst R.A., 1987, A\&A 179, 41

Oort M.J.A., 1988, A\&A 193, 5

Owen F.N., Condon J.J., Ledden J.E., 1983, AJ 88, 1

Owen F.N., Laing R.A., 1989, MNRAS 238, 357

Pedani M., Grueff G., 1999, A\&A 350, 368

Postman M., Lauer T.R., 1995, ApJ 440, 28

Pursimo T., et al., 1999, A\&AS 134, 50

Rawlings S., Eales S., Warren, 1990, MNRAS 243, 14P

Rawlings S., Blundell K.M., Lacy M., Willot C.J., 1998, in Observational Cosmology with the New Radio Surveys, Bremer M.N., Jackson N., Pérez-Fournon I. (eds.). Dordrecht: Kluwer, p. 171

Rees N., 1990, MNRAS 244, 233

Rengelink R., et al., 1997, A\&A 124, 259

Rengelink R., 1998, Ph.D. Thesis, Rijksuniversiteit Leiden

Röttgering H.J.A., Lacy M., Miley G.K., Chambers K.C., Saunders R., A\&AS 108, 79

Röttgering H.J.A., Miley G.K., Chambers K.C., Machetto F., A\&AS 114, 51

Röttgering H.J.A., van Ojik R., Miley G.K., Chambers K.C., van Breugel W.J.M., de Koff S., 1997, A\&A 326, 505

Shakhbazian R.K., 1973, Astrofizika 9, 495

Sheppard M.C., 1997, in ASP Conf. Ser. 125, Astronomical Data Analysis Software and Systems VI, Hunt G., Payne H.E. (eds.). San Francisco: Astron. Soc. of the Pacific, p. 77

Spinrad H., Djorgovski S., Marr J., Aguilar L., 1985, PASP 97, 932

Spinrad H., et al., 1997, ApJ 484, 581

Spinrad H., et al., 1998, AJ 116, 2617

Steidel C.C., Adelberger K.L., Giavalisco M., Dickinson M., Pettini M., 1999, ApJ 519, 1

Stocke J., et al., 1991, ApJS 76, 813

Tielens A.G.G.M., Miley G.K., Willis A.G., 1979, A\&AS 35, 153 
van Breugel W., Stanford S.A., Spinrad H., Stern D., Graham J.R., 1998, ApJ 502, 614

van Breugel W., De Breuck C., Röttgering H., Miley G., Stanford A., 1999, in "Looking Deep in the Southern Sky", Morganti R. (ed.). Sydney, p. 236

van Breugel W., De Breuck C., Stanford S.A., Stern D., Röttgering H., Miley G., 1999, ApJ 518, 61

Verkhodanov O.V., Trushkin S.A., Andernach H., Chernenkov V.N., 1997, in "Astronomical Data Analysis Software and Systems VI", Hunt G., Payne H.E. (eds.). ASP Conf. Ser. $125,322-325$

Vigotti M., Grueff G., Perley R., Clark B.G., Bridle A., 1989, AJ 98, 419

Voges W., et al., 1999, A\&A 349, 389

Waddington I., Windhorst R., Cohen S., Partridge R., Spinrad H., Stern D., 1999, ApJ 526, L77

Weymann R., et al., 1998, ApJ 505, L95
White N.E., Giommi P., Angelini L., 1994, IAU Circ. 6100, 1 White R.L., Becker R.H., Helfand D.J., Gregg M.D., 1997, ApJ 475,479

White R.L., et al., 2000, ApJS (in press) astro-ph/9912215

Wieringa M.H., Ph.D. Thesis, Rijksuniversiteit Leiden

Wieringa M.H., Katgert P., 1992, A\&AS 93, 399

Windhorst R.A., Miley G.K., Owen F.N., Kron R.G., Koo D.C., 1985, ApJ 289, 494

Witzel A., Schmidt J., Pauliny-Toth I.I.K., Nauber U., 1979, AJ 84, 942

Wright A.E., Griffith M.R., Burke B.F., Ekers R.D., 1994, ApJS 91, 111

Wright A.E., Griffith M.R., Troup E., Hunt A., Burke B.F., Ekers R.D., 1996, ApJS 103, 145

Zabuldoff A.I., Geller M.J., Huchra J.P., Vogeley M.S., 1993, AJ 106, 1273 